Rodolfo Oliveira

\title{
Finite Element Method Applied to Flow in Heterogeneous Porous \\ Media
}

\section{DISSERTAÇÃO DE MESTRADO \\ DEPARTMENTO DE ENGENHARIA MECÂNICA Programa de Pós-Graduação em Engenharia Mecânica}


Pontifícia Universidade Católica

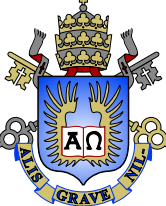

Rodolfo Oliveira

Finite Element Method Applied to Flow in Heterogeneous Porous Media

Thesis presented to the Programa de Pós-Graduação em Engenharia Mecânica of the Departamento de Engenharia Mecânica, PUC-Rio as partial fulfillment of the requirements for the degree of Mestre em Engenharia Mecânica.

Advisor: Prof. Márcio da Silveira Carvalho 


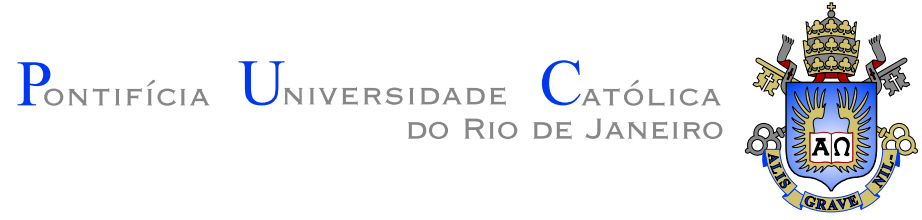

Rodolfo Oliveira

\section{Finite Element Method Applied to Flow in Heterogeneous Porous Media}

Thesis presented to the Programa de Pós-Graduação em Engenharia Mecânica of the Departamento de Engenharia Mecânica, PUC-Rio as partial fulfillment of the requirements for the degree of Mestre em Engenharia Mecânica.

Prof. Márcio da Silveira Carvalho

Advisor

Departmento de Engenharia Mecânica - PUC-Rio

Prof. Angela Ourivio Nieckele

Departamento de Engenharia Meânica - PUC-Rio

Prof. Juliana Vianna Valério

Universidade Federal do Rio de Janeiro - UFRJ

Prof. José Eugênio Leal

Coordinator of the Centro Técnico Científico da PUC-Rio 
All rights reserved.

\section{Rodolfo Oliveira}

Rodolfo Oliveira graduated from the Federal University of Espírito Santo in Petroleum Engineering. During his undergraduate course he was awarded the Society of Petroleum Engineering $1^{\text {st }}$ prize at the 2010 Latin American and Caribbean Undergraduate Student Paper Contest and the $3^{\text {rd }}$ prize at the 2011 International Undergraduate Student Paper Contest and during the elaboration of this work the 2012 Society of Petroleum Engineering Star Fellowship.

Ficha Catalográfica

Oliveira, Rodolfo

Finite Element Method Applied to Flow in Heterogeneous Porous Media / Rodolfo Oliveira; advisor: Márcio da Silveira Carvalho. - 2014.

90 f: il. ; $30 \mathrm{~cm}$

1. Dissertação (mestrado) - Pontifícia Universidade Católica do Rio de Janeiro, Departmento de Engenharia Mecânica, 2014.

Inclui bibliografia.

1. Engenharia mecânica - Teses. 2. Método dos elementos finitos. 3. Permeabilidade. 4. Equação de Brinkman. 5. Área representative elementar. 6. Vugos. I. Carvalho, Márcio da Silveira. II. Pontifícia Universidade Católica do Rio de Janeiro. Departmento de Engenharia Mecânica. III. Título. 


\section{Acknowledgments}

To Prof. Márcio da Silveira Carvalho and all professors of the Departamento de Engenharia Mecânica for the technical support.

To CNPq and PUC-Rio for the financial support.

To my parents and beloved wife for the unconditional support. 


\section{Abstract}

Oliveira, Rodolfo; Carvalho, Márcio da Silveira. Finite Element Method Applied to Flow in Heterogeneous Porous Media. Rio de Janeiro, 2014. 90p. Dissertação de Mestrado - Departmento de Engenharia Mecânica, PUC-Rio.

Carbonate rocks have unique attributes that distinguish them from siliciclastics and that require different methods of study to characterize their texture. Carbonates rocks are formed as a result of close interactions between biological and chemical depositional processes. The underlying diagenetic processes that form and alter these rocks contribute to a build-up of heterogeneities. Because of the high heterogeneity content measured properties (e.g. porosity and permeability) change with the scale of investigation and studies have struggle with a trade-off between significance of details and space representativeness. Extending a smaller scale to a larger requires scaling up procedures that preserves the essence of physical processes at one level to be summarized at the coarser level. Simplistic methods for scaling-up non-additive properties such as permeability generally do not honour the original heterogeneity present in complex systems. Therefore the dynamics of fluid flow in complex rocks demand more sophisticate methods and approaches. This study was focused in developing a methodology to evaluate the permeability as a scaling-up parameter for heterogeneous porous media. The permeability is back-calculated by emulating Darcy's experiment and solving the pore-scale ow using a Finite element formulation of Brinkman flow equation. The study was initially focused on parametric systems of periodic cells and later extended to two micro-tomography carbonate samples in which one has been selected for a spatial representativeness study. The parametric cells were used to evaluate the shape effects of channels and void spaces in an analogy to geological fractures and vugs as well as the permeability of the porous matrix. The micro-tomography carbonate samples consisted of a real case scenario in which, to a certain degree, could be observed a combination of the previously studied periodic cells. Finally a representativeness study was conducted segmenting the micro-tomography sample into sufficiently sub-samples that would be capable of reproducing the spatial heterogeneity of the sample.

\section{Keywords}

Finite element method; Permeability; Brinkman equation; Representative elementary area; Vugs; 


\section{Resumo}

Oliveira, Rodolfo; Carvalho, Márcio da Silveira. Método de Elementos Finitos Aplicados a Fluxo em Meios Porosos Heterogêneos. Rio de Janeiro, 2014. 90p. Dissertação de Mestrado - Departmento de Engenharia Mecânica, PUC-Rio.

Rochas carbonáticas possuem atributos únicos que as distinguem das siliciclasticas e necessitam de diferentes métodos de estudo para caracterizar sua textura. Rochas carbonáticas são resultado de interações entre processos deposicionais químicos e biológicos. Os processos diagenéticos que formam e alteram estas rochas contribuem para o acúmulo de heterogeneidades. Devido ao alto teor de heterogeneidade, as propriedades medidas (e.g. porosidade e permeabilidade) variam com a escala de investigação e estudos com o compromisso a significância dos detalhes e representação espacial. Ampliar uma escala menor para maior, exige procedimentos que preservem a essência dos processos físicos de um nível mais detalhada para um mais grosseiro. Métodos simplificados para dimensionar propriedades não aditivas em outras escalas, tal como permeabilidade, geralmente não honram a heterogeneidade presente em sistemas complexos. Com isso a dinâmica dos fluidos em rochas complexas exige abordagens e métodos mais sofisticados. Este estudo é focado em desenvolver uma metodologia de avaliação da permeabilidade como parâmetro de escala para meios porosos heterogêneos. A permeabilidade é retro calculada ao emular o experimento de Darcy e resolver o fluxo no meio poroso utilizando uma formulação de elementos finitos para equação de Brinkman. O estudo iniciou com foco em sistemas paramétricos de células periódicas e posteriormente a duas microtomogra fia de rochas carbonáticas, do qual uma foi selecionada para um estudo de representatividade espacial. As células periódicas foram utilizadas para avaliar os efeitos da formação de canais e espaços vazios, em analogia a fraturas e vugos, assim como os efeitos da permeabilidade da matriz porosa. As amostras carbonáticas consistem de casos nos quais é possível observar a presença de uma combinação dos fenômenos estudadas. Por fim um estudo de representatividade foi conduzido segmentando uma amostra de micro-tomografia em suficientes sub-amostras que capazes de reproduzir a heterogeneidade espacial da amostra original.

\section{Palavras-chave}

Método dos elementos finitos; Permeabilidade; Equação de Brinkman; Área representative elementar; Vugos; 


\section{Contents}

$\begin{array}{lr}\text { List } \text { of Figures } & 9\end{array}$

$\begin{array}{ll}\text { List of Tables } & 12\end{array}$

1 Introduction $\quad 14$

1.1 Carbonate Rocks 14

$\begin{array}{lll}1.2 & \text { Scales of Investigation } & 15\end{array}$

1.3 Porous Media Flow 16

$\begin{array}{lll}1.4 & \text { Objectives } & 17\end{array}$

$\begin{array}{ll}1.5 & \text { Division of Chapters } \\ \end{array}$

2 Governing Equations $\quad 20$

2.1 The Continuum Concept 20

2.1.1 The Fluid as a Continuum 21

2.1.2 The Rock as a Continuum 22

2.2 Mass Conservation 23

2.3 Linear Momentum Conservation 24

2.3.1 Incompressible Navier-Stokes Equation 24

2.3.2 Stokes flow 25

2.3.3 Darcy's Law 25

2.3.4 Brinkman's Equation 25

3 Numerical Formulation $\quad 27$

3.1 The Finite Element Method 27

3.2 The FEniCS Project and DOLFIN Library 27

$\begin{array}{ll}3.3 \text { Poisson's Equation } & 29\end{array}$

3.4 Darcy's Flow Equation 33

3.4.1 Darcy's Variational Formulation 33

3.4.2 Darcy's Finite Element Implementation 33

$\begin{array}{ll}\text { 3.4.3 Darcy's Example } & 35\end{array}$

3.5 Stokes' Flow Equation 36

3.5.1 Stokes' Variational Formulation 36

3.5.2 Stokes' Finite Element Implementation 37

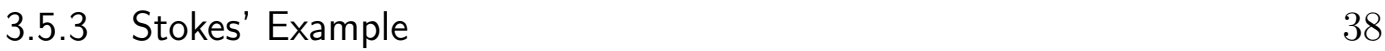

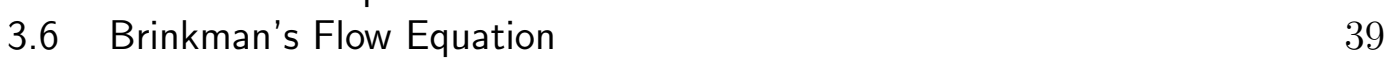

3.6.1 Brinkman's Variational Formulation 39

3.6.2 Brinkman's Finite Element Implementation 40

3.6.3 Brinkman's Example 41

4 Results $\quad 44$

4.1 A Layered Medium $\quad 45$

4.1.1 The Effect of the Fracture Aperture 45

4.1.2 The Effect of the Matrix Permeability 46

4.2 A Porous Medium with Vugs 48 
4.2.1 The Effect of the Size of the Vug 48

4.2.2 The Effect of the Vug Shape 50

4.3 A Porous Medium System 53

4.4 Core Samples 58

4.4.1 An Austin Chalk Sample $\quad 58$

4.4.2 A Coquinas Analogue Sample 60

4.5 Representative Elementary Study 63

5 Discussions and Conclusions $\quad 68$

$\begin{array}{lll}5.1 & \text { Periodic Cells } & 68\end{array}$

5.2 Austin Chalk and Coquinas analogue samples 69

$\begin{array}{ll}5.3 \text { Representative elementary study } & 69\end{array}$

$\begin{array}{ll}\text { Bibliography } & \mathbf{7 0}\end{array}$

$\begin{array}{lr}\text { Appendices } & 75\end{array}$

$\begin{array}{lll}\text { Appendix A } & \text { FEniCS Algorithms } & \mathbf{7 6}\end{array}$

$\begin{array}{ll}\text { A.1 Poisson Equation } & 76\end{array}$

$\begin{array}{lll}\text { A.2 Darcy Equation } & 79\end{array}$

A.3 Stokes Equation $\quad 83$

$\begin{array}{lll}\text { A.4 Brinkman Equation } & 87\end{array}$ 


\section{List of Figures}

1.1 Conceptual sketch of different scales of investigation. From left to right a representation of pore, core and field scales figs. 1.1(a) to $1.1(\mathrm{c})$.

2.1 Idealization of a Representative Elementary Volume (REV) for a generic fluid.

2.2 Idealization of a Representative Elementary Volume (REV) for a generic rock.

2.3 Mass balance and stresses for a differential elementary volume shown only in the $x$-direction.

3.1 Overview structure of the FEniCS Project, from [35].

3.2 Solution for Poisson equation described at eq. (A-1) and generated using the FEniCS package [35].

3.3 Porous medium with different permeability. The domain size is $l$ and is subdivided in two domains each with height $l_{1 / 2}=l / 2$. The sub-domain $\Omega_{D 1}$ has permeability $k=10 \mathrm{mD}$ and $\Omega_{D 2}$ permeability $k=100 \mathrm{mD}$.

3.4 Darcy's flow equation solved for a porous medium of permeability $k$ and fluid viscosity $\mu$.

3.5 Parallel plates domain. The domain size is $l$ and is bounded by two walls, one on top and the other on the bottom of the domain.

3.6 Stokes' flow equation solved for parallel plates and fluid viscosity $\mu$. 39

3.7 Porous medium with circular inclusion. The domain size is $l$ and is subdivided in two domains. The sub-domain $\Omega_{D}$ is porous domain and has permeability of $k=100 \mathrm{mD}$ and $\Omega_{S}$ is a free-fluid domain.

3.8 Brinkman's flow equation solved for an isotropic medium of permeability $k$ and fluid viscosity $\mu$ with the presence of a circular free-fluid region.

4.1 Periodic cell showing a vug of arbitrary shape. The domain, $\Omega$, is composed of two sub-domains, $\Omega_{D}$, with the subscript $D$ corresponding to the Darcy sub-domain, and $\Omega_{S}$, with the subscript $S$ corresponding to the Stokes sub-domain, the last corresponding to the vug region. The boundaries, $\Gamma$, is split into $\Gamma_{1}, \Gamma_{2}, \Gamma_{3}$ and $\Gamma_{4}$, respectively corresponding to the left, top, right and bottom of the domain

4.2 A periodic cell for the layered medium of size $l \times l$ and conduit aperture $a$. The $\Omega_{D}$ and $\Omega_{S}$ respectively stands for Darcy and Stokes sub-domains. The discrete mesh is refined towards the subdomains interface and finer inside $\Omega_{S}$ sub-domain. 
4.3 Comparison between the estimated permeability for the periodic layered cell, the blue points, and the fracture permeability, the continuous blue line. The $x$-axis shows the fracture aperture $a / l$ and the $y$-axis shows the estimated absolute permeability.

4.4 Velocity profile for a line crossing $[0.5,0.5] \times[0.0,1.0]$ over the the layered cell $4.2(\mathrm{a})$ with conduit aperture of $a / l=0.20$ highlighting the interface between $\Omega_{D}$ and $\Omega_{S}$. The solid black line shows the estimated velocity profile and the blue tick line the profile for a parallel plates flow.

4.5 Four different vugs shape with periodic cell size $l$. The circle radius at fig. 4.5(a) is defined as $r$. The square side size at fig. 4.5(b) is defined as $l$. The radius of the circumscribed hexagon at fig. 4.5(c) is $l_{h}$. The cross at fig. 4.5(d) extension is $l_{e}$ and the width $a$. All cells respects the ratio $\Omega_{D} / \Omega=1 / 4$. The $\Omega_{D}$ and $\Omega_{S}$ respectively stands for Darcy and Stokes sub-domains. The figures are out of scale and are only used for illustrative matters.

4.6 Effect of varying radius at a circle-shaped vug. The $x$-axis shows the length $r / l$ and the $y$-axis shows the estimated absolute permeability for the periodic cell.

4.7 Effect of different vug shape. The $x$-axis shows the matrix permeability and the $y$-axis shows the estimated absolute permeability for the periodic cell.

4.8 Pressure and velocity field for periodic cells described in fig. 4.5. The sub-domains area of the periodic cells is constant and set as $A_{\Omega_{D}} / A_{\Omega}=1 / 4$.

4.9 Porous medium system with interconnected fractures, isolated vugs and fracture-connected vugs. The periodic cell size is $l$, the $\Omega_{D}$ and $\Omega_{S}$ respectively stands for Darcy and Stokes sub-domains. System of connected fractures with $l / l_{f}=0.5$ and fracture aperture $a / l=0.01$. System of isolated vugs with $r_{1} / l=0.25, r_{2} / l=0.10$, $a / l=0.25$ and $b / l=0.50$. The system of connected vugs is simply the superposition of the isolated vugs fig. 4.9(b) and connected fractures system fig. 4.9(a).

4.10 Velocity magnitude and pressure distribution for the system of isolated inclusions described at 4.9(b).

4.11 Velocity magnitude and pressure distribution for the system of connected fractures described at fig. 4.9(a).

4.12 Velocity magnitude and pressure distribution for the system of connected inclusions described at 4.9(c).

4.13 Micro-tomography ( $\mu \mathrm{CT})$ slice from an Austin Chalk sample. The red square in fig. 4.13(a) delimitates the studied region and its displayed at fig. 4.13(b). Figure 4.13(c) shows the numerical mesh in red overlapping regions with discrete properties.

4.14 Velocity magnitude and pressure distribution for the microtomography slice of Austin Chalk sample shown at 4.13. 
4.15 Micro-tomography ( $\mu \mathrm{CT}$ ) slice from a Coquinas analogue sample. The red square in fig. 4.15(a) delimitates the studied region and its displayed at fig. 4.15(b). Figure 4.15(c) shows the numerical mesh in red overlapping regions with discrete properties.

4.16 Velocity magnitude and pressure distribution for the microtomography slice of Coquinas analogue sample shown at 4.15.

4.17 Representative Elementary Area (REA) size partitioning and number of sub-samples.

4.18 Representative Elementary Area (REA) results for the estimated permeability in the $x$-direction. The color of the points gets darker as more points occupies the same region. Sub-samples being displayed at the top exemplifies the channels that communicates opposite faces of the REA.

4.19 Representative Elementary Area (REA) results for the estimated permeability in the $y$-direction. The color of the points gets darker as more points occupies the same region. Sub-samples being displayed at the top exemplifies the channels that communicates opposite faces of the REA.

4.20 Representative Elementary Area (REA) results for the estimated permeability in the $x$-direction with sub-samples with communicated opposite faces filtered. The color of the points gets darker as more points occupies the same region. The darker blue shade filling corresponds to the first standard deviation from the average and the lighter blue shade filling the second standard deviation from the average.

4.21 Representative Elementary Area (REA) results for the estimated permeability in the $y$-direction with sub-samples with communicated opposite faces filtered. The color of the points gets darker as more points occupies the same region. The darker blue shade filling corresponds to the first standard deviation from the average and the lighter blue shade filling the second standard deviation from the average.

4.22 Representative Elementary Area (REA) minimum size estimation for $x$-direction, using the first standard deviation $\sigma$, blue dots, and the laboratory measured permeability of the sample, blue line. The estimated REA minimun size was $l=1.27 \mathrm{~cm}$.

4.23 Representative Elementary Area (REA) minimum size estimation for $y$-direction, using the first standard deviation $\sigma$, blue dots, and the laboratory measured permeability of the sample, blue line. The estimated REA minimun size was $l=1.59 \mathrm{~cm}$. 


\section{List of Tables}

1.1 Comparison of terrigenous sandstones and carbonate reservoir characteristics [1, 2].

4.1 Effect of the varying aperture $a / l$ for the estimated permeability of the periodic layered domain shown at fig. 4.2(a) and the fracture permeability defined by eq. (4-1).

4.2 Effect of the varying matrix permeability $k_{\text {matrix }}$ over $\Omega_{D}$ subdomain for the periodic layered domain shown at fig. 4.2(a). The table contains the average velocity for the layered domain $u_{\text {avg }}$ and the average velocity for a parallel plates flow $u_{\text {plates }}$ using eq. (4-2).

4.3 Permeability results for different radius of a circular-shaped vug with medium permeability fixed at $k_{\text {matrix }}=10 \mathrm{mD} \quad 50$

4.4 Effect of vug shape for varying matrix permability $k_{\text {matrix }}$. 51

4.5 Estimated permeability tensor for the isolated vugs, connected fractures and fracture-connected vugs system.

4.6 Core plug geometry details and routine core analysis results for the Austin Chalk sample.

4.7 Estimated permeability tensor for the Austin Chalk analogue sample. 59

4.8 Core plug geometry details and routine core analysis results for the Coquinas sample.

4.9 Estimated permeability tensor for the Coquinas analogue sample.

4.10 Representative Elementary Area (REA) length sizes and number of selected sub-samples.

4.11 Representative Elementary Area (REA) statistics for the estimated permeability in the $x$ - and $y$-direction. 
Essentially, all models are wrong, but some are useful.

George E. P. Box,

Empirical Model-Building and Response Surfaces. 


\section{Introduction}

\section{1}

\section{Carbonate Rocks}

Carbonate rocks have unique attributes that distinguish them from siliciclastics and that require different methods of study [1]. They are formed within the depositional basin by biological, chemical, and/or detrital processes.

They are largely made up of skeletal remains and other biological constituents that include fecal pellets, lime mud, and microbial mediated cements and lime mud. Chemical constituents are common in carbonates but are absent in most siliciclastics [2].

Table 1.1 shows a side-by-side comparison of terrigenous sandstones and carbonate reservoir characteristics. It serves only to illustrate the main differences and complexities that arises from both reservoir types.

Table 1.1: Comparison of terrigenous sandstones and carbonate reservoir characteristics $[1,2]$.

\begin{tabular}{|c|c|c|}
\hline Characteristic & Terrigenous Sandstones & Carbonates \\
\hline Primary porosity & Low & High \\
\hline Type of primary porosity & $\begin{array}{l}\text { Almost exclusively inter- } \\
\text { particle }\end{array}$ & $\begin{array}{l}\text { Inter-particle, intra- } \\
\text { particle, inter-crystalline, } \\
\text { moldic, vuggy, cavernous, } \\
\text { fenestral, or "constructed } \\
\text { void" }\end{array}$ \\
\hline Type of ultimate porosity & $\begin{array}{l}\text { Almost exclusively primary } \\
\text { inter-particle }\end{array}$ & $\begin{array}{l}\text { Highly variable owing to } \\
\text { different origins or pore } \\
\text { types }\end{array}$ \\
\hline Typical pore size & $\begin{array}{l}\text { Diameter and throat sizes } \\
\text { related to depositional tex- } \\
\text { ture }\end{array}$ & $\begin{array}{l}\text { Diameter and throat size } \\
\text { may not be related to de- } \\
\text { positional texture }\end{array}$ \\
\hline Typical pore shape & Varies with particle shape & $\begin{array}{l}\text { From strongly related to } \\
\text { totally unrelated to particle } \\
\text { shape }\end{array}$ \\
\hline $\begin{array}{l}\text { Uniformity of pore size and } \\
\text { shape distribution }\end{array}$ & $\begin{array}{l}\text { Relatively uniform in homo- } \\
\text { geneous sand bodies }\end{array}$ & $\begin{array}{l}\text { Fairly uniform to extremely } \\
\text { heterogeneous }\end{array}$ \\
\hline
\end{tabular}

Carbonate rock porosities are expected to vary significantly when compared to sandstones and its permeability varies accordingly. The underlying 
processes that form and alter these rocks contributes to the heterogeneity build-up. Two of the most usual classification methods are defined from Folk [3] and Dunham [4]. Folk [3] classifies carbonate rocks according to whether they have a micrite or a sparite matrix. Dunham [4] classifies carbonate rocks according to whether they are grain- or matrix-supported and depending on the dominant type of grain and matrix. Fully understanding of carbonate rocks requires clear descriptions in all scales, from pore- to field-scale

\section{2}

\section{Scales of Investigation}

One of the most important goals of modeling is to reduce the risk associated with making decisions in an environment where knowledge is limited. The validity of data used in the decision-making process depends on the measurement technique used to obtain the data and appropriate scale of applicability of the technique. Data validity provides information about risk. The integration of scale-dependent data into a cohesive reservoir description can reduce the risk of decision-making [5].

Figure 1.1 illustrates the information contained in three different conceptual scales of investigation: pore-scale, core-scale and field-scale. At the pore-scale is generally possible to identify mineral contacts and pore shape. The core-scale illustrates the pores connectivity and fluid distribution. Finally the field-scale contains the depositional basins and the fracture distribution. The measured properties change with scale of investigation and studies may struggle with a trade-off between significance of details and scale representativeness. Dominant processes and governing equations may vary with scales.

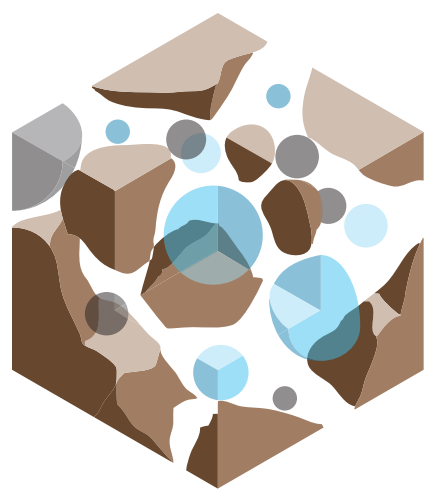

1.1(a): Pore-scale.

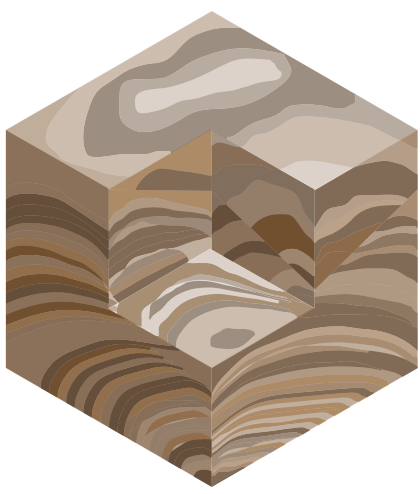

1.1(b): Core-scale.

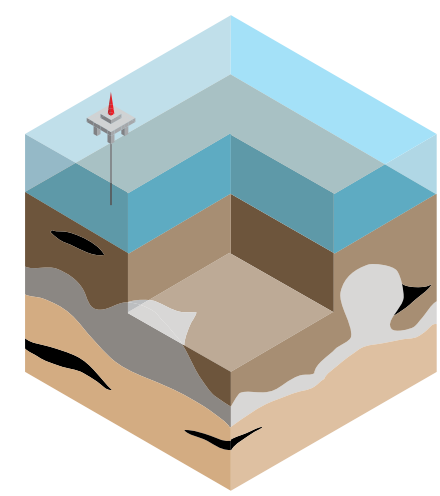

1.1(c): Field-scale.

Figure 1.1: Conceptual sketch of different scales of investigation. From left to right a representation of pore, core and field scales figs. 1.1(a) to 1.1(c).

Extending from one scale to a larger one requires scaling up procedures that 
preserve the essence of the physical processes at one level to be summarized at the coarser level.

\section{3}

\section{Porous Media Flow}

Some properties, such as porosity, can be summed or averaged in order to obtain representative values of given regions. When dealing with permeability other factors need to be included, i.e. the pores connectivity. Exact solutions exist for simple geometries, such as weighted- and harmonic-average permeability calculations [6]:

$$
\begin{aligned}
& k_{\text {avg }}=\frac{k_{1} \cdot h_{1}+\cdots+k_{n} \cdot h_{n}}{H}=\frac{\sum_{i=1}^{n} k_{i} \cdot h_{i}}{\sum_{i=1}^{n} h_{i}} \\
& k_{\text {avg }}=\frac{L}{l_{1} / k_{1}+\cdots+l_{n} / k_{n}}=\frac{\sum_{i=1}^{n} l_{i}}{\sum_{i=1}^{n} l_{i} / k_{i}}
\end{aligned}
$$

Equation (1-1a) is known as weighted-average permeability and averages a given permeability $k_{i}$ accordingly to the layer height $h_{i}$ in a medium with known total height $H$. Equation (1-1b) is known as harmonic-average permeability and averages the reciprocal permeability $k_{i}$ accordingly to the layer length $l_{i}$ in a medium with known total length $L$. The weighted-average permeability is used for simplistic cases when the flow direction is parallel to the bedding of a layered medium and the harmonic-average permeability when the flow direction is perpendicular to the bedding of a layered medium.

Although useful, in most cases eqs. (1-1a) and (1-1b) do not honor the original heterogeneity present in complex systems. The dynamics of fluid flow in complex rocks demand more sophisticated methods and approaches [7].

Among most methods, the Lattice Boltzmann Method (LBM) is a fluid simulation method that solves the discrete Boltzmann equation with a collision model on a grid, or lattice [8] and is commonly used for fluid flow simulations. It can be extended to represent fluid particles of two or more phases, however this significantly increase the computational cost of the simulations [9]. The method is widely applied to average macroscopic behavior of properties on rock samples. Ferréol and Rothman [10] applied it to estimate the permeability of a Fontainebleau sandstone sample.

Another widely used method to estimate porous media properties comes 
from network models. The first network model was proposed by Fatt [11] with an analogy between flow in porous media and electrical resistor networks. The technique significantly evolved in subsequent years and now accommodates several geometry corrections and physical phenomena, e.g. different pore shapes and wettability [12].

Density functional modeling develops a general formulation based on the entropy, or Helmholtz energy, as a functional that is dependent upon chemical component densities [13]. Koroteev et al. [14] reproduced snap-offs, capillary de-saturation and relative permeability phenomena that agrees with experiments for a poorly consolidated sandstone.

A more traditional approach involves numerically solving Navier-Stokes or Stokes equations in the domain defined by the pores. Those equations are used to describe the flow in micro- or pore-scale connected spaces. Rock pores and grains can be imaged using micro-tomography data.

Modeling flow properties at micro-scale generally would be considered a first step from which the spatial distribution of properties can be addressed. Scaling-up procedures are necessary in order to use the estimated properties on a coarser scale, such as macro- or reservoir-scale. Scaling-up techniques require the combined use of microscopic and macroscopic equations [15].

The usage of Darcy-Stokes coupled equations demands explicitly modeling the interfaces between the fluid and porous regions. ARbogast and Gomez [16] showed the Darcy-Stokes coupling using the Beavers-Joseph-Saffman (BJS) interfacial boundary condition. The problem was modeled using the finite element method and a multi-grid solver for the micro and macro system of equations.

A similar approach is provided by the Stokes-Brinkman or simply Brinkman equation, but without the need of BJS interfacial boundary conditions. The Brinkman equation can be reduced to either Stokes or Darcy equations with the appropriate choice of parameters, avoiding the need to explicit choose the formulation for the interfacial conditions [17]. Popov et al. [18] showed the validity of the Stokes-Brinkman equation as a fine-scale model for flow in vuggy, fractured karst reservoirs and compared the scaled up results with a coarse Darcy model.

\section{4}

\section{Objectives}

This study was focused on developing a methodology to evaluate the permeability as a scaling-up parameter for heterogeneous porous media. The permeability is back-calculated by numerically emulating Darcy's experiment 
and solving the pore-scale flow using a finite element formulation of Brinkman equation. The study was initially focused on parametric systems of periodic cells and later extended to two micro-tomography carbonate samples in which one has been selected for a spatial representativeness study. The parametric cells were used to evaluate the shape effects of channels and void spaces in an analogy to geological fractures and vugs, and of the permeability of the surrounding porous matrix. The micro-tomography carbonate samples consisted of a real case scenario in which, to a certain degree, could be observed a combination of the previously studied periodic cells. Finally a representativeness study was conducted segmenting the micro-tomography sample into enough sub-samples that would be capable of reproducing the spatial heterogeneity.

\section{5}

\section{Division of Chapters}

This dissertation is divided into five chapters that are briefly described next:

\section{Chapter 1 : Introduction}

Chapter 1 has just been presented and gives a short introduction on the objectives of this dissertation.

\section{Chapter 2 : Governing Equations}

Chapter 2 shows a short derivation on the continuum and representative elementary concepts. It also describes the main equations used in the dissertation as well as its fundamentals.

\section{Chapter 3 : Numerical Formulation}

Chapter 3 presents the variational and finite element formulation of the set of previously described equations. The computational implementation is briefly described using the Poisson equation with simple boundary conditions as an example in order to make a short introduction to the finite element package of choice, the FEniCS package.

\section{Chapter 4 : Results}

Chapter 4 starts with a permeability parametric study of fractures and vugs inserted in a periodic cell. Next two carbonate micro-tomography samples are used to obtain scale-up values of permeability and later one sample is used for a spatial representativeness study. 


\section{Chapter 5 : Discussions and Conclusions}

Chapter 5 summarizes the dissertation with the conclusions of the present investigation. 


\section{2 \\ Governing Equations}

\section{1}

\section{The Continuum Concept}

A general property can be defined differently accordingly to the length scale of investigation. The notion of a Representative Elementary Volume (REV) is of critical importance in order to derive effective properties from the constitutive laws and spatial distribution of their components.

The REV is usually regarded as a volume of a particular heterogeneous material that is sufficiently large to be statistically representative of the composite and yet small enough to be considered as a volume element of continuum mechanics. Traditionally it should include a sampling of all microstructural heterogeneities that occur in the composite material [19]. The Representative Elementary Area (REA) can be defined in a similar way, but evidently for an area instead of volume of investigation.

Drugan and Willis [20] defined the REV as the smallest material volume element of the composite for which the usual spatially constant (overall modulus) macroscopic constitutive representation is a sufficiently accurate model to represent mean constitutive response.

Kanit et al. [21] proposed a more quantitative definition of the REV, which is based on statistical arguments. The REV must ensure a given accuracy of the estimated property obtained by spatial averaging in a given domain. Alternatively, the use of smaller volumes must be compensated by averaging over several realizations of the microstructure to get the same accuracy, provided no bias is introduced in the estimation by some edge effects generated by the boundary conditions.

Nordahl and Ringrose [22] used numerical realizations of tidal deposits to determine the size of the REV accordingly to lithology content. A set of twelve models each with ten realizations were created to represent lithofacies deposited with a varied combination of sandstone and mudstone content. The estimated REV was found to vary as a function of lithofacies type and different scale up permeability. 
Standard local models are generally sensitive to material volume elements considerably larger than the microscopic scale of the material. However, when the macroscopic, or averaged, property fields do not vary slowly with respect to REV size, the standard local models break down, and more accurate macroscopic constitutive equations are required.

\subsection{1}

\section{The Fluid as a Continuum}

Fluids, as any other substance, are aggregation of molecules that are separated in a distance much larger than the molecules diameter. These molecules are not fixed in a lattice but move freely relative to each other. For a continuum or macroscopic approach to be valid, the size of the system must be larger than the mean free path of the molecules.

The density, as calculated from the molecular mass $\delta m$ within a given volume $\delta V$, is plotted versus the size of the fluid REV fig. 2.1. There should be a limiting volume $\delta V^{*}$ below which molecular variation prevents a proper measurement of density. The density of a given fluid is best defined as:

$$
\rho=\lim _{\delta V \rightarrow \delta V^{*}} \frac{\delta m}{\delta V}
$$

The limiting volume $\delta V^{*}$ is about $10^{-9} \mathrm{~mm}^{3}$ for all liquids and for gases at atmospheric pressure and ambient temperature [23]. At that volume and conditions the density of water is $998.2 \mathrm{~kg} / \mathrm{m}^{3}$ [24].
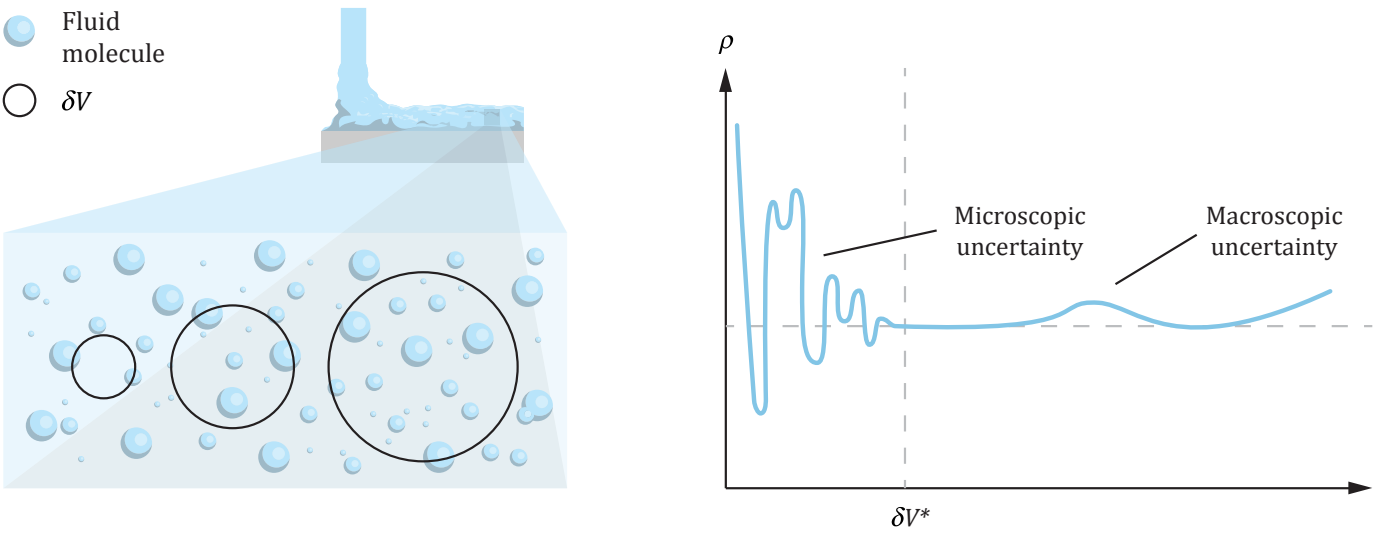

Figure 2.1: Idealization of a Representative Elementary Volume (REV) for a generic fluid.

The petroleum industry usually uses a different standard to classify oil density. The API gravity, or degree API, was developed by the American Petroleum Institute (API) for measuring the relative density of petroleum liquids. The scale is graded in degrees and the greater the density of petroleum 
liquid, the lower the API gravity [6]. The API gravity is calculated using the following relation:

$$
{ }^{\circ} A P I=\frac{141.5}{S G}-131.5
$$

with $S G$ being the petroleum specific gravity in relation to water at $60^{\circ} \mathrm{F}$.

Additionally the viscosity is another important property that characterizes fluids. A class of fluids known as Newtonian fluids have their velocity proportional to the imposed shear stress [25]. The constant of proportionality is the viscosity coefficient $\mu$ :

$$
\tau=\mu \frac{d u}{d y}
$$

To illustrate, water viscosity at atmospheric pressure and ambient temperature is $1.00210^{-3} \mathrm{~Pa} \cdot \mathrm{s}$ [24] whereas for the Athabasca bitumen 640.0 Pa.s and the Arabian light oil $14.010^{-3} \mathrm{~Pa} \cdot \mathrm{s}$ [26].

Density and viscosity are closely related but not in a straight forward relation. The most intensive control lies on the pressure and temperature changes, however there is a significant control with the composition which is likely to be related to the reservoir compartments [27].

\subsection{2}

\section{The Rock as a Continuum}

A REV of a rock is stated in a similar approach as for a fluid. The porosity, as calculated from the volume of pores $\delta V_{p}$ within the total volume $\delta V_{t}$ of a rock, is plotted versus the total volume of the REV (fig. 2.2). There should be a limiting volume $\delta V_{t}^{*}$ below which the microstructure heterogeneity prevents a proper measurement of the porosity. The porosity of a given rock is best defined as:

$$
\phi=\lim _{\delta V_{t} \rightarrow \delta V_{t}^{*}} \frac{\delta V_{p}}{\delta V_{t}}
$$

The porosity for sandstones can range from 0.04 to 0.30 [28]. The amount of interconnected pores defines the effective porosity, $\phi_{e}$, of the medium and the isolated porosity, the isolated pores.

A rock that presents interconnected pores can be characterized in terms of a macroscopic quantity, the permeability $k$. The permeability measures the rock's ability to transmit a given fluid and was first described by Darcy [29]. The Darcy unit is equivalent to $9.869 \times 10^{-13} \mathrm{~m}^{2}$ and 1 Darcy refers to the flow of $1 \mathrm{~cm}^{3} / \mathrm{s}$ of a fluid with $1 \mathrm{cP}$ of viscosity under a pressure gradient of $1 \mathrm{~atm} / \mathrm{cm}$ acting in an area of $1 \mathrm{~cm}^{2}$. To illustrate, the sandstones from the Gulfaks Cook formation have mean permeability of $500 \mathrm{mD}$ and reaches up to $5000 \mathrm{mD}[30]$. 

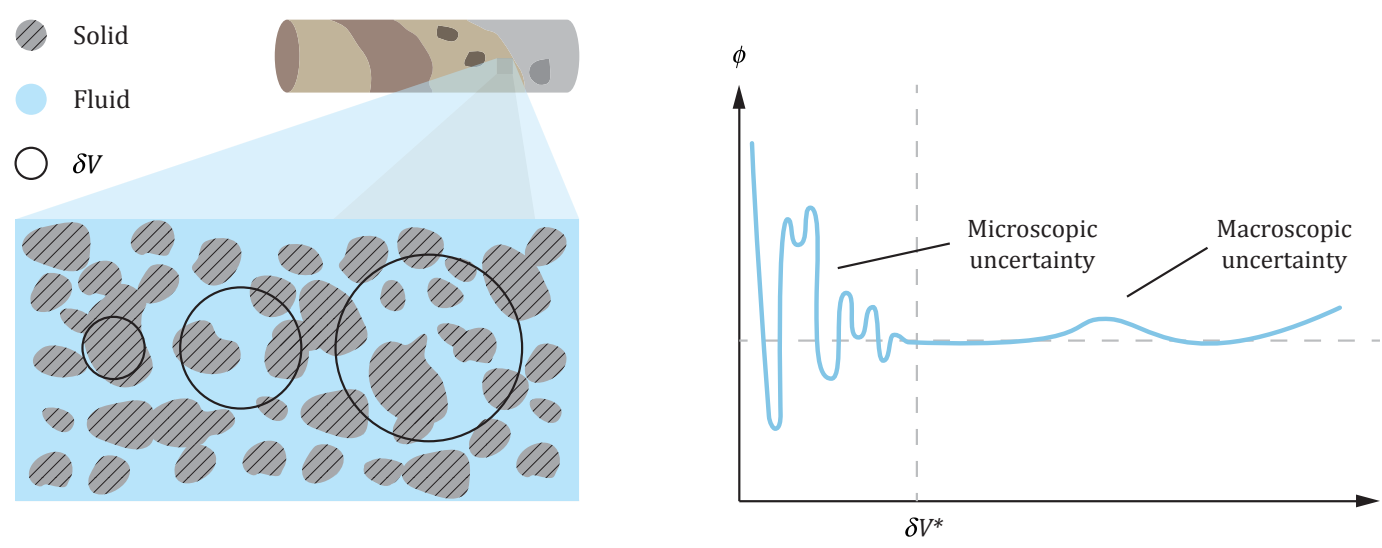

Figure 2.2: Idealization of a Representative Elementary Volume (REV) for a generic rock.

\section{2}

\section{Mass Conservation}

Consider an infinitesimal volume $\delta V$ fixed in space and bounded by a surface $\delta S$, whose sides $\delta x, \delta y$ and $\delta z$ are respectively parallel to the $x$-, $y$ and $z$-axis, as in fig. 2.3(a). Mass flow occurs from all six faces of the volume. Examining the mass flow in the $x$-direction, the inlet flux is $\rho u \delta y \delta z$ and the outlet flux $\left[\rho u+\frac{\partial}{\partial x}(\rho u) \delta x\right] \delta y \delta z$.

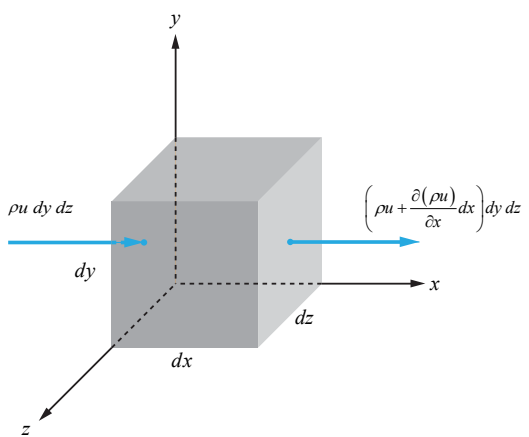

2.3(a): Mass balance.

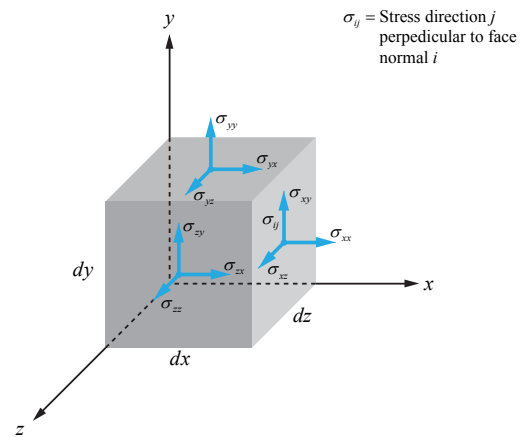

2.3(b): Stresses.

Figure 2.3: Mass balance and stresses for a differential elementary volume shown only in the $x$-direction.

Considering the rate of change of mass inside the infinitesimal volume and using vector notation, the equation of continuity becomes

$$
\frac{\partial \rho}{\partial t}+\nabla \cdot(\rho \mathbf{u})=0 \%
$$

where $\rho$ is the fluid density and $\mathbf{u}$ is the fluid's vector velocity. For an incompressible fluid, $\rho$ is constant in time and space and the continuity equation reduces to:

$$
\nabla \cdot \mathbf{u}=0 \%
$$




\section{3}

\section{Linear Momentum Conservation}

Using the infinitesimal volume $\delta V$ defined in fig. 2.3(b) and as analogy for the mass conservation, the linear momentum flux occur on all six faces of the volume. Examining the linear momentum flux in the $x$-direction, the inlet flux is $\rho u \mathbf{u} \delta y \delta z$ and the outlet flux $\left[\rho u \mathbf{u}+\frac{\partial}{\partial x}(\rho u \mathbf{u}) \delta x\right] \delta y \delta z$. This extends for all other directions and when combined to the continuity eq. (2-6) holds the relation to the net force acting on the infinitesimal element, or in other words:

$$
=\rho\left[\frac{\partial \mathbf{u}}{\partial t}+\mathbf{u} \cdot \nabla \mathbf{u}\right] \delta x \delta y \delta z
$$

The forces acting on the infinitesimal element are the surface forces and body forces. Considering gravity acting in the $z$-direction as the only body force and the surface forces only to stresses on the sides of the volume. Displaying the $x$-direction, fig. 2.3(b) shows the net force given by:

$$
\left[\frac{\partial}{\partial x} \sigma_{x x}+\frac{\partial}{\partial y} \sigma_{y x}+\frac{\partial}{\partial z} \sigma_{z x}\right] d x d y d z
$$

If we consider the stress normal to the $x$ face of the infinitesimal volume, $\sigma_{x x}$, due to the pressure and viscous stress, $-p+\left[\frac{\partial}{\partial x} \tau_{x x}\right]$, and the tangential stresses, $\sigma_{y x}$ and $\sigma_{z x}$ due to viscous stresses only, the net force equation in the $x$-direction, eq. (2-8) rewrites as:

$$
\left[-\frac{\partial}{\partial x} p+\frac{\partial}{\partial x} \tau_{x x}+\frac{\partial}{\partial y} \tau_{y x}+\frac{\partial}{\partial z} \tau_{z x}\right] d x d y d z
$$

In a similar manner for the $y$ - and $z$-direction, and using vector notation, the momentum equation for the infinitesimal volume becomes:

$$
\rho \mathbf{g}-\nabla p+\nabla \cdot \boldsymbol{\tau}=\rho\left[\frac{\partial \mathbf{u}}{\partial t}+\mathbf{u} \cdot \nabla \mathbf{u}\right]
$$

Some special cases of the linear momentum equation for an infinitesimal volume eq. (2-10) that will have importance on the present work are to be shown next.

\subsection{1}

\section{Incompressible Navier-Stokes Equation}

A special case of the linear momentum equation, eq. (2-10), regards the assumption of an incompressible Newtonian fluid flow, whose stresses are proportional to the rate of strain and viscosity:

$$
\boldsymbol{\tau}=\mu\left(\nabla \mathbf{u}+\nabla \mathbf{u}^{T}\right)
$$


Under the previously cited assumptions the linear momentum equation, eq. (2-10), reduces to:

$$
\rho \mathbf{g}-\nabla p+\mu \nabla^{2} \mathbf{u}=\rho\left[\frac{\partial \mathbf{u}}{\partial t}+\mathbf{u} \nabla \cdot \mathbf{u}\right]
$$

The eq. (2-12) is known as the Navier-Stokes equation [31] and particular solutions of interest will be displayed on the following sections.

\subsection{2}

\section{Stokes flow}

Stokes flow [25], also known as creeping flow, is characterized by a very viscous liquid or flow under small velocity. This phenomena makes the viscous term $\mu \nabla^{2} \mathbf{u}$ orders of magnitude bigger than the inertia term $\rho \mathbf{u} \nabla \cdot \mathbf{u}$, leading the eq. (2-12) to the following simplification:

$$
\rho \mathbf{g}+\mu \nabla^{2} \mathbf{u}=\nabla p
$$

\subsection{3}

\section{Darcy's Law}

Darcy's law was experimentally derived by Darcy [29] to describe the flow of water through packed sands. Nevertheless Darcy's law also can be derived by averaging the incompressible Navier-Stokes equations for Newtonian fluid flow of nearly constant density [19]. In a more straight-forward and simplistic approach, starting from the eq. (2-13), Darcy's law can be derived assuming that the viscous term of Stokes flow equation is proportional to the velocity, or $\mu \nabla^{2} \mathbf{u}=\mu \mathbf{k}^{-\mathbf{1}} \mathbf{u}$, resulting in:

$$
\mu \mathbf{k}^{-\mathbf{1}} \mathbf{u}+\rho \mathbf{g}-\nabla p=0
$$

For a flow perpendicular to the gravity direction and an isotropic porous media, the permeability tensor reduces to a diagonal tensor with all components equal to $k$ and the eq. (2-14) simplifies to:

$$
u=-\frac{k}{\mu} \nabla p
$$

\subsection{4}

\section{Brinkman's Equation}

A more formal and complete derivation using volume average techniques of Darcy's equation can be found on [32]. When using this approach one term often called Brinkman's correction [33] naturally appears. The StokesBrinkman equation represents the fluid flow in a medium composed by a freeflow and porous media region defined by: 


$$
\nabla p=-\mu \mathbf{k}^{-1} \mathbf{u}+\mu^{*} \nabla^{2} \mathbf{u}
$$

Equation (2-16) holds the Darcy flow equation [29] added the Brinkman's viscous term, $\mu^{*} \nabla^{2} \mathbf{u}$. The $\mu^{*}$ is called Brinkman's viscosity or effective viscosity and has the same unity as the viscosity. With the appropriate choice of parameters it is possible to reduce Brinkman's equation to its end-members, for $k \rightarrow \infty$, the viscous forces are significant and Stokes equation is obtained, whereas for $\mu^{*} \rightarrow 0$, the viscous forces are negligible and Darcy's equation is obtained. 


\section{Numerical Formulation}

\section{1}

\section{The Finite Element Method}

The Finite Element Method (FEM) is a numerical method to solve a Partial Differential Equation (PDE) using a approximate solutions. The FEM requires the PDE to be reformulate in terms of its equivalent variational form. Once the problem is stated like this, an approximate solution can be found for a discrete domain represented by a set of finite elements that is subjected to certain boundary conditions. The approximate solution is the projection of the exact solution in a given function subspace [34].

The ability to handle complex domains and the flexibility to model complex physical phenomena using a common framework makes it appropriate for flow simulations in realistic porous media, such as carbonate rock samples.

\section{2}

\section{The FEniCS Project and DOLFIN Library}

The FEniCS Project is a free ${ }^{1}$ collaborative project for the development of innovative concepts and tools for automated scientific computing, with a particular focus on automated solution of differential equations by finite element methods [35]. Figure 3.1 outlines the FEniCS Project structure highlighting the layout of the different components and how they interact with each other.

DOLFIN is a $\mathrm{C}++/$ Python library that acts as the main user interface of FEniCS [36]. A large part of the functionality of FEniCS is implemented as part of DOLFIN. It provides a problem solving environment for models based on partial differential equations and implements core parts of the functionality of FEniCS, including data structures and algorithms for computational meshes and finite element assembly. To provide a simple and consistent user interface, DOLFIN wraps the functionality of other FEniCS components and external software, and handles the communication between these components.

\footnotetext{
${ }^{1}$ Using the GNU LGPL license published by the Free Software Foundation
} 
Initially the problem is stated in terms of its variational form. Then it is implemented using the Unified Form Language (UFL) which does the finite element method declaration [37]. Next the code is compiled using the FEniCS Form Compiler (FFC) where a low-level code is automatically created [38]. This code will be under the Unified Form-assembly Code (UFC) standards and can be easily accessed using DOLFIN classes [39]. FFC relies on several different back-ends, including FIAT, Instant and FErari. FIAT is a back-end for the evaluation of basis functions [40], Instant is a just-in-time compiler, and FErari is an optimizing back-end. FErari is optional and not needed to use DOLFIN, while the former two are essential parts of the tool-chain. The Viper module is a stand alone plotting utility that is imported alongside DOLFIN. It allows plotting of DOLFIN functions, meshes, finite elements and others based on VTK format [41].

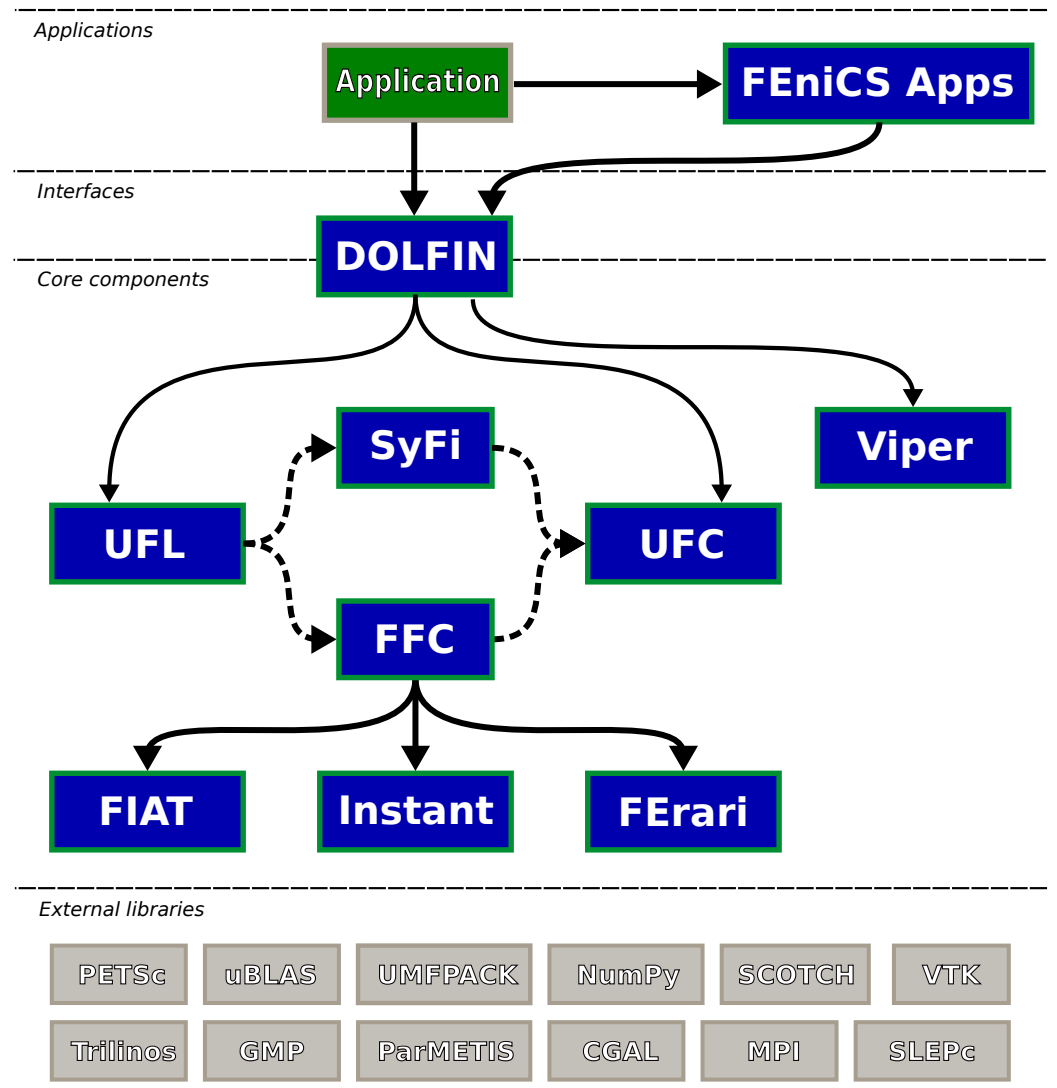

Figure 3.1: Overview structure of the FEniCS Project, from [35].

The next section will introduce the formulation for the Poisson equation in order to give a simple and short introduction on FEniCS syntax and functionality. It will briefly identify the PDE and its boundary conditions, the variational problem reformulated from the PDE problem and the Python routine used to code the variational problem and obtain its solution. The Python program will make use of FEniCS 1.2.0 version and is present at The 
FEniCS Project website [42].

\section{3}

\section{Poisson's Equation}

The Poisson equation for a domain $\Omega \subset \mathbb{R}^{2}$ with boundary $\Gamma \subset \mathbb{R}^{1}$ reads as:

$$
-\nabla^{2} u=f \quad \text { in } \Omega .
$$

where $u=u(x, y), f$ is a known function and the boundary conditions are defined as:

$$
\begin{aligned}
\nabla u \cdot n & =g \quad \text { on } \Gamma_{N}, \\
u & =u_{0} \quad \text { on } \Gamma_{D} .
\end{aligned}
$$

where $g$ and $u_{0}$ are known variables and $n$ denotes the outward directed boundary normal. A variational form of Poisson equation reads as find $u \in V$ such that:

$$
a(u, v)=L(v), \quad \forall v \in V
$$

where $V$ is a suitable function space with $a(u, v)$ and $L(v)$ defined as:

$$
\begin{aligned}
a(u, v) & =\int_{\Omega} \nabla u \cdot \nabla v \mathrm{dx}, \\
L(v) & =\int_{\Omega} f v \mathrm{dx}+\int_{\Gamma_{\mathrm{N}}} \mathrm{g} \mathrm{vds} .
\end{aligned}
$$

The expressions $a(u, v)$ and $L(v)$ are respectively the bilinear and the linear variational forms. It is assumed that all functions in $V$ satisfy the Dirichlet boundary conditions $\left(u=u_{0}\right.$ on $\left.\Gamma_{D}\right)$.

In the present description, the following definitions of the input functions, the domain and the boundaries are considered:

$-\Omega=[0,1] \times[0,1]$

$-\Gamma_{D}=\{(0, y) \cup(1, y) \subset \Gamma\}$;

$-\Gamma_{N}=\{(x, 0) \cup(x, 1) \subset \Gamma\}$;

$-u=u_{0}$

$-g=\sin (5 x)$

$-f=10^{-50 *(x-1 / 2)^{2}+(y-1 / 2)^{2}}$.

The step-by-step description of the assembled routines and solver for the above Poisson equation are described below, the complete code can be found 
in appendix A.1. All code definitions and commands are formatted with the True Type Standard font.

First, the dolfin module is imported:

from dolfin import *

This module is responsible for containing all functions and definitions used herein. The full Python script contains a description header that is not shown here and can be found at appendix A.1. The fact that this listing starts at line 37 is explained by description header length.

The implementation starts with a discrete domain represented by a mesh and a finite element function space $V$ relative to this mesh. The unit square is a standard domain and so the mesh is provided by the class UnitSquareMesh. The mesh consists of $32 \times 32$ squares with each square divided into two triangles:

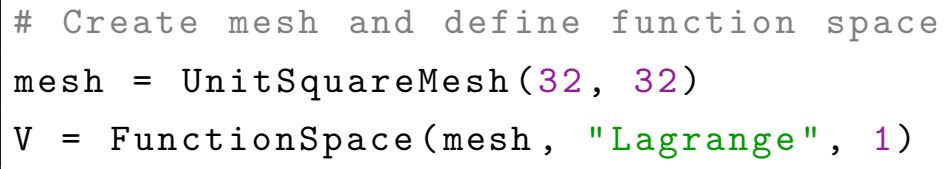

The second argument to FunctionSpace is the finite element family, while the third argument specifies the polynomial degree. Thus, in this case, the space $\mathrm{V}$ consists of first-order, continuous Lagrange finite element functions, or in order words, continuous piecewise linear polynomials.

Next, Dirichlet boundary conditions are considered. A simple Python function returning a boolean, can be used to define the sub-domain for the Dirichlet boundary condition, $\Gamma_{D}$. The function should return True for those points inside the sub-domain and False for the points outside. For this particular case, the points $(x, y)$ such that $x=0$ or $x=1$ are on $\Gamma_{D}$. Note that to avoid rounding-off errors, it is often wise to instead specify $x<\epsilon$ or $x>1-\epsilon$, where $\epsilon$ is a small number, such as machine precision.

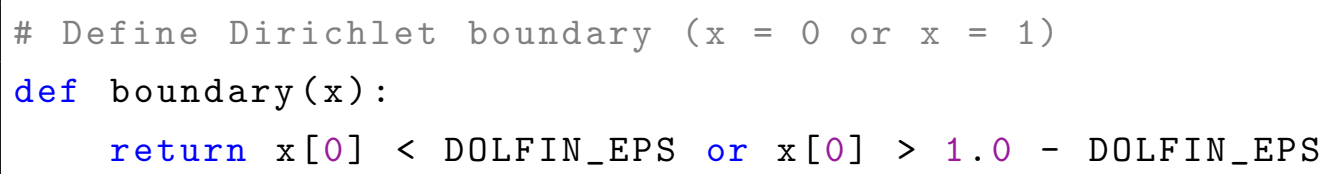

The Dirichlet boundary condition is created using the class DirichletBC and it takes three arguments: the function space where the boundary condition applies to, the value of the boundary condition, and the region of the boundary on which the condition is applied. In this particular example, the function 
space is $\mathrm{V}$, the value of the boundary condition can be represented using a Constant and the Dirichlet boundary is defined immediately. The definition of the Dirichlet boundary condition looks as follows:

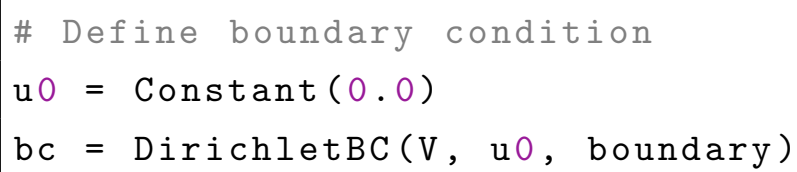

Next, the variational problem is expressed. First, the trial function $u$ and the test function $v$ are specified, both living in the function space $V$. This is done by defining a TrialFunction and a TestFunction on the previously defined FunctionSpace, v.

Further, the term $f$ and the boundary normal derivative $g$ are involved in the variational forms, and hence these are specified. Both $f$ and $g$ are given by simple mathematical formulas, and can be easily declared using the Expression class. Note that the strings defining $f$ and $g$ use $\mathrm{C}++$ syntax since, for efficiency, DOLFIN will generate and compile $\mathrm{C}++$ code for these expressions at run-time.

The bilinear form $a(u, v)$ and the linear form $L(u)$ are written using UFL operators. In summary, this reads:

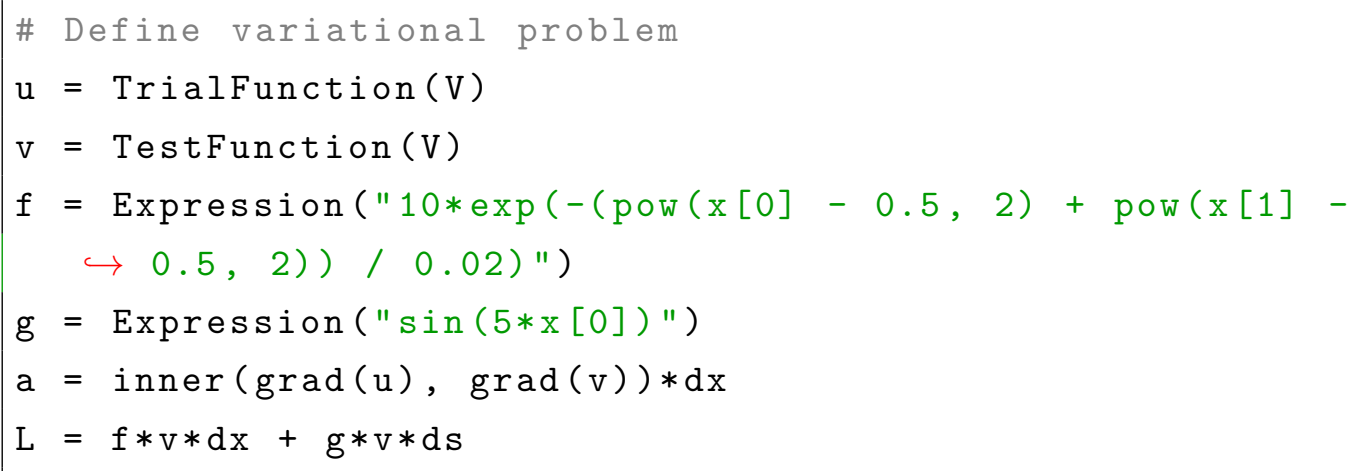

Now, the solution of the variational problem is considered. First, the function $u$ is defined to represent the solution. Upon initialization, it is simply set to the zero function. A class Function represents a function living in a finite element function space. Next, the solve function is called with the arguments $\mathrm{a}==\mathrm{L}, \mathrm{u}$ and $\mathrm{bc}$ as follows:

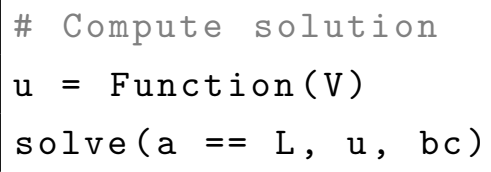


The function $\mathrm{u}$ will be modified during the call to solve. The default settings for solving a variational problem have been used. However, if desired the solution process can be controlled in much more detail.

A Function can be manipulated in various ways, in particular, it can be plotted and saved to file. Here the solution is outputted to a VTK file [41], using the suffix .pvd, for later visualization and also is plotted using the plot command:

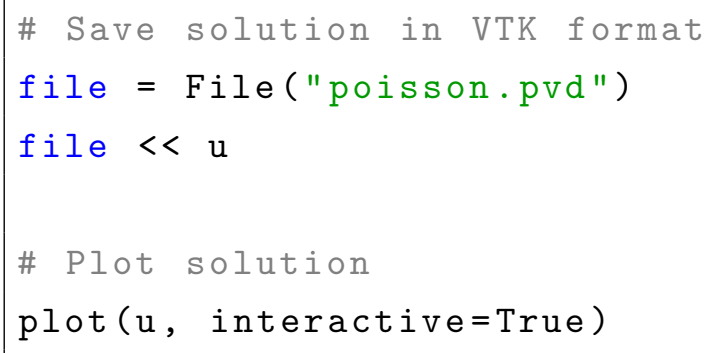

The solution for $u$ can be seen in fig. 3.2. This figure was created using the saved file poisson.pvd and the software distribution ParaView [43].
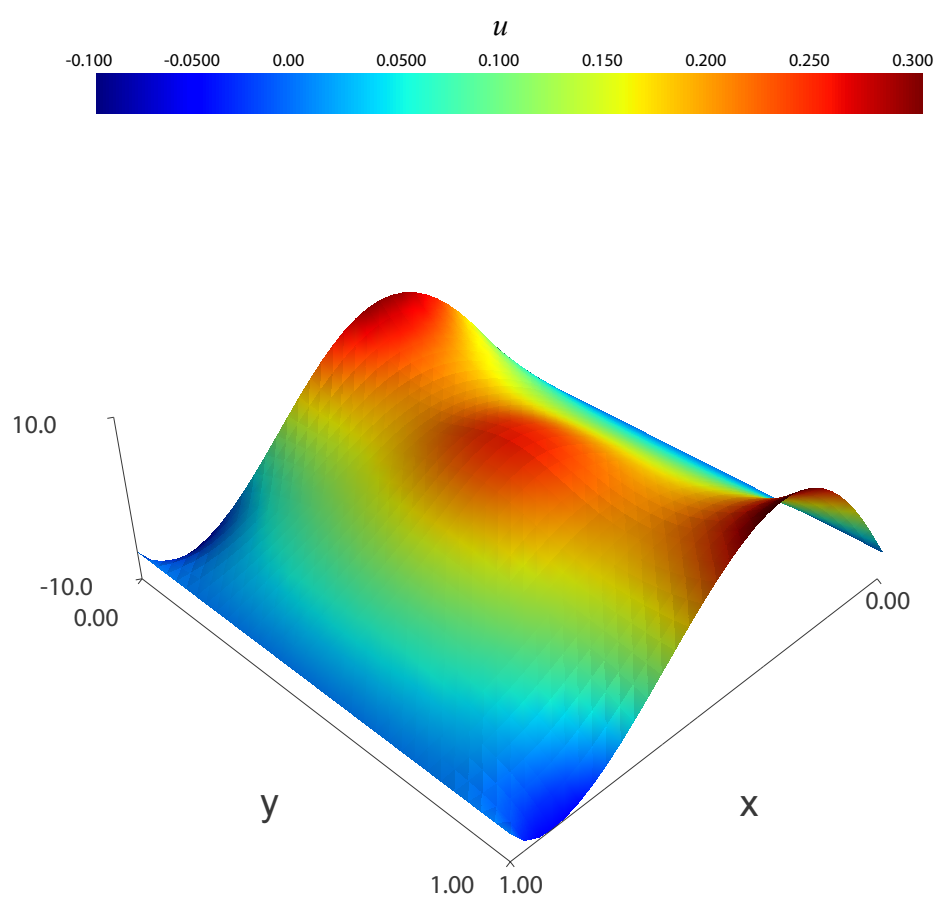

Figure 3.2: Solution for Poisson equation described at eq. (A-1) and generated using the FEniCS package [35]. 


\section{4}

\section{Darcy's Flow Equation}

\subsection{1}

\section{Darcy's Variational Formulation}

Consider Darcy's flow eq. (2-15), described in section 2.3.3 defined for a homogeneous and isotropic medium of permeability $k$ and fluid viscosity $\mu$ :

$$
\begin{aligned}
\mathbf{u}=-\frac{k}{\mu} \nabla p & \text { in } \Omega, \\
\nabla \cdot \mathbf{u}=-f & \text { in } \Omega,
\end{aligned}
$$

with boundary conditions defined as

$$
\begin{aligned}
\mathbf{u} \cdot n & =u_{0} \quad \text { on } \Gamma_{D}, \\
p & =p_{\text {in }} \text { on } \Gamma_{p_{\text {in }}}, \\
p & =p_{\text {out }} \text { on } \Gamma_{p_{\text {out }}} .
\end{aligned}
$$

where $\Gamma_{p_{\text {in }}}$ and $\Gamma_{p_{\text {out }}} \in \Gamma$ are the boundaries where $p_{\text {in }}$ is the specified entry pressure and $p_{\text {out }}$ the is specified out pressure and $u_{0}$ the velocity pointing out of the Dirichlet boundary $\Gamma_{D}$.

A variational form of the eq. (A-5) reads as find $(\mathbf{u}, p) \in \mathbf{V} \times Q$ such that:

$$
\begin{array}{rlrl}
a_{D}(\mathbf{u}, \mathbf{v})+b(\mathbf{v}, p) & =\int_{\Omega} \mathbf{f} \cdot \mathbf{v} d x & & \forall \mathbf{v} \in \mathbf{V}, \\
b(\mathbf{u}, q) & =-\int_{\partial \Omega} g \mathbf{v} \cdot \mathbf{n} d s & \forall q \in Q
\end{array}
$$

where the bilinear variational forms $a_{D}$ and $b$ are defined as

$$
\begin{aligned}
a_{D}(\mathbf{u}, \mathbf{v}) & =\int_{\Omega} \frac{\mu}{k} \mathbf{u} \cdot \mathbf{v} d x \\
b(\mathbf{v}, p) & =-\int_{\Omega} \nabla \cdot \mathbf{v} p d x
\end{aligned}
$$

\subsection{2}

\section{Darcy's Finite Element Implementation}

The computational implementation of Darcy's flow equation eq. (A-5) doesn't differ in many aspects from the implementation described at section 3.3 
for Poisson equation. Therefore only particular listings are going to be detailed herein. The full implementation is present at appendix A.2.

Different from what was chosen in section 3.3, a new type of finite element basis is going to be employed, named Brezzi-Douglas-Marini (BDM) elements [44]. A stable choice of function spaces for Darcy's flow problem is the combination of order $k \mathrm{BDM}$ elements with an order $k-1$ discontinuous Galerkin elements (DG) [45]. These function spaces is created combining two FunctionSpace:

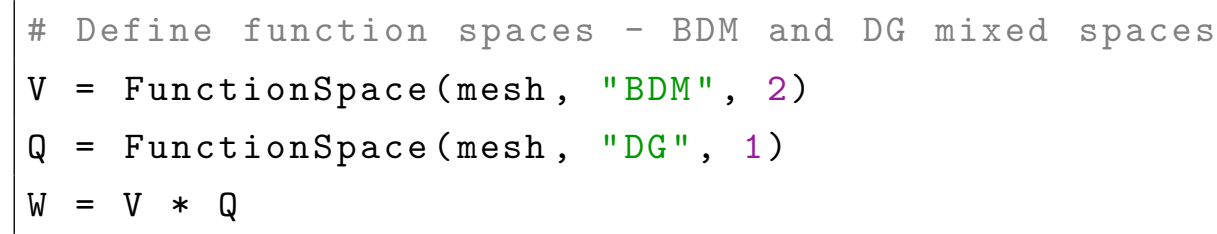

The first argument of the Functionspace class is the mesh, the second a string specifying the element family and the third specifies the polynomial degree of the element. The string 'BDM' stands for Brezzi-Douglas-Marini and the 'DG' for discontinuous Galerkin elements. The UFL user manual contains a list of all available finite element families and more details. The $*$ operator creates a mixed (product) space $\mathrm{W}$ from the two previously defined spaces $\mathrm{V}$ and $Q$ spaces.

Before continuing the input parameters used along with the variational formulation are created:

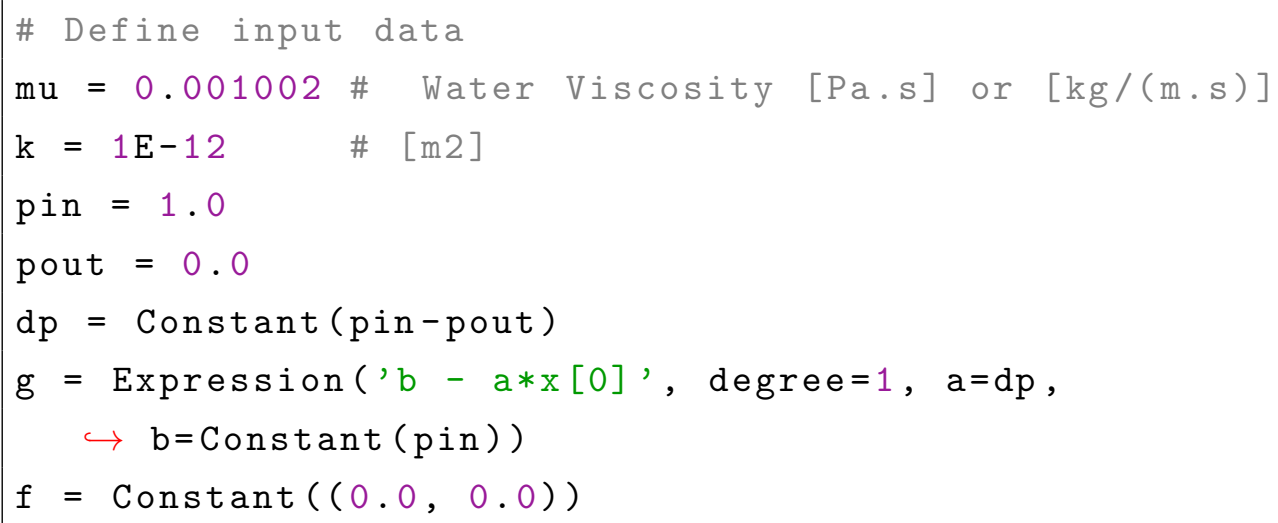

The mu and $\mathrm{k}$ stands respectively for the fluid's viscosity $\mu$ and the porous medium permeability $k$. The variables pin, pout and dp are respectively the entry pressure, out pressure and pressure difference. These variables are constants defined using the Constant function for efficiency. The last two variables, $g$ and $f$ are created to specify the surface and body forces acting 
on the domain $\Omega$ as an Expression and a Constant function.

Darcy's flow equation described at eq. (A-8) can now be defined directly from it's variational forms a and L to be solved:

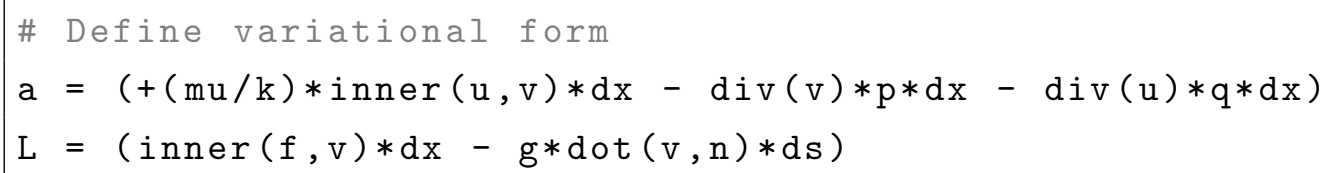

Appendix A.2 contains the full implementation and can be used to solve the pressure driven flow in a porous medium that was described in this section.

\subsection{3}

\section{Darcy's Example}

Using the finite element implementation of Darcy's equation presented in section 3.4.2 it is possible to solve pressure and velocity distribution for particular cases. Figure 3.3(a) shows a porous media domain of size $l=1 \mathrm{~m}$ that is divided in two sub-domains each with $l_{1 / 2}=l / 2=1 / 2 \mathrm{~m}$. The subdomain $\Omega_{D 1}$ has permeability $k=10 \mathrm{mD}$ and $\Omega_{D 2}$ permeability $k=100 \mathrm{mD}$. The fluid viscosity is set to $\mu=0.001002 \mathrm{~Pa} \cdot \mathrm{s}$. The domain is subjected to a pressure gradient with the left face, $x=0 \mathrm{~m}$, set to $p_{i n}=1 \mathrm{~Pa}$ and the right face, $x=1 \mathrm{~m}$, set to $p_{\text {out }}=0 \mathrm{~Pa}$. The other faces are set to symmetry condition. The domain is modeled as $\left[n_{i} \cdot n_{j}\right]=[10 \cdot 10]$ squares with crossed triangles and fig. 3.3(b) shows this numerical mesh.

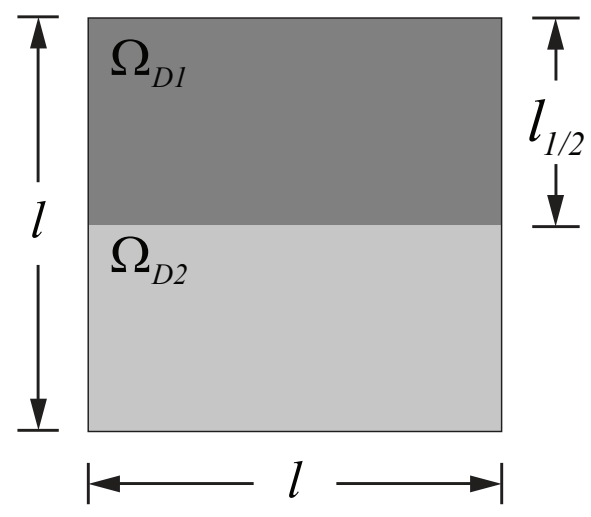

3.3(a): Porous domain.

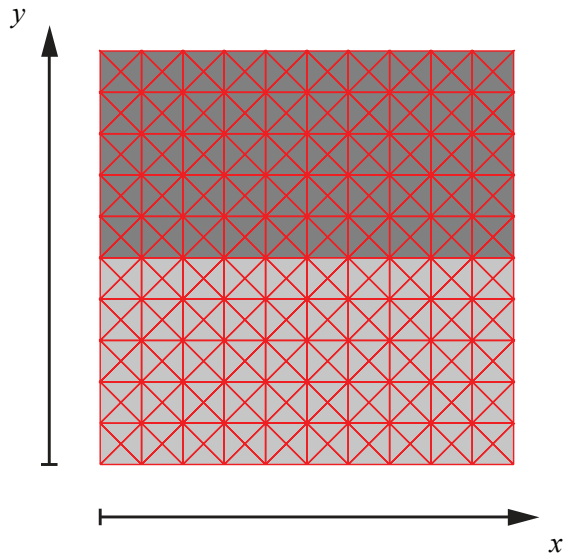

3.3(b): Triangular mesh.

Figure 3.3: Porous medium with different permeability. The domain size is $l$ and is subdivided in two domains each with height $l_{1 / 2}=l / 2$. The sub-domain $\Omega_{D 1}$ has permeability $k=10 \mathrm{mD}$ and $\Omega_{D 2}$ permeability $k=100 \mathrm{mD}$. 


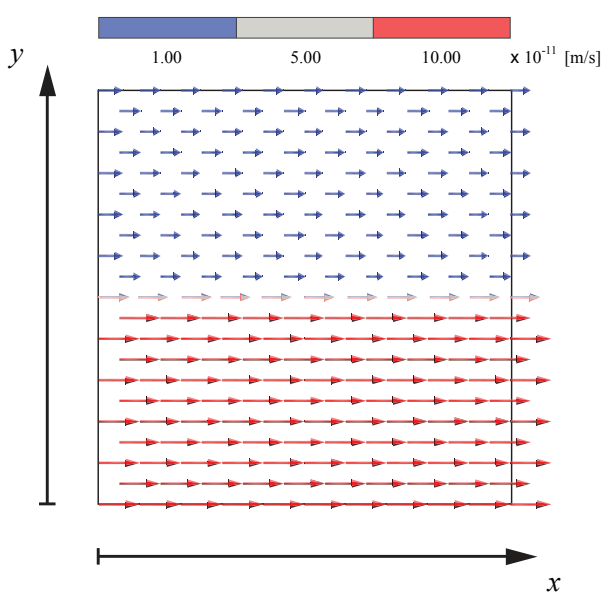

3.4(a): Velocity.

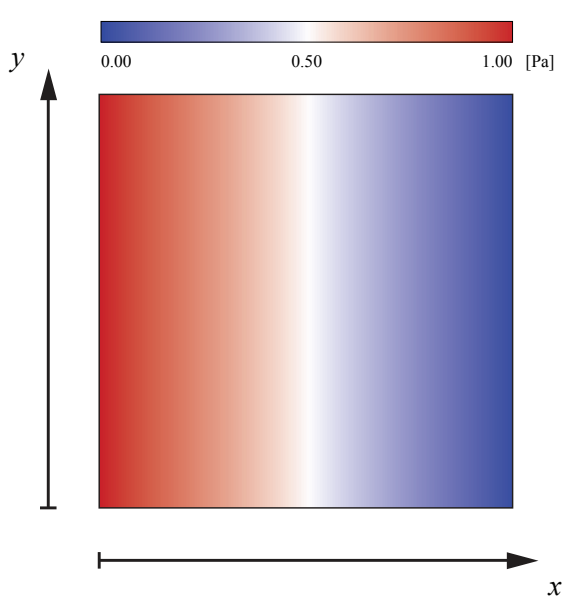

3.4(b): Pressure.

Figure 3.4: Darcy's flow equation solved for a porous medium of permeability $k$ and fluid viscosity $\mu$.

Figures 3.4(a) and 3.4(b) shows respectively the velocity vectors and the pressure distribution. Figure 3.4(a) shows how each sub-domain permeability controls the velocity. The average velocity at sub-domain $\Omega_{D 1}$ is $\bar{u}_{D 1}=$ $1.00 \times 10^{-11} \mathrm{~m} / \mathrm{s}$, the average velocity near the interface between both subdomains is $\bar{u}_{\text {inter }}=5.00 \times 10^{-11} \mathrm{~m} / \mathrm{s}$ and the average velocity at sub-domain $\Omega_{D 2}$ is $\bar{u}_{D 2}=10.00 \times 10^{-11} \mathrm{~m} / \mathrm{s}$. Those results are numerically consistent to what could be derived from eq. (2-15).

\section{5}

\section{Stokes' Flow Equation}

\subsection{1}

\section{Stokes' Variational Formulation}

Consider Stokes's flow equation eq. (2-13) described in section 2.3.2 for a fluid with viscosity $\mu$ and under steady laminar flow:

$$
\begin{aligned}
\rho \mathbf{g}+\mu \nabla^{2} \mathbf{u}=\nabla p & \text { in } \Omega, \\
\nabla \cdot \mathbf{u}=-f & \text { in } \Omega,
\end{aligned}
$$

with boundary conditions defined as

$$
\begin{aligned}
\mathbf{u} \cdot n & =u_{0} \quad \text { on } \Gamma_{D}, \\
p & =p_{\text {in }} \quad \text { on } \Gamma_{p_{\text {in }}}, \\
p & =p_{\text {out }} \text { on } \Gamma_{p_{\text {out }}} .
\end{aligned}
$$


where $\Gamma_{p_{\text {in }}}$ and $\Gamma_{p_{\text {out }}} \in \Gamma$ are the boundaries where $p_{\text {in }}$ the entry pressure and $p_{\text {out }}$ the out pressure are specified and $u_{0}$ the velocity pointing out of the Dirichlet boundary $\Gamma_{D}$.

A variational form of the $(\mathrm{A}-9)$ reads as find $(\mathbf{u}, p) \in \mathbf{V} \times Q$ such that:

$$
\begin{array}{rlrl}
a_{S}(\mathbf{u}, \mathbf{v})+b(\mathbf{v}, p) & =\int_{\Omega} \mathbf{f} \cdot \mathbf{v} d x & & \forall \mathbf{v} \in \mathbf{V}, \\
b(\mathbf{u}, q) & =-\int_{\partial \Omega} g \mathbf{v} \cdot \mathbf{n} d s & \forall q \in Q
\end{array}
$$

where the bilinear variational forms $a_{S}$ and $b$ are defined as

$$
\begin{aligned}
a_{S}(\mathbf{u}, \mathbf{v}) & =\int_{\Omega} \nabla \mathbf{u}: \nabla \mathbf{v} d x \\
b(\mathbf{v}, p) & =-\int_{\Omega} \nabla \cdot \mathbf{v} p d x
\end{aligned}
$$

\subsection{2}

\section{Stokes' Finite Element Implementation}

As for the Darcy's flow equation described in section 3.4.2, the computational implementation of Stokes's flow equation eq. (A-9) is similar to those already presented, therefore only particular listings are going to be detailed herein. The full implementation is present at appendix A.3.

Different from what was chosen in section 3.4, a new type of finite element basis is going to be employed but with the same input parameters where common. The Taylor-Hood elements [46] consists of an order $k$ element for the velocity space and an order $l$, with $l<k$, element for the pressure space. The Taylor-Hood element is created combining a VectorFunctionSpace with a FunctionSpace:

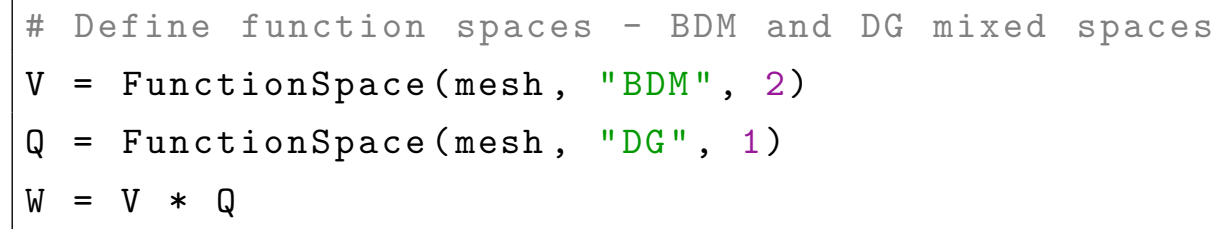

Both VectorFunctionSpace and FunctionSpace classes are defined in a similar manner. The first argument is the mesh, the second a string specifying the element family and the third specifies the polynomial degree of the element. The string 'CG' stands for Continuous Galerkin, implying the standard 
Lagrange family of elements. Instead of ' $\mathrm{CG}$ ', the family of elements could be set using the string 'Lagrange'. With degree 1, the linear Lagrange element is selected.

Stokes's flow equation described at eq. (A-12) can now be defined directly from it's variational forms a and L to be solved:

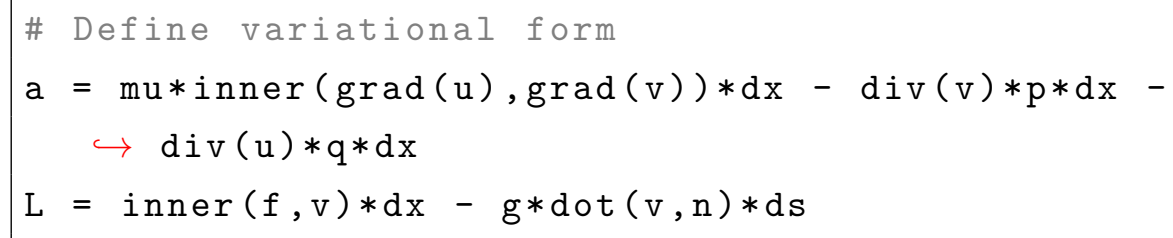

Appendix A.3 contains the full implementation and can be used to solve the pressure driven flow in a porous medium that was described in this section.

\subsection{3}

\section{Stokes' Example}

Using the finite element implementation of Stokes' equation present at section 3.5.2 it is possible to solve pressure and velocity distribution for particular cases. Figure 3.5(a) shows the flow between parallel plates of size $l=1 \mathrm{~m}$ bounded by two walls, one on the bottom, $y=0 \mathrm{~m}$, and the other on the top, $y=1 \mathrm{~m}$, of the domain. The fluid viscosity is set to $\mu=0.001002$ $\mathrm{Pa} \cdot \mathrm{s}$. The domain is subjected to a pressure gradient with the left face, $x=0$ $\mathrm{m}$, set to $p_{\text {in }}=1 \mathrm{~Pa}$ and the right face, $x=1 \mathrm{~m}$, set to $p_{\text {out }}=0 \mathrm{~Pa}$. The domain is modeled as $\left[n_{i} \cdot n_{j}\right]=[10 \cdot 10]$ squares with crossed triangles and fig. 3.5(b) shows this numerical mesh.

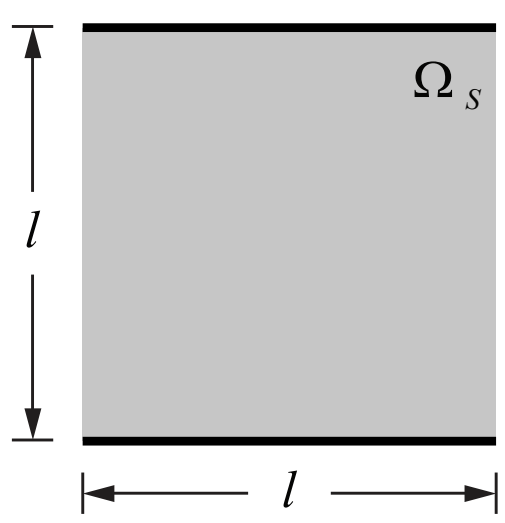

3.5(a): Parallel plates.

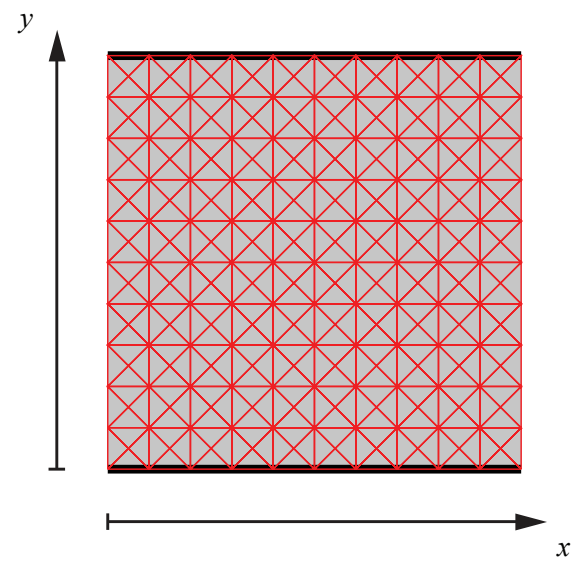

3.5(b): Triangular mesh.

Figure 3.5: Parallel plates domain. The domain size is $l$ and is bounded by two walls, one on top and the other on the bottom of the domain. 
Figures 3.6(a) and 3.6(b) shows respectively the velocity vectors and the pressure distribution. Figure 3.4(a) shows the expected parabolic velocity profile this flow. The maximum velocity at the domain $\Omega_{S}$ is $u_{\max }=125.00$ $\mathrm{m} / \mathrm{s}$. This value is numerically consistent to what could be derived solving eq. (2-13) with the same boundary conditions.

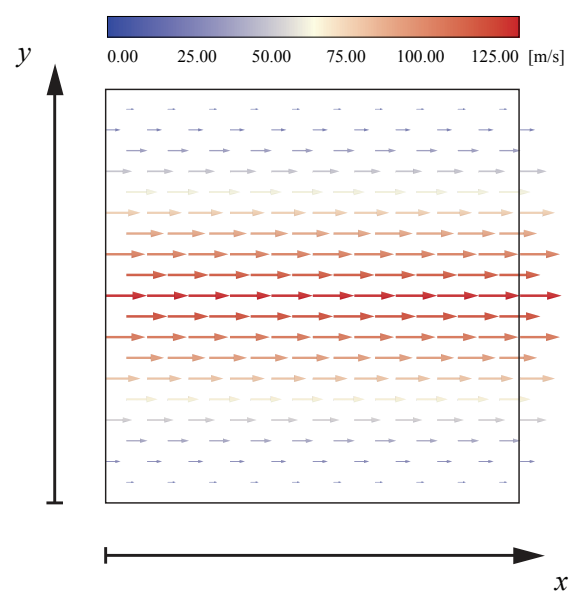

3.6(a): Velocity.

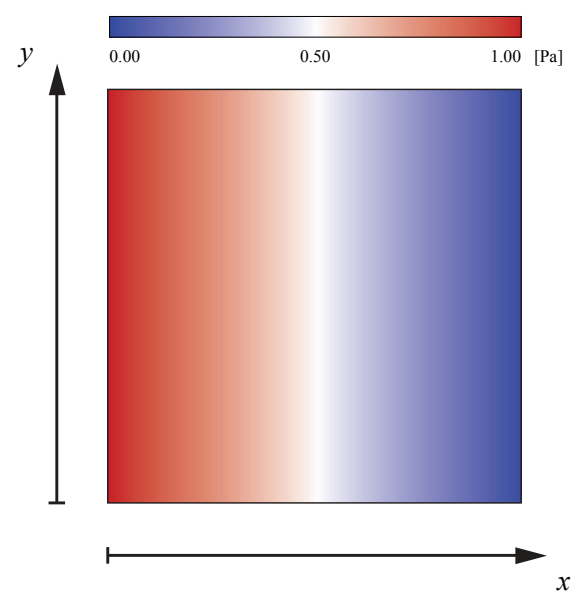

3.6(b): Pressure.

Figure 3.6: Stokes' flow equation solved for parallel plates and fluid viscosity $\mu$.

\section{6}

\section{Brinkman's Flow Equation}

\subsection{1}

\section{Brinkman's Variational Formulation}

Consider Brinkman's flow equation eq. (2-16) described in section 2.3.4, for a homogeneous and isotropic medium of permeability $k$ and fluid viscosity $\mu$ :

$$
\begin{aligned}
-\mu^{*} \nabla^{2} \mathbf{u}+\nabla p+\mu \mathbf{k}^{-1} \mathbf{u}=\mathbf{f} & \text { in } \Omega \\
\nabla \cdot \mathbf{u}=g & \text { in } \Omega
\end{aligned}
$$

with boundary conditions defined as

$$
\begin{aligned}
& \mathbf{u} \cdot n=u_{0} \quad \text { on } \Gamma_{D}, \\
& p=p_{\text {in }} \text { on } \Gamma_{p_{\text {in }}}, \\
& p=p_{\text {out }} \text { on } \Gamma_{p_{\text {out }}} \text {. }
\end{aligned}
$$


where $\Gamma_{p_{\text {in }}}$ and $\Gamma_{p_{\text {out }}} \in \Gamma$ are the boundaries where $p_{\text {in }}$ the entry pressure and $p_{\text {out }}$ the out pressure are specified and $u_{0}$ the velocity pointing out of the Dirichlet boundary $\Gamma_{D}$.

A variational form of the $(\mathrm{A}-9)$ reads as find $(\mathbf{u}, p) \in \mathbf{V} \times Q$ such that:

$$
\begin{array}{rlrl}
a_{B}(\mathbf{u}, \mathbf{v})+b(\mathbf{v}, p) & =\int_{\Omega} \mathbf{f} \cdot \mathbf{v} d x & & \forall \mathbf{v} \in \mathbf{V}, \\
b(\mathbf{u}, q) & =-\int_{\partial \Omega} g \mathbf{v} \cdot \mathbf{n} d s & \forall q \in Q
\end{array}
$$

where the bilinear forms $a_{B}$ and $b$ are defined as

$$
\begin{aligned}
a_{B}(\mathbf{u}, \mathbf{v}) & =\int_{\Omega} \mu \nabla \mathbf{u}: \nabla \mathbf{v} d x+\int_{\Omega} \frac{\mu}{k} \mathbf{u} \cdot \mathbf{v} d x \\
b(\mathbf{v}, q) & =\int_{\Omega} \nabla \cdot \mathbf{v} q d x
\end{aligned}
$$

As described at section 2.3.4, the Brinkman's flow equation, eq. (A13), delineates two different flows: the Stokes, or free-fluid domain, and the Darcy, or porous medium domain. For Stokes domain, $k \rightarrow \infty$ and $a_{B} \rightarrow a_{S}=\int_{\Omega} \mu \nabla \mathbf{u} \cdot \nabla \mathbf{v} d x$, whereas for Darcy domain, $\mu^{*} \rightarrow 0$ and $a_{B} \rightarrow a_{D}=\int_{\Omega} \frac{\mu}{k} \mathbf{u} \cdot \mathbf{v} d x$.

\subsection{2}

\section{Brinkman's Finite Element Implementation}

As for the Darcy's and Stokes' flow equations described in sections 3.4.2 and 3.5.2, the computational implementation of Brinkman's flow eq. (A-13) is similar to the previous cases. As previously, only particular listings are going to be detailed herein. The full implementation is presented at appendix A.4.

The main difference arises from the solution of Brinkman's equation that needs to be split accordingly to the domain that will be solved. This can be done either on a pre-processing step, marking specific regions of the domain that will correspond to either a Stokes or Darcy domain or marking the domain directly on the code implementation. If done on a pre-processing step, the marked domain will be imported using the function MeshFunction. If marked along with the code implementation, a new class has to be created: 
When evaluated, this class will return True or False if inside, or outside a specified radius of 0.25 units.

As in section 3.5, the Taylor-Hood elements [46] are used along with the same input parameters where common. Taylor-Hood elements were successfully employed for the Stokes-Brinkman problem [47], stabilized equal order methods were also used with equal or better results but are not going to be detailed here [48].

DOLFIN predefines the measures $\mathrm{dx}$, ds and $\mathrm{dS}$ representing integration over cells, exterior facets and interior facets, respectively. New metrics have to be created to be consistent with the new marked domains. This is obtained using the function Measure:

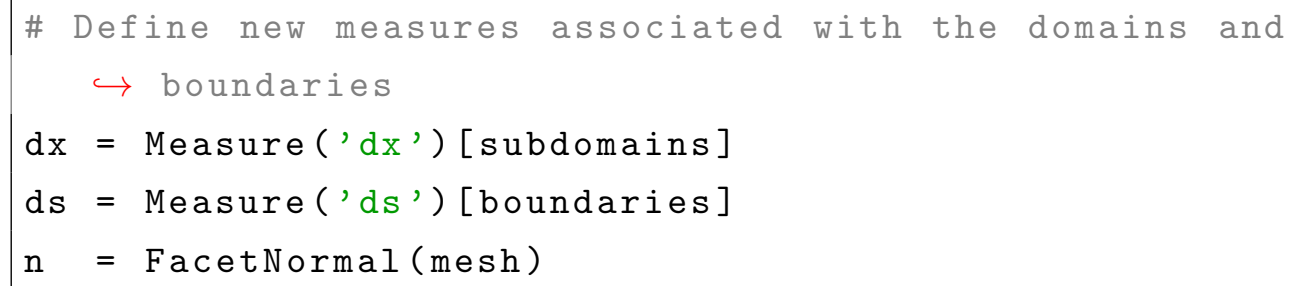

Brinkman's flow equation described in eq. (A-16) can now be defined directly from it's variational forms a and $\mathrm{L}$ to be solved:

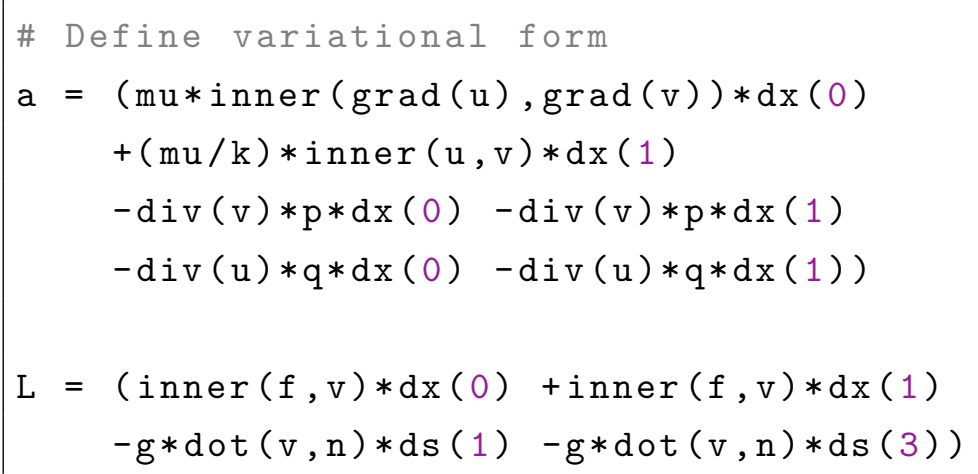

Appendix A.4 contains the full implementation and can be used to solve the pressure and velocity fields in a porous medium with a circular free-fluid region.

\subsection{3}

\section{Brinkman's Example}

Using the finite element implementation of Brinkman's equation present at section 3.6.2 it is possible to solve pressure and velocity distribution for particular cases. Figure 3.7(a) shows a square porous media domain of size 
$l=1 \mathrm{~m}$ that is divided in two sub-domains. The sub-domain $\Omega_{D}$ is porous domain and has permeability of $k=100 \mathrm{mD}$ and $\Omega_{S}$ is a free-fluid domain. The fluid viscosity is set to $\mu=0.001002 \mathrm{~Pa} \cdot \mathrm{s}$. The domain is subjected to a pressure gradient with the left face, $x=0 \mathrm{~m}$, set to $p_{i n}=1 \mathrm{~Pa}$ and the right face, $x=1 \mathrm{~m}$, set to $p_{\text {out }}=0 \mathrm{~Pa}$. The other faces are set to symmetry condition. The domain is modeled as $\left[n_{i} \cdot n_{j}\right]=[10 \cdot 10]$ squares with crossed triangles and fig. 3.7(b) shows this numerical mesh.

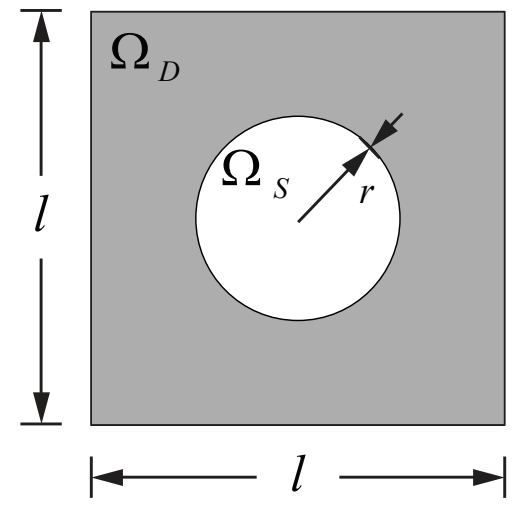

3.7(a): Porous domain.

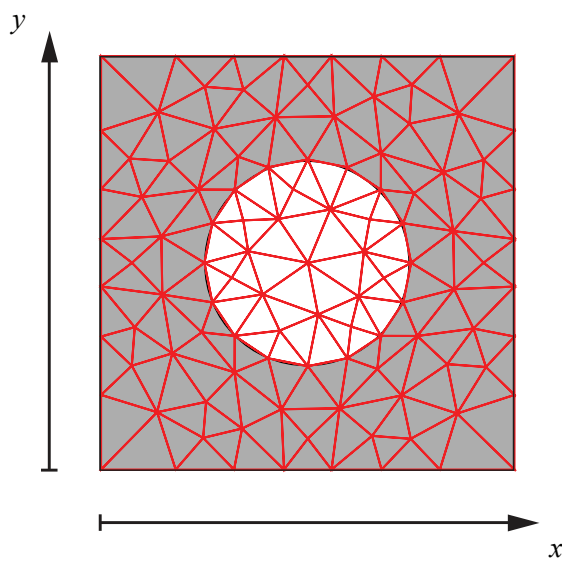

3.7(b): Triangular mesh.

Figure 3.7: Porous medium with circular inclusion. The domain size is $l$ and is subdivided in two domains. The sub-domain $\Omega_{D}$ is porous domain and has permeability of $k=100 \mathrm{mD}$ and $\Omega_{S}$ is a free-fluid domain.

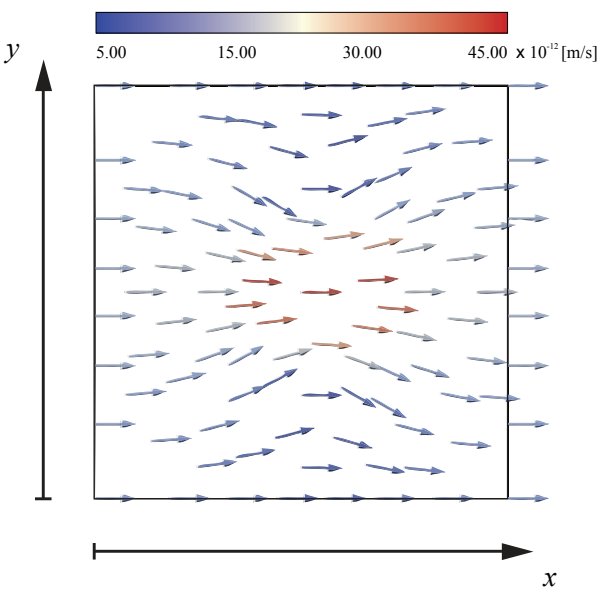

3.8(a): Velocity.

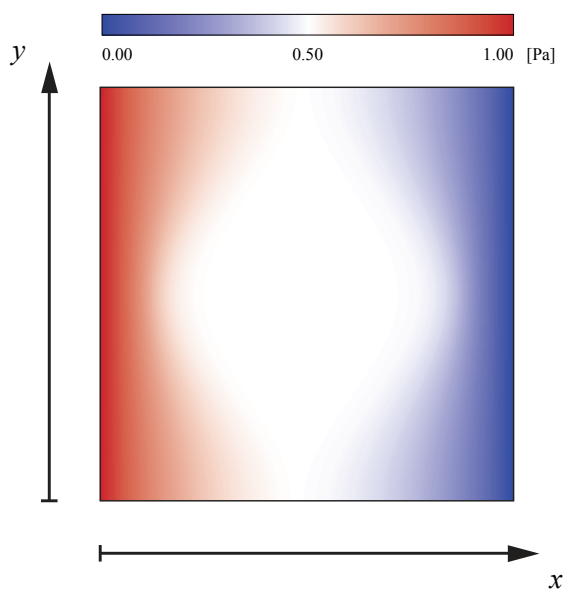

3.8(b): Pressure.

Figure 3.8: Brinkman's flow equation solved for an isotropic medium of permeability $k$ and fluid viscosity $\mu$ with the presence of a circular free-fluid region. 
Figures 3.8(a) and 3.8(b) shows respectively the velocity vectors and the pressure distribution. The fig. 3.8(a) shows the velocity diverging from the porous media to the free-fluid region. Similar models will be stressed to study the influence of the vug shape, size and distribution in the next chapter, chapter 4 . 


\section{Results}

A periodic cell with an arbitrary vug shape is shown at fig. 4.1. The cell size is defined as $l$ and its given dimensions equal to $[0,1] \times[0,1]$. The cell domain, $\Omega$, is divided in two sub-domains, $\Omega_{D}$ and $\Omega_{S}$ with $\Omega=\Omega_{D} \cup \Omega_{S}$. These sub-domains represents respectively the Darcy and Stokes medium or, in other words, the porous and free-flow medium, the last corresponding to the vug region. The cell boundary, $\Gamma$, is divided in 4 segments, $\Gamma_{1}, \Gamma_{2}, \Gamma_{3}$ and $\Gamma_{4}$, respectively corresponding to the boundaries at left, top, right and bottom of the domain. The vug's sub-domain $\Omega_{S}$ is modeled as a free-flow medium by assuming that in eq. (2-16) the effective viscosity is $\mu^{*}=\mu$ and that the permeability $k \rightarrow \infty$.

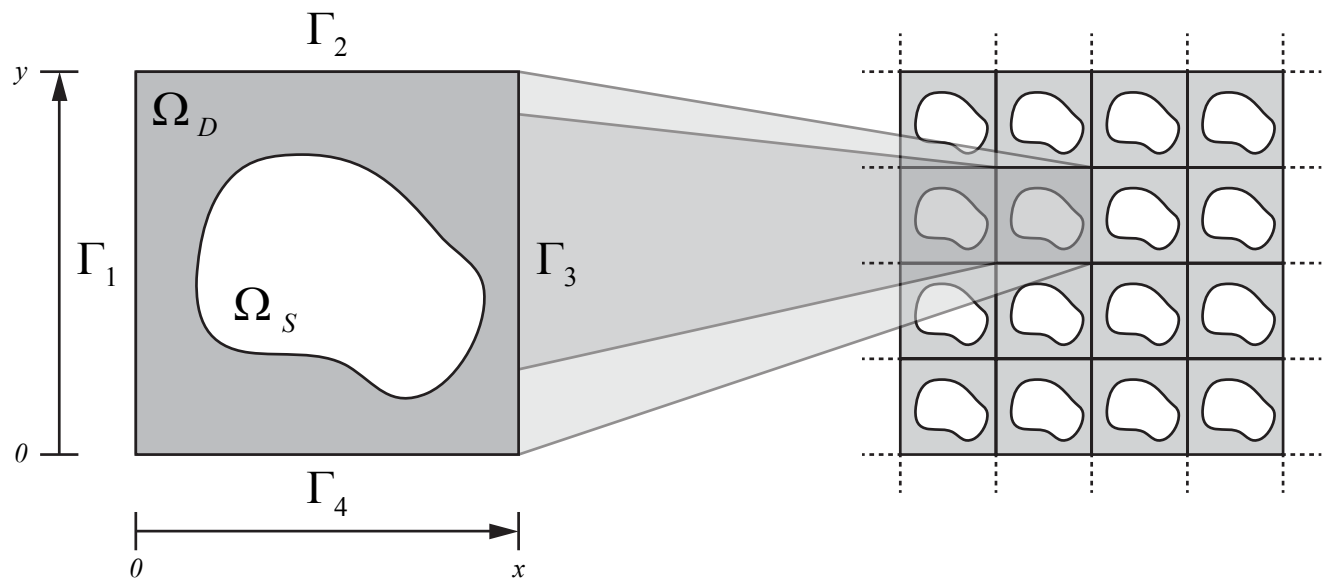

Figure 4.1: Periodic cell showing a vug of arbitrary shape. The domain, $\Omega$, is composed of two sub-domains, $\Omega_{D}$, with the subscript $D$ corresponding to the Darcy sub-domain, and $\Omega_{S}$, with the subscript $S$ corresponding to the Stokes sub-domain, the last corresponding to the vug region. The boundaries, $\Gamma$, is split into $\Gamma_{1}, \Gamma_{2}, \Gamma_{3}$ and $\Gamma_{4}$, respectively corresponding to the left, top, right and bottom of the domain

The periodic cell shown in fig. 4.1 shares the same geometric description as all other periodic cells discussed on the following sections, with the main difference lying in the vug shape or size. 


\section{1}

\section{A Layered Medium}

The layered medium corresponds to a porous matrix containing a conduit that communicates opposite faces of the given periodic cell, this conduit can be interpreted as a fracture. Figure 4.2 shows the periodic cell for a layered medium with size $l \times l$ and fracture aperture $a$. As previously described, $\Omega_{D}$ and $\Omega_{S}$ respectively corresponds to the Darcy and Stokes sub-domains. As can be seen on fig. 4.2(b), the discrete mesh elements are refined towards the interface between both sub-domains and finer at $\Omega_{S}$ than $\Omega_{D}$.

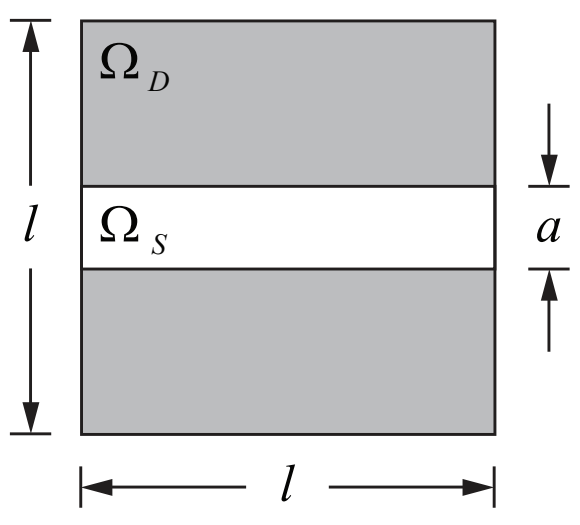

4.2(a): Layered domain.

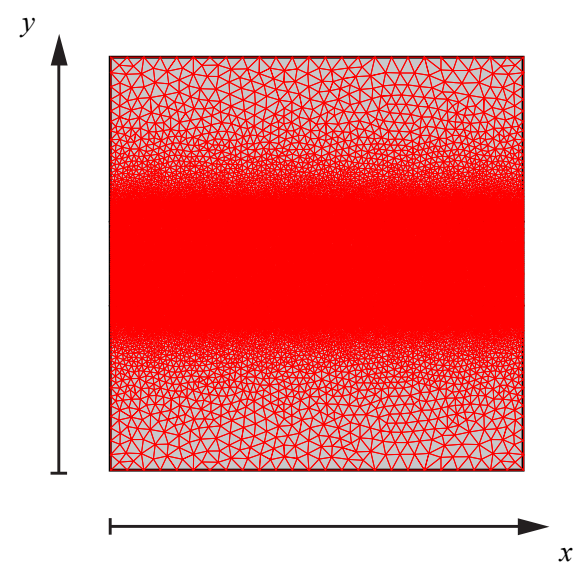

4.2(b): Layered mesh.

Figure 4.2: A periodic cell for the layered medium of size $l \times l$ and conduit aperture $a$. The $\Omega_{D}$ and $\Omega_{S}$ respectively stands for Darcy and Stokes subdomains. The discrete mesh is refined towards the sub-domains interface and finer inside $\Omega_{S}$ sub-domain.

\subsection{1}

\section{The Effect of the Fracture Aperture}

Using the layered medium previously described and shown in fig. 4.2(a), it is possible to study to what extent the fracture aperture $a$ and porous matrix permeability $k_{\text {matrix }}$ impacts on the periodic cell's permeability. The first comparison arrives from the derived fracture permeability. When combining Stokes equation eq. (2-13) with Darcy's equation eq. (2-14), one can estimate the average permeability of a fracture in a given domain using the following relationship:

$$
k=\frac{a^{2}}{12}
$$

This relationship expresses the permeability of a fracture as a function of the fracture aperture $a$ and it is derived making the flow calculated from 
Darcy's equation equal to the laminar flow between stationary parallel plates. It is also known as the cubic law permeability and can be found in different formats [49].

Table 4.1 contains the estimated values of permeability for both the fracture permeability calculated using eq. (4-1) and the estimated permeability scaled-up from the layered periodic cell shown at fig. 4.2(a) using matrix permeability $k_{\text {matrix }}=10 \mathrm{mD}$. Figure 4.3 illustrate these results.

Table 4.1: Effect of the varying aperture $a / l$ for the estimated permeability of the periodic layered domain shown at fig. 4.2(a) and the fracture permeability defined by eq. (4-1).

\begin{tabular}{cccc}
\hline $\boldsymbol{a} / \boldsymbol{l}$ & $\boldsymbol{u}_{\boldsymbol{a v \boldsymbol { g }}}[\mathrm{m} / \mathbf{s}]$ & $\boldsymbol{k}_{\boldsymbol{a b s}}[\boldsymbol{m} \boldsymbol{D}]$ & $\boldsymbol{k}_{\text {fracture }}[\boldsymbol{m D}]$ \\
\hline 0.05 & $2.12 \mathrm{E}-01$ & $1.06 \mathrm{E}+10$ & $2.08 \mathrm{E}+11$ \\
0.10 & $1.65 \mathrm{E}+00$ & $8.25 \mathrm{E}+10$ & $8.33 \mathrm{E}+11$ \\
0.20 & $1.33 \mathrm{E}+01$ & $6.65 \mathrm{E}+11$ & $3.33 \mathrm{E}+12$ \\
0.30 & $4.49 \mathrm{E}+01$ & $2.25 \mathrm{E}+12$ & $7.50 \mathrm{E}+12$ \\
0.40 & $1.06 \mathrm{E}+02$ & $5.33 \mathrm{E}+12$ & $1.33 \mathrm{E}+13$ \\
0.50 & $2.08 \mathrm{E}+02$ & $1.04 \mathrm{E}+13$ & $2.08 \mathrm{E}+13$ \\
0.60 & $3.59 \mathrm{E}+02$ & $1.80 \mathrm{E}+13$ & $3.00 \mathrm{E}+13$ \\
0.70 & $5.70 \mathrm{E}+02$ & $2.86 \mathrm{E}+13$ & $4.08 \mathrm{E}+13$ \\
0.80 & $8.51 \mathrm{E}+02$ & $4.27 \mathrm{E}+13$ & $5.33 \mathrm{E}+13$ \\
0.90 & $1.21 \mathrm{E}+03$ & $6.07 \mathrm{E}+13$ & $6.75 \mathrm{E}+13$ \\
0.95 & $1.43 \mathrm{E}+03$ & $7.15 \mathrm{E}+13$ & $7.52 \mathrm{E}+13$ \\
\hline
\end{tabular}

In general the fracture permeability $k_{\text {fracture }}$ defined by eq. $(4-1)$ is somewhat bigger than the estimated permeability from the layered medium. For small fracture apertures the estimated permeability is almost one order of magnitude smaller than the fracture permeability, however this difference becomes smaller as the fracutre aperture increases.

\subsection{2}

\section{The Effect of the Matrix Permeability}

Next it is shown the impact of the matrix permeability over the scaledup estimated permeability for the layered periodic cell. A brief comparison is made with a parallel plate flow [23], with velocity profile defined by:

$$
u=\frac{1}{2 \mu} \frac{d p}{d x} y(d-y)
$$

The geometry of the periodic cell is the same previously described on fig. 4.2 (a) but with aperture fixed at $a / l=0.20$. Table 4.2 contains the scaledup estimated permeability values when varying the matrix permeability. It also contains the average velocity for the layered domain $u_{\text {avg }}$ and the average velocity for a parallel plates flow $u_{\text {plates }}$ using eq. (4-2). The scaled-up estimated 


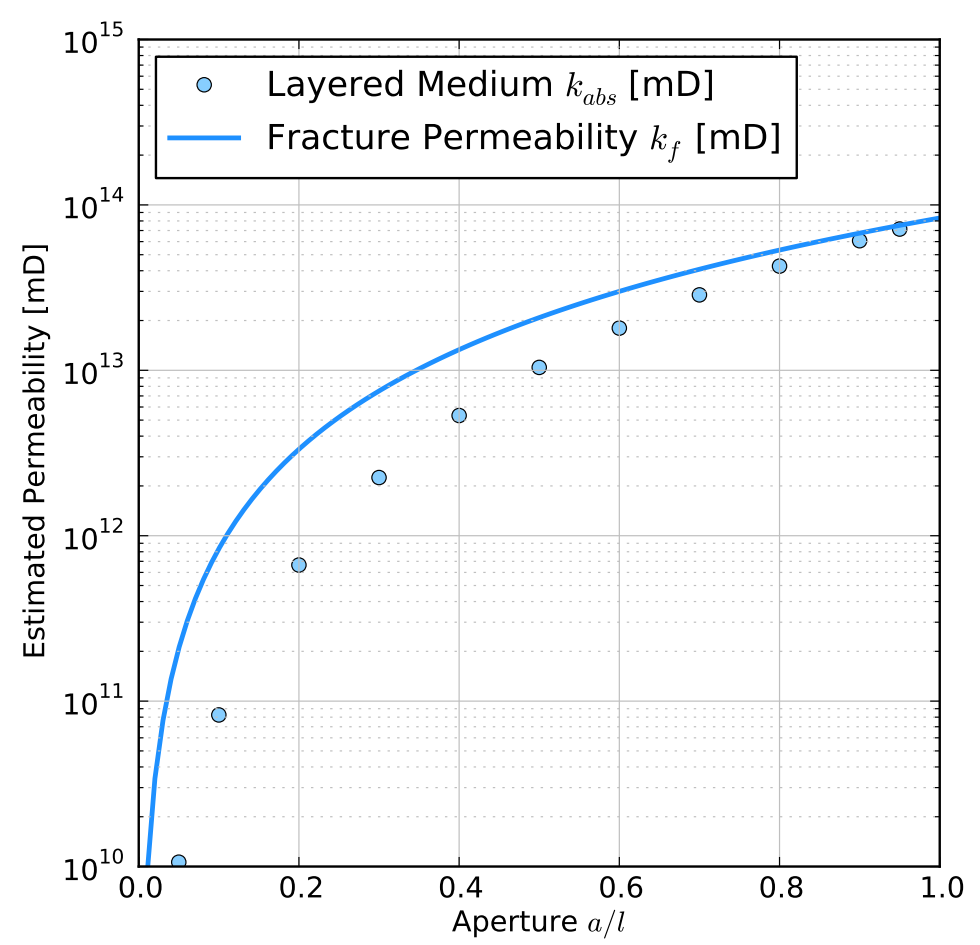

Figure 4.3: Comparison between the estimated permeability for the periodic layered cell, the blue points, and the fracture permeability, the continuous blue line. The $x$-axis shows the fracture aperture $a / l$ and the $y$-axis shows the estimated absolute permeability.

permeability is not significantly influenced by the matrix permeability, a change of five orders of magnitude didn't influence the estimated permeability.

Table 4.2: Effect of the varying matrix permeability $k_{\text {matrix }}$ over $\Omega_{D}$ sub-domain for the periodic layered domain shown at fig. 4.2(a). The table contains the average velocity for the layered domain $u_{\text {avg }}$ and the average velocity for a parallel plates flow $u_{\text {plates }}$ using eq. (4-2).

\begin{tabular}{cccc}
\hline $\boldsymbol{k}_{\boldsymbol{m a t r i x}}[\boldsymbol{m D}]$ & $\boldsymbol{u}_{\boldsymbol{a v g}}[\boldsymbol{m} / \boldsymbol{s}]$ & $\boldsymbol{u}_{\text {plates }}[\boldsymbol{m} / \boldsymbol{s}]$ & $\boldsymbol{k}_{\boldsymbol{a b s}}[\boldsymbol{m D}]$ \\
\hline $1.00 \mathrm{E}-02$ & 13.2734 & 13.3067 & $6.65 \mathrm{E}+11$ \\
$1.00 \mathrm{E}-01$ & 13.2734 & 13.3067 & $6.65 \mathrm{E}+11$ \\
$1.00 \mathrm{E}+00$ & 13.2734 & 13.3067 & $6.65 \mathrm{E}+11$ \\
$1.00 \mathrm{E}+01$ & 13.2734 & 13.3067 & $6.65 \mathrm{E}+11$ \\
$1.00 \mathrm{E}+02$ & 13.2734 & 13.3067 & $6.65 \mathrm{E}+11$ \\
$1.00 \mathrm{E}+03$ & 13.2734 & 13.3067 & $6.65 \mathrm{E}+11$ \\
\hline
\end{tabular}

Figure 4.4 shows the velocity profile for $k_{\text {matrix }}=1 \mathrm{mD}$, fig. $4.4(\mathrm{a})$, and $k_{\text {matrix }}=100 \mathrm{mD}$, fig. $4.4(\mathrm{a})$, and highlights the velocity profile around the interface between the porous and free-fluid medium. The solid black line shows the estimated velocity profile and the blue thick line the profile for 
parallel plates flow. The zoomed-in detail emphasizes that the velocity profile inside the $\Omega_{D}$ sub-domain follows the changes of the matrix permeability with the same order of magnitude. The velocity profile inside the free-flow fracture matches the velocity profile for the parallel plate flow and only distorts the velocity at the vicinities of the porous media sub-domain.
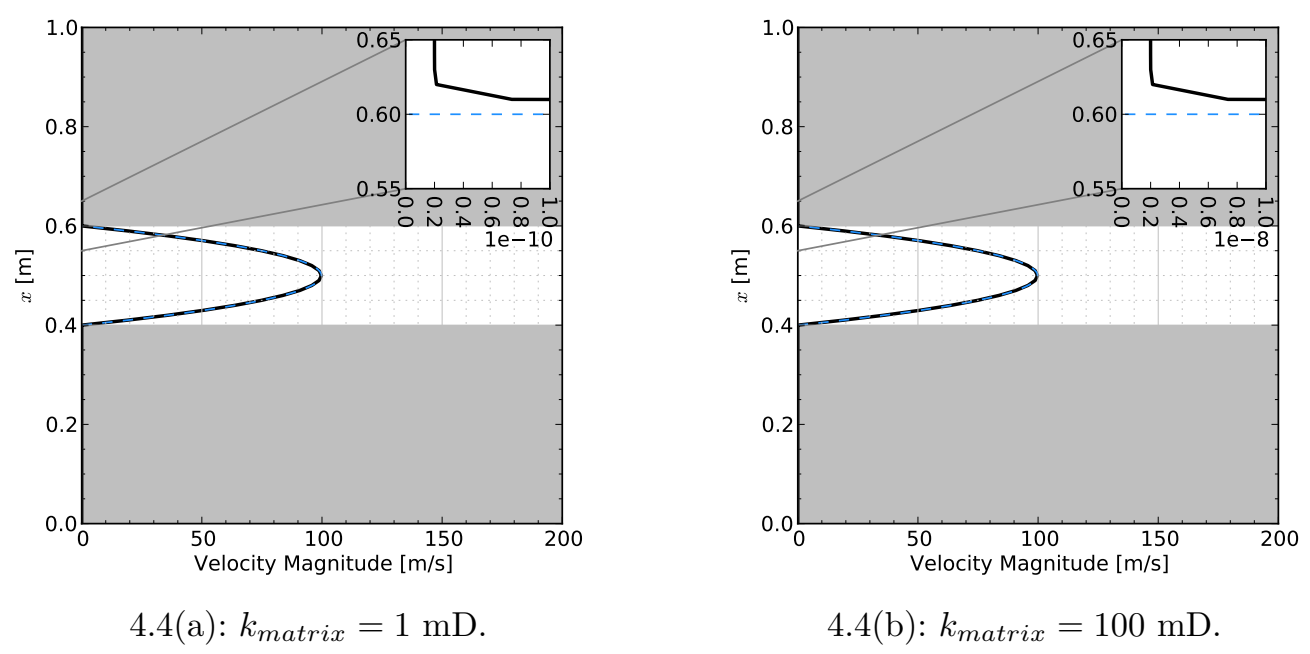

Figure 4.4: Velocity profile for a line crossing $[0.5,0.5] \times[0.0,1.0]$ over the the layered cell $4.2(\mathrm{a})$ with conduit aperture of $a / l=0.20$ highlighting the interface between $\Omega_{D}$ and $\Omega_{S}$. The solid black line shows the estimated velocity profile and the blue tick line the profile for a parallel plates flow.

\section{2}

\section{A Porous Medium with Vugs}

The geometric shape impact is studied using four different periodic cells. The cells share the same geometry described in fig. 4.1 and follows the ratio $\Omega_{D} / \Omega=1 / 4$, despite being out of scale on the same. Figures $4.5(\mathrm{a})$ to $4.5(\mathrm{~d})$ contains all the four studied shapes, circle-, square-, hexagonal- and crossshaped vugs respectively. Although not shown here, the mesh refinement for the periodic cells at fig. 4.1 follows the same behaviour presented in fig. 4.2(b), refined towards the interface between both sub-domains and finer in $\Omega_{S}$ than $\Omega_{D}$.

\subsection{1}

\section{The Effect of the Size of the Vug}

Using the previously described circle-shaped vug, described in fig. 4.5(a), it is possible to study until what extent the vug radius $r$ and porous matrix permeability $k_{\text {matrix }}$ impacts on the periodic cell's scaled-up estimated permeability. Table 4.3 contains the estimated values of the scaled-up 


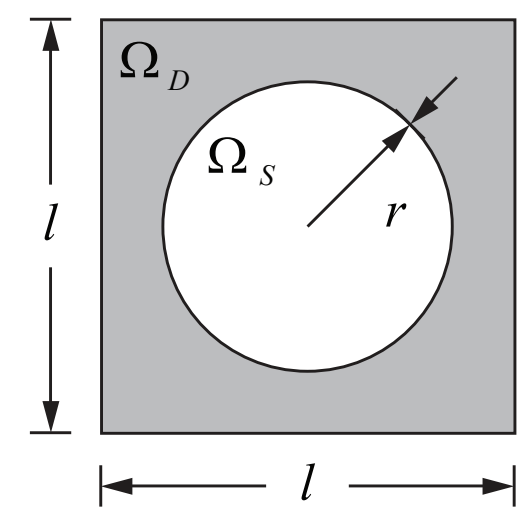

4.5(a): Circular vug.

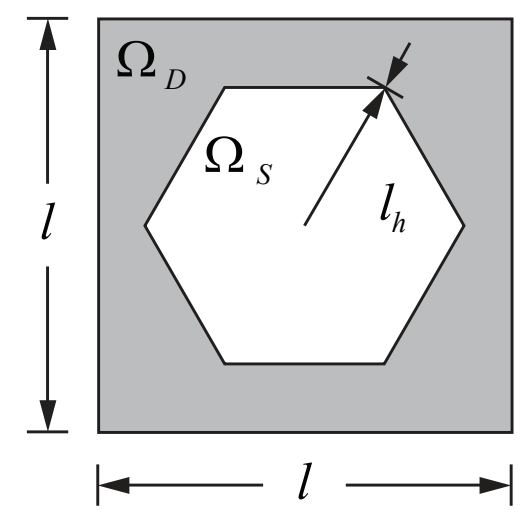

4.5(c): Hexagonal vug.

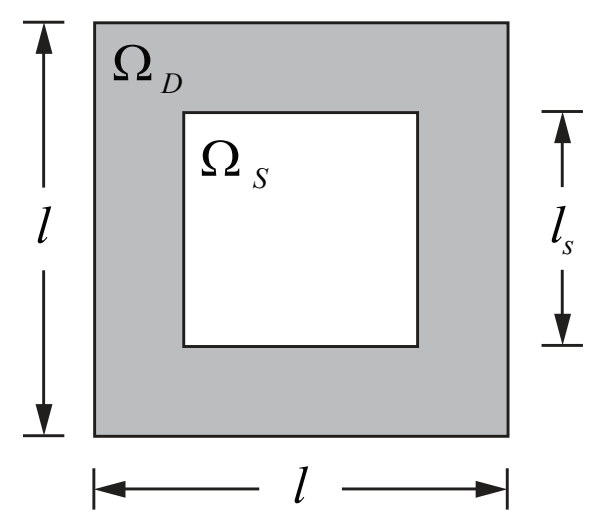

4.5(b): Square vug.

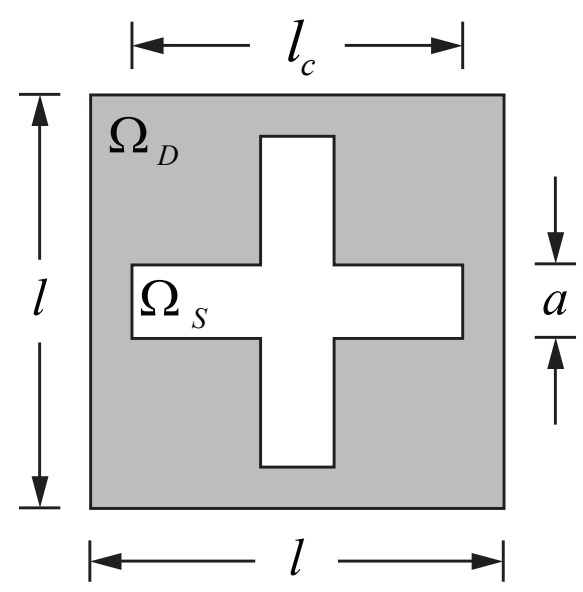

4.5(d): Cross vug.

Figure 4.5: Four different vugs shape with periodic cell size $l$. The circle radius at fig. 4.5(a) is defined as $r$. The square side size at fig. 4.5(b) is defined as $l$. The radius of the circumscribed hexagon at fig. $4.5(\mathrm{c})$ is $l_{h}$. The cross at fig. $4.5(\mathrm{~d})$ extension is $l_{e}$ and the width $a$. All cells respects the ratio $\Omega_{D} / \Omega=1 / 4$. The $\Omega_{D}$ and $\Omega_{S}$ respectively stands for Darcy and Stokes sub-domains. The figures are out of scale and are only used for illustrative matters.

permeability for the vug radius with matrix permeability fixed at $k_{\text {matrix }}=10$ $\mathrm{mD}$.

The estimated scaled-up permeability slowly increases for vug's diameter smaller than half of the periodic cell's size and rapidly increase after that. The estimated permeability significantly increases once the vug size is nearly the same as the periodic cell's size, fig. 4.3 plots these results. 
Table 4.3: Permeability results for different radius of a circular-shaped vug with medium permeability fixed at $k_{\text {matrix }}=10 \mathrm{mD}$

\begin{tabular}{cccc}
\hline $\boldsymbol{r} / \boldsymbol{l}$ & $\boldsymbol{u}_{\boldsymbol{a v \boldsymbol { g }}}[\boldsymbol{m} / \boldsymbol{s}]$ & $\boldsymbol{k}_{\boldsymbol{a b s} \boldsymbol{s}}[\boldsymbol{m} \boldsymbol{D}]$ & $\boldsymbol{k}_{\boldsymbol{a b s}} / \boldsymbol{k}_{\boldsymbol{m a t r i x}}$ \\
\hline 0.050 & $2.03 \mathrm{E}-10$ & 10.16 & 1.02 \\
0.100 & $2.13 \mathrm{E}-10$ & 10.65 & 1.07 \\
0.150 & $2.30 \mathrm{E}-10$ & 11.52 & 1.15 \\
0.200 & $2.57 \mathrm{E}-10$ & 12.87 & 1.29 \\
0.250 & $2.97 \mathrm{E}-10$ & 14.88 & 1.49 \\
0.300 & $3.57 \mathrm{E}-10$ & 17.89 & 1.79 \\
0.350 & $4.52 \mathrm{E}-10$ & 22.62 & 2.26 \\
0.400 & $6.19 \mathrm{E}-10$ & 31.01 & 3.10 \\
0.450 & $1.02 \mathrm{E}-09$ & 51.10 & 5.11 \\
0.495 & $4.03 \mathrm{E}-09$ & 201.99 & 20.20 \\
\hline
\end{tabular}

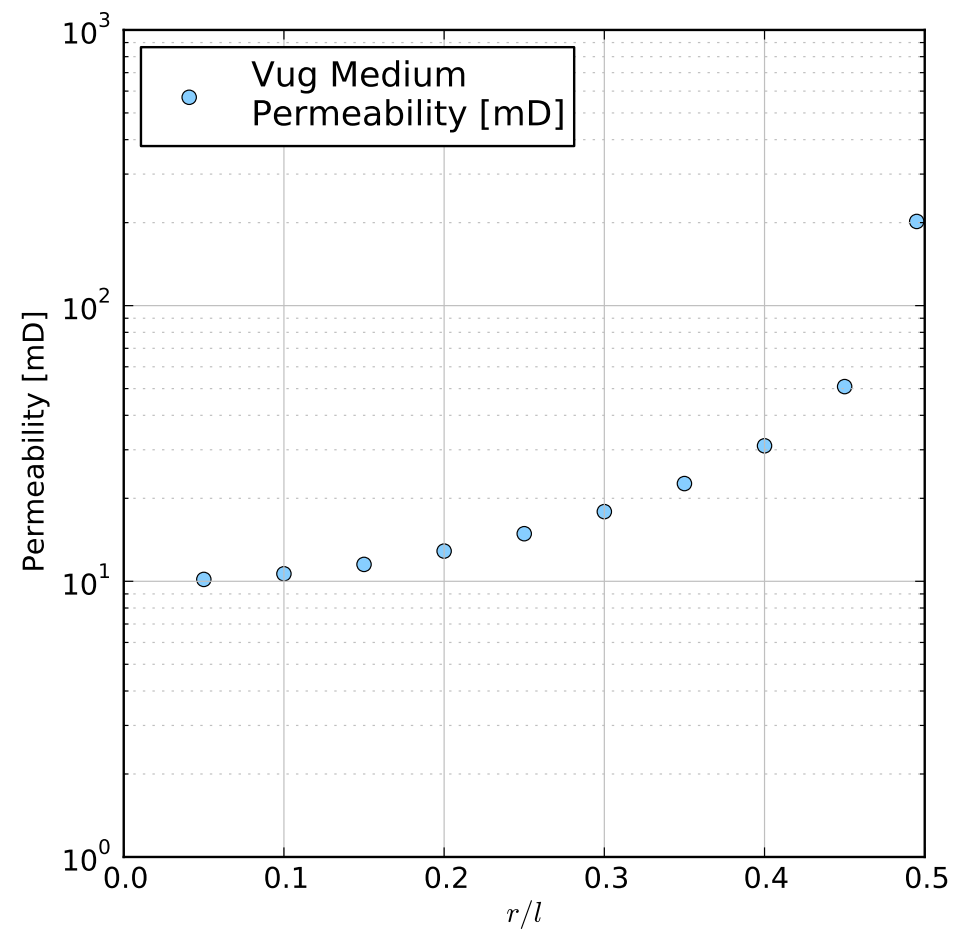

Figure 4.6: Effect of varying radius at a circle-shaped vug. The $x$-axis shows the length $r / l$ and the $y$-axis shows the estimated absolute permeability for the periodic cell.

\subsection{2}

\section{The Effect of the Vug Shape}

Using the periodic cells described in fig. 4.5 it is possible to study the impact of the vug shape in the scaled-up permeability estimation. In order to keep a consistent comparison, the sub-domains area of the periodic cells are equal and set to $A_{\Omega_{D}} / A_{\Omega}=1 / 4$. Table 4.4 shows the scaled-up permeability 
estimative for the studied vug shapes.

Table 4.4: Effect of vug shape for varying matrix permability $k_{\text {matrix }}$.

\begin{tabular}{ccccc}
\hline $\boldsymbol{k}_{\boldsymbol{m a t} \boldsymbol{r} \boldsymbol{x}}$ & $\begin{array}{c}\text { Circle } \\
\boldsymbol{k}_{\boldsymbol{a b s}}\end{array}$ & $\begin{array}{c}\text { Hexagon } \\
\boldsymbol{k}_{\boldsymbol{a b s}}\end{array}$ & $\begin{array}{c}\text { Square } \\
\boldsymbol{k}_{\boldsymbol{a b s}}\end{array}$ & $\begin{array}{c}\text { Cross } \\
\boldsymbol{k}_{\boldsymbol{a b s}}\end{array}$ \\
\hline $1.00 \mathrm{E}-02$ & $1.67 \mathrm{E}-02$ & $1.69 \mathrm{E}-02$ & $1.73 \mathrm{E}-02$ & $5.19 \mathrm{E}-02$ \\
$1.00 \mathrm{E}-01$ & $1.67 \mathrm{E}-01$ & $1.69 \mathrm{E}-01$ & $1.73 \mathrm{E}-01$ & $5.19 \mathrm{E}-01$ \\
$1.00 \mathrm{E}+00$ & $1.67 \mathrm{E}+00$ & $1.69 \mathrm{E}+00$ & $1.73 \mathrm{E}+00$ & $5.19 \mathrm{E}+00$ \\
$1.00 \mathrm{E}+01$ & $1.67 \mathrm{E}+01$ & $1.69 \mathrm{E}+01$ & $1.73 \mathrm{E}+01$ & $5.19 \mathrm{E}+01$ \\
$1.00 \mathrm{E}+02$ & $1.67 \mathrm{E}+02$ & $1.69 \mathrm{E}+02$ & $1.73 \mathrm{E}+02$ & $5.19 \mathrm{E}+02$ \\
$1.00 \mathrm{E}+03$ & $1.67 \mathrm{E}+03$ & $1.69 \mathrm{E}+03$ & $1.73 \mathrm{E}+03$ & $5.19 \mathrm{E}+03$ \\
\hline
\end{tabular}

All studied cases have scaled-up permeability of the same order of magnitude as the matrix permeability. The scaled-up permeability increases as the vug forms a channel connecting opposite faces of the periodic cell. For all simulated cases the presence of the vug increased the estimated permeability with the cross shaped vug being holding the most significant increase. Figure 4.7 summarizes these observations in a chart.

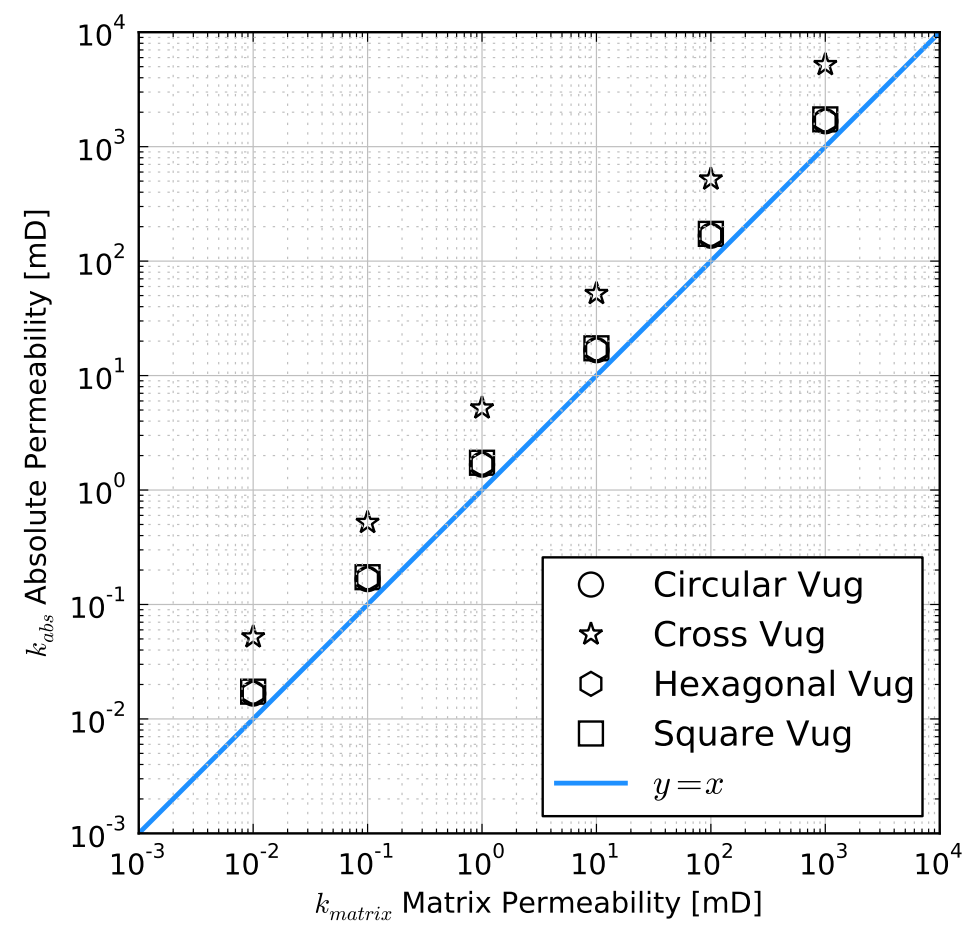

Figure 4.7: Effect of different vug shape. The $x$-axis shows the matrix permeability and the $y$-axis shows the estimated absolute permeability for the periodic cell.

Figure 4.8 exemplifies the pressure and velocity field for the periodic cells present in fig. 4.5. Both the pressure and velocity field are symmetric. 

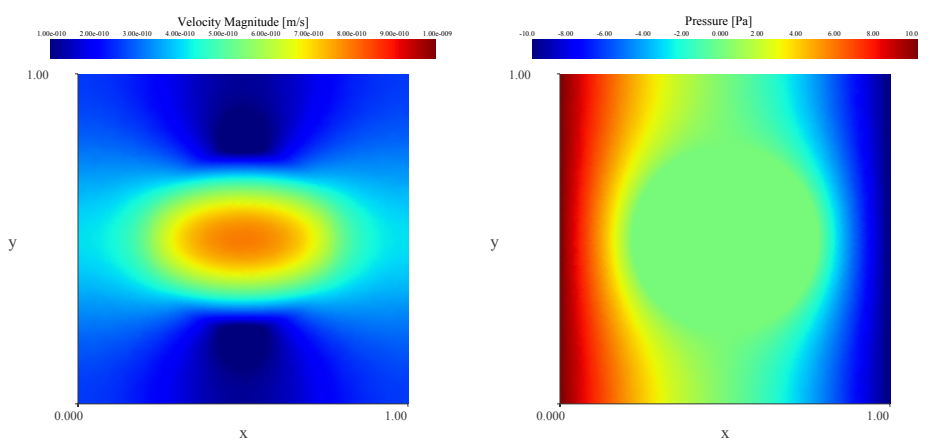

4.8(a): Circular-shaped vug.
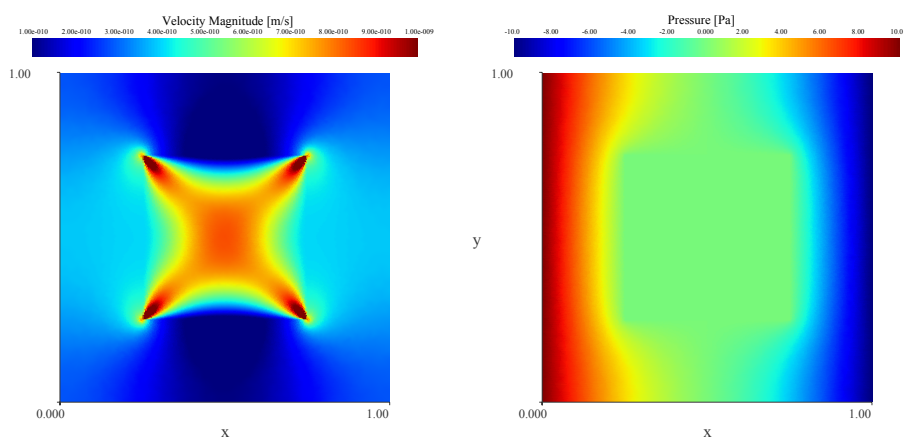

4.8(b): Square-shaped vug.
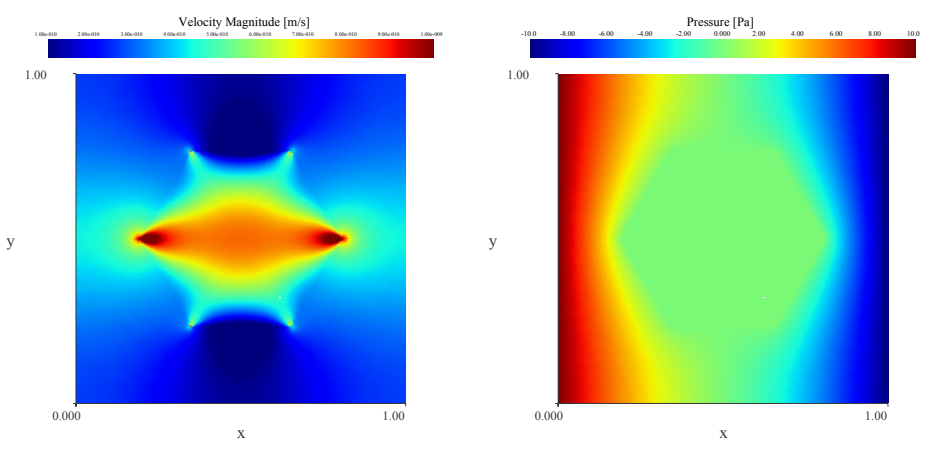

4.8(c): Hexagonal-shaped vug.
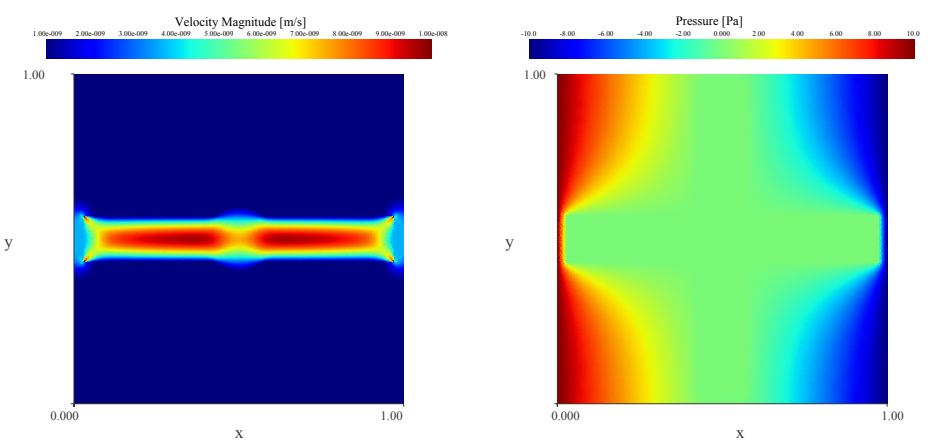

4.8(d): Cross-shaped vug.

Figure 4.8: Pressure and velocity field for periodic cells described in fig. 4.5. The sub-domains area of the periodic cells is constant and set as $A_{\Omega_{D}} / A_{\Omega}=1 / 4$. 


\section{3}

\section{A Porous Medium System}

Three porous medium systems are studied herein, the first with interconnected fractures, the second with isolated vugs and the third with fractureconnected vugs. Figure 4.9 summarizes the geometric descriptions of the porous medium systems. The system of connected fractures consists of symmetrical fractures of aperture $l / l_{f}=0.5$ communicating all faces of the periodic cell domain. The system of isolated vugs contains two circle-shaped vugs with different radius and a single ellipse-shaped vug. The circle-shaped vugs have $r_{1} / l=0.25$ and $r_{2} / l=0.10$ of radius with its center respectively located at $(0.25,0.75)$ and $(0.25,0.25)$. The ellipse-shaped vug has the minor and major axes sizes of $2 a$ and $2 b$ and the center located at $(0.75,0.50)$. The system of connected vugs is simply the superposition of the isolated vugs and connected fractures system.

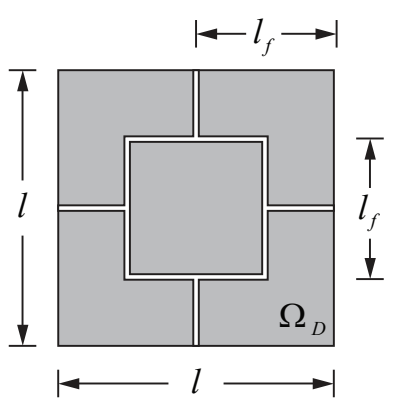

4.9(a): System of connected fractures.

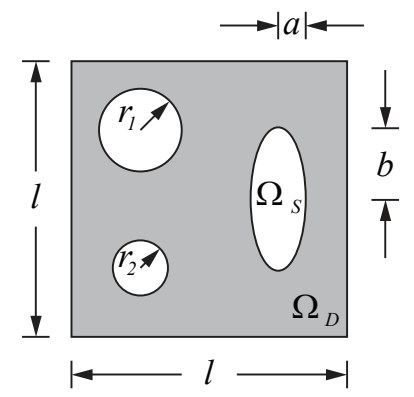

4.9(b): System of isolated vugs.

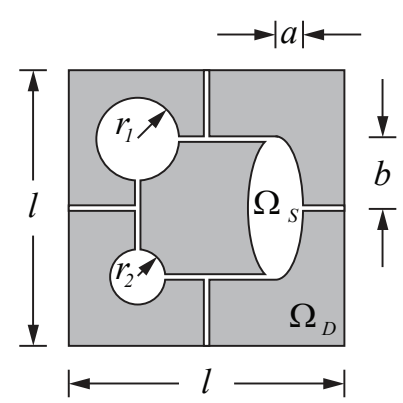

$4.9(\mathrm{c})$ : System of fractureconnected vugs.

Figure 4.9: Porous medium system with interconnected fractures, isolated vugs and fracture-connected vugs. The periodic cell size is $l$, the $\Omega_{D}$ and $\Omega_{S}$ respectively stands for Darcy and Stokes sub-domains. System of connected fractures with $l / l_{f}=0.5$ and fracture aperture $a / l=0.01$. System of isolated vugs with $r_{1} / l=0.25, r_{2} / l=0.10, a / l=0.25$ and $b / l=0.50$. The system of connected vugs is simply the superposition of the isolated vugs fig. 4.9(b) and connected fractures system fig. 4.9(a).

The system of isolated vugs and fracture-connected vugs presents an asymmetry in its shape due to the position and different size of the vugs. As such a scaled-up permeability tensor is calculated by imposing a pressure gradient on both pairs of opposite faces. The system had results similar those previously shown in sections 4.1 and 4.2. Table 4.5 shows the results for the scaled-up permeability tensor and figs. 4.10 to 4.12 respectively shows the pressure and velocity field for the isolated vugs, connected fractures and fracture-connected vugs system. 
Table 4.5: Estimated permeability tensor for the isolated vugs, connected fractures and fracture-connected vugs system.

\begin{tabular}{rc}
\hline & Estimated permeability tensor $k_{a b s}[\mathrm{mD}]$ \\
\hline Isolated Vugs & {$\left[\begin{array}{cc}1.38 E+01 & 0.00 \\
0.00 & 1.57 E+01\end{array}\right]$} \\
Connected Fracture & {$\left[\begin{array}{cc}2.20 E+10 & 0.00 \\
0.00 & 2.20 E+10 \\
1.92 E+08 & 0.00 \\
0.00 & 1.67 E+08\end{array}\right]$} \\
\hline
\end{tabular}

The connected fractures show an isotropic scaled-up permeability tensor whereas the isolated vugs and fracture-connected vugs do not. The isolated vug system presented an estimated scaled-up permeability of the same order of magnitude as the matrix permeability. The fracture and fracture-connected vug system have permeability in the same order of magnitude as the estimated permeability presented at section 4.1 .

Figure 4.10 shows the pressure and velocity field for the simulated cases imposing flow in the $x$ - and $y$-direction where it is possible to see the preferential flow path being formed. For the simulated case with flow in the $x$-direction, fig. 4.10(a), the highest velocity is mainly associated to the circular vugs and for the simulated case with flow in the $y$-direction, fig. 4.10(b), the overall velocity magnitude increases and is mainly concentrated towards the ellipsoid vug.

As the fracture system is perfectly symmetric, only the case simulated imposing flow at $x$-direction is shown in fig. 4.11 . 

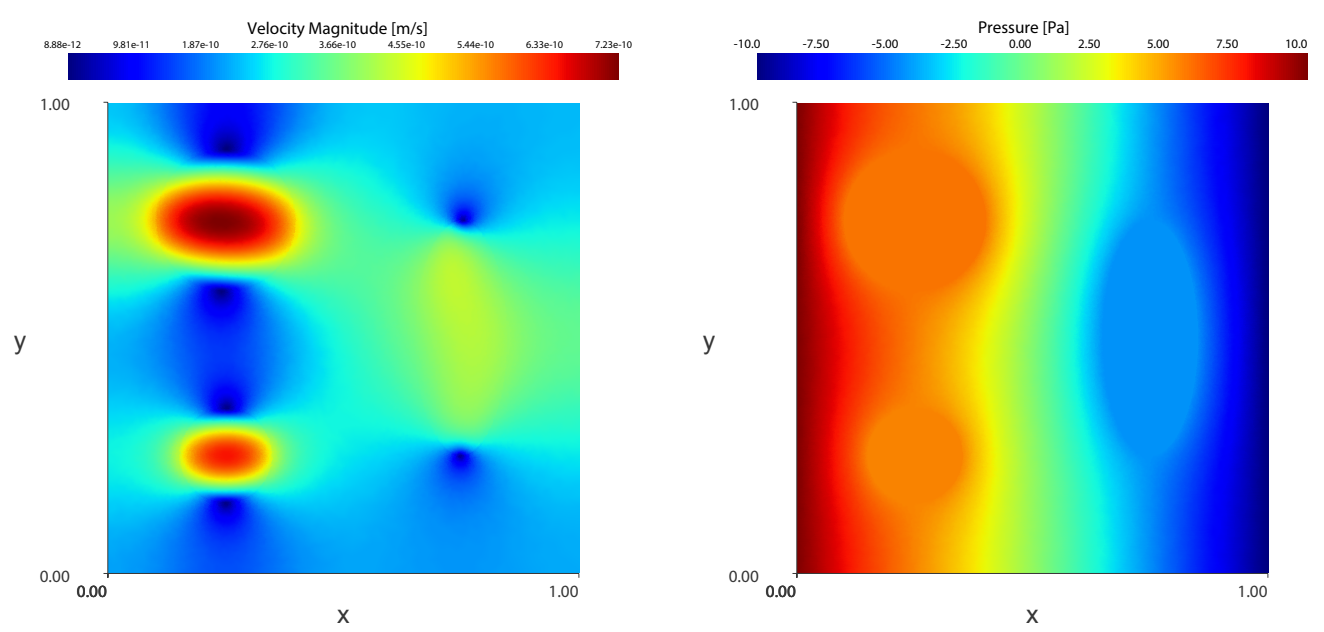

4.10(a): Velocity and pressure distribution for $x$-direction flow.
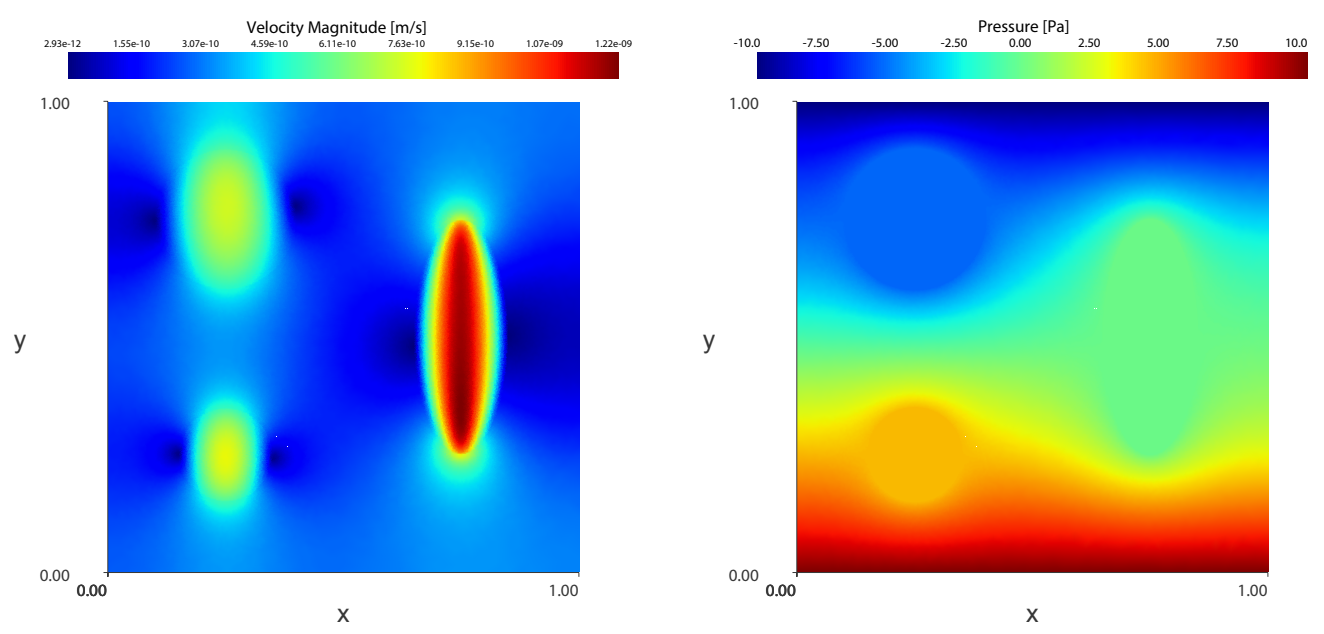

4.10(b): Velocity and pressure distribution for $y$-direction flow.

Figure 4.10: Velocity magnitude and pressure distribution for the system of isolated inclusions described at 4.9(b). 

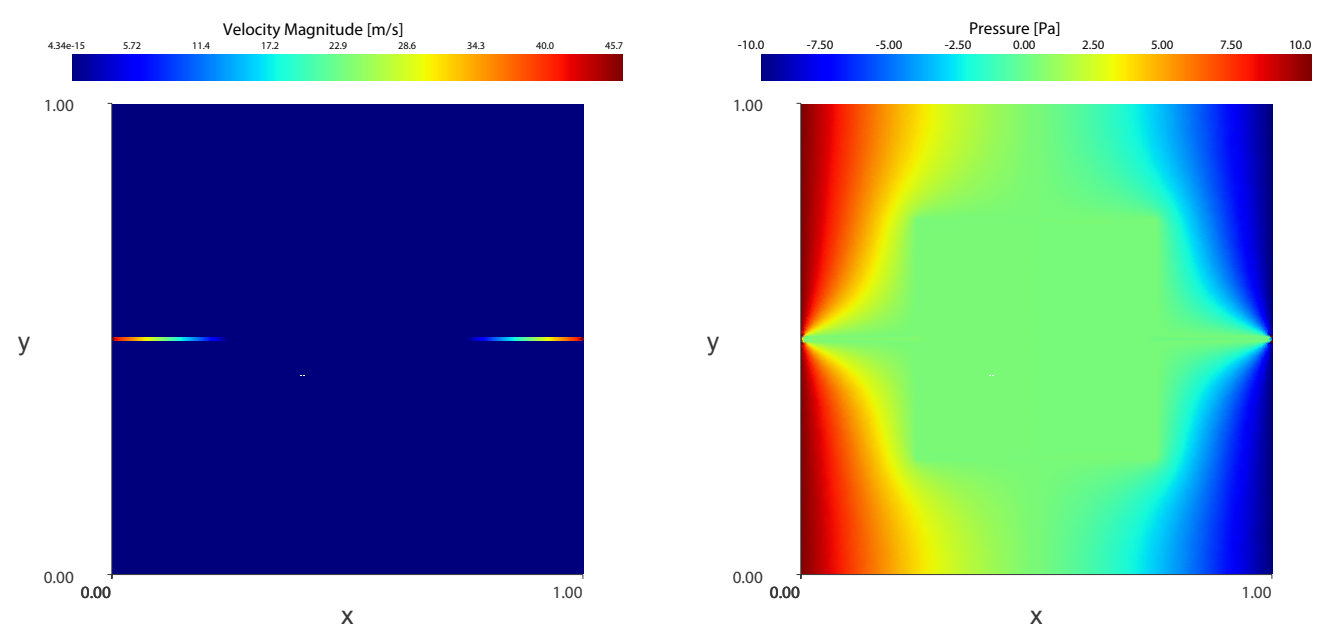

Figure 4.11: Velocity magnitude and pressure distribution for the system of connected fractures described at fig. 4.9(a). 

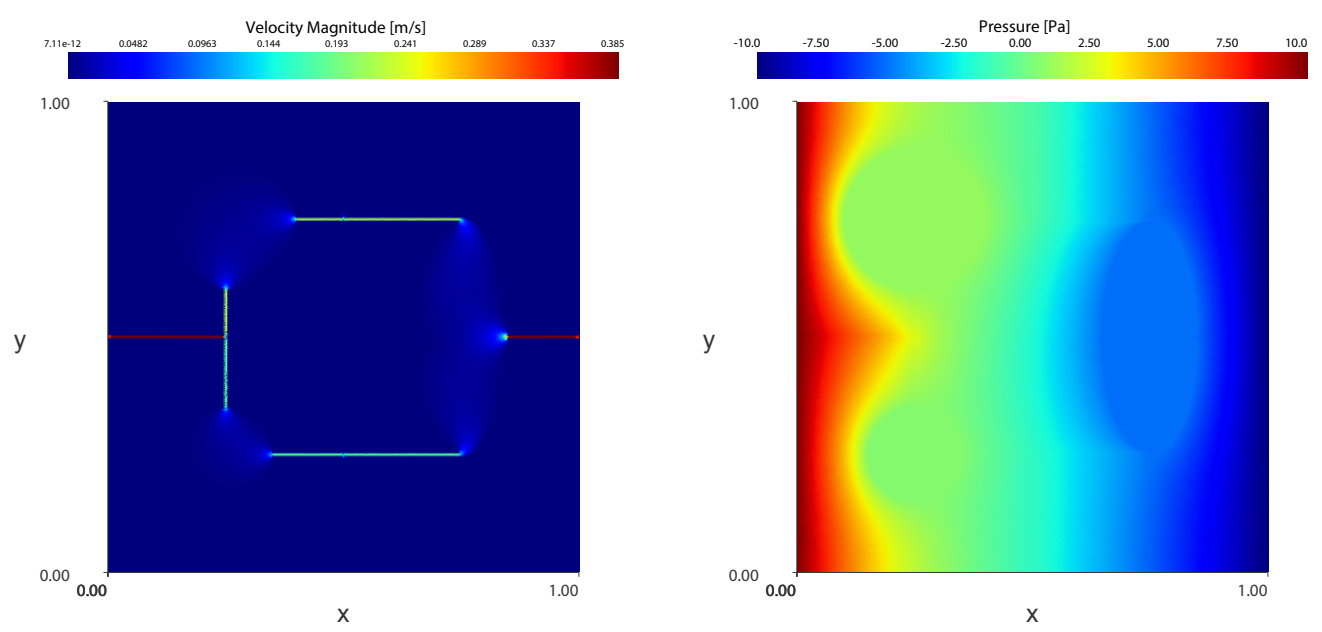

4.12(a): Pressure distribution for $x$-direction flow.
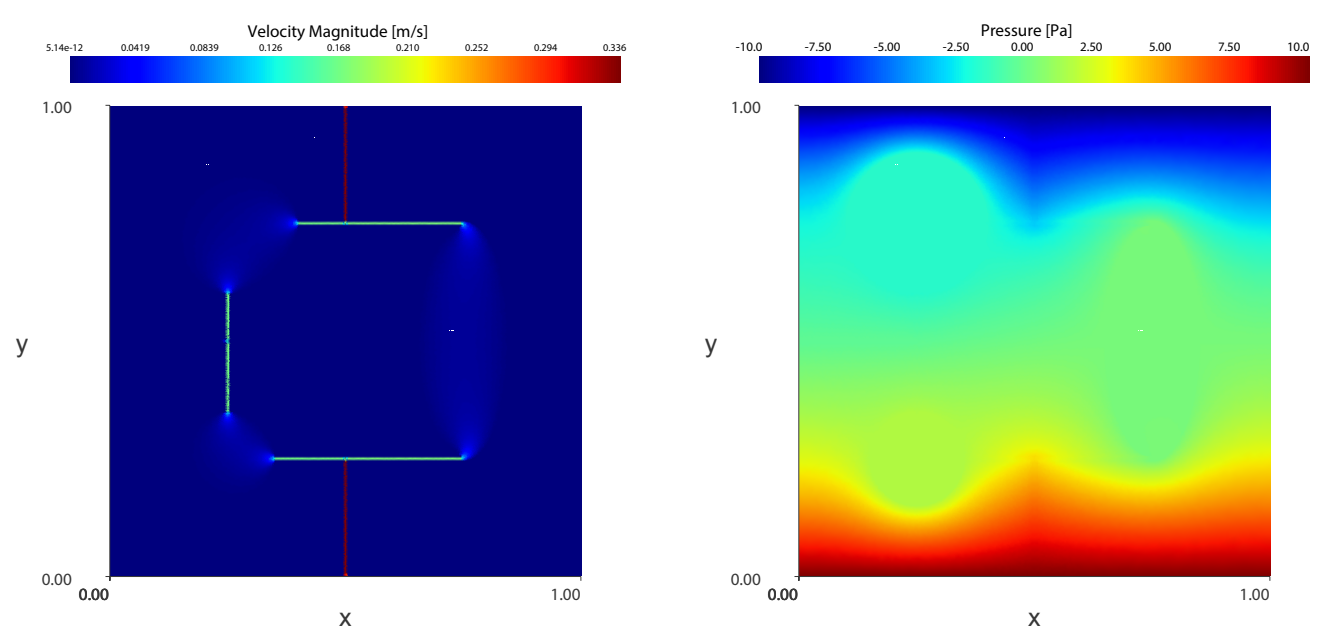

4.12(b): Velocity magnitude for $y$-direction flow.

Figure 4.12: Velocity magnitude and pressure distribution for the system of connected inclusions described at 4.9(c). 


\section{4}

\section{Core Samples}

The developed methodology is applied for permeability scaling-up of two rock samples. Both core samples were evaluated and characterized using a conventional $\mu$-Tomography $(\mu \mathrm{CT})$ acquisition. The $\mu \mathrm{CT}$ provides a twoor three-dimensional high resolution description of pore structure. The pore space and inter-connectivity is accurately characterized and modeled for later permeability scaling-up.

\subsection{1}

\section{An Austin Chalk Sample}

The Austin Chalk sample consists of a limestone composed of ooids and fossils grains. Table 4.6 contains a summary of the plug geometry details and the Routine Core Analysis (RCA) results. The sample has a diameter of 1.35 $\mathrm{cm}$, volume of $14.02 \mathrm{~cm}^{3}$, grain density of $2.72 \mathrm{~g} / \mathrm{cm}^{3}$, measured porosity of $22.74 \%$ and permeability of $11.00 \mathrm{mD}$.

Table 4.6: Core plug geometry details and routine core analysis results for the Austin Chalk sample.

\begin{tabular}{rrrc}
\hline \multicolumn{2}{c}{ Plug Geometry } & \multicolumn{2}{c}{ Routine Core Analysis } \\
\hline Diameter $[\mathrm{cm}]$ & 0.80 & Permeabilty $[\mathrm{mD}]$ & 11.00 \\
Length $[\mathrm{cm}]$ & 1.80 & Porosity $[\%]$ & 22.74 \\
Volume $\left[\mathrm{cm}^{3}\right]$ & 3.62 & Grain Density $\left[\mathrm{g} / \mathrm{cm}^{3}\right]$ & 2.72 \\
\hline
\end{tabular}

The sample was submitted to a $\mu \mathrm{CT}$ acquisition performed at Schlumberger Moscow Research Center (SMR). The source of the $\mu \mathrm{CT}$ had $100 \mathrm{eV}$ at $100 \mu \mathrm{A}$ resulting in a resolution of $2.32 \mu \mathrm{m} / \mathrm{px}$. There were 4650 slices containing $4000 \times 4000$ pixels each. Figure 4.13 shows the $\mu \mathrm{CT}$ slice and the cropped area that will be used herein. The $\mu \mathrm{CT}$ slices consists of an attenuation intensity map that was reworked into a grayscale image. This image was further processed using a five-point median filter in order to reduce the noise.

Figure 4.13 is possible to see ooid-shaped structures that has an ellipsoid format with edges darker than the surrounding and interior material. The overall structure of the ooids and the presence of darker regions on the edges of the structure indicates materials with resolution lower than the $\mu \mathrm{CT}$ resolution. The image was segmented into three regions, one containing the vugs and two containing porous matrix with different properties. The segmentation was accomplished using two fixed cut-offs, 0.40 and 0.80. Everything below 0.40 is modeled as Stokes region $\Omega_{S}$, between 0.40 and 0.80 as Darcy region 


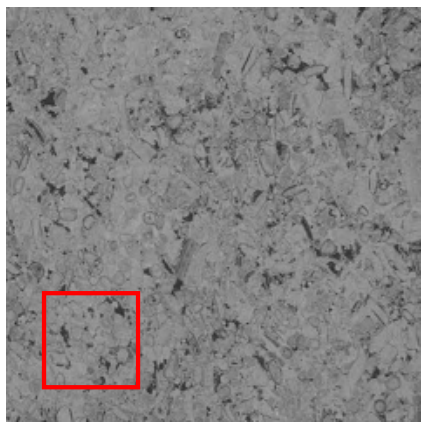

4.13(a): $\mu \mathrm{CT}$ slice.

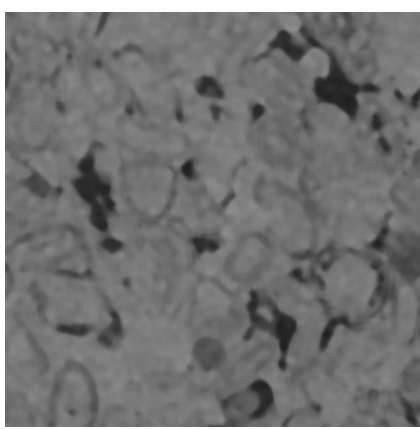

4.13(b): $\mu \mathrm{CT}$ detail.

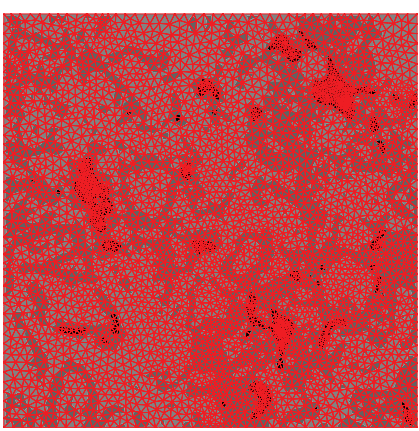

4.13(c): $\mu \mathrm{CT}$ mesh.

Figure 4.13: Micro-tomography $(\mu \mathrm{CT})$ slice from an Austin Chalk sample. The red square in fig. 4.13(a) delimitates the studied region and its displayed at fig. 4.13(b). Figure 4.13(c) shows the numerical mesh in red overlapping regions with discrete properties.

$\Omega_{D 1}$ with $k_{1}=1000 \mathrm{mD}$ and above 0.80 as Darcy region $\Omega_{D 2}$ with $k_{2}=10 \mathrm{mD}$. Figure 4.13(c) contains the finite element grid overlapping the segmented regions. The finite element grid is refined accordingly to the grayscale intensity, as darker finer the grid.

The scaled-up permeability tensor was estimated similarly to the other asymmetric cases, aligning pressure boundary conditions to both pair of opposite faces and back-calculating the permeability for each case. The scaledup permeability tensor can be seen in table 4.7. The sample segmented region doesn't present any preferential vug arrangement and the ooids are uniformly distributed, thus the fairly equal components of the scaled-up permeability tensor.

Figure 4.14 shows the results for the pressure and velocity field of the Austin Chalk sample. The pressure distribution for the simulated case imposing flow in the $x$-direction, fig. 4.14(a), shows an uniform gradient and for the $y$-direction case, fig. 4.14(b), a steeper gradient. The velocity magnitude shows a preferential path aligned with the vugs that branches along with its distribution.

Table 4.7: Estimated permeability tensor for the Austin Chalk analogue sample.

Austin Chalk sample $k_{a b s}[\mathrm{mD}] \quad\left[\begin{array}{cc}1.98 E+02 & 0.00 \\ 0.00 & 1.90 E+02\end{array}\right]$



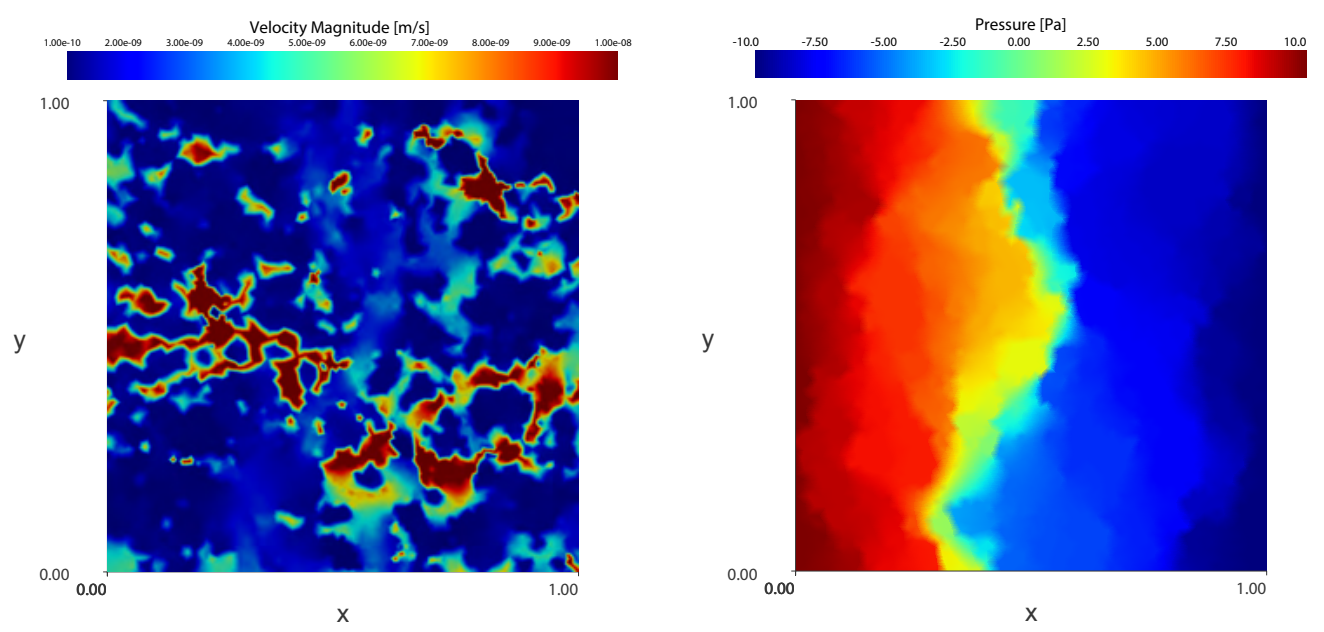

4.14(a): Velocity and pressure for $x$-direction.
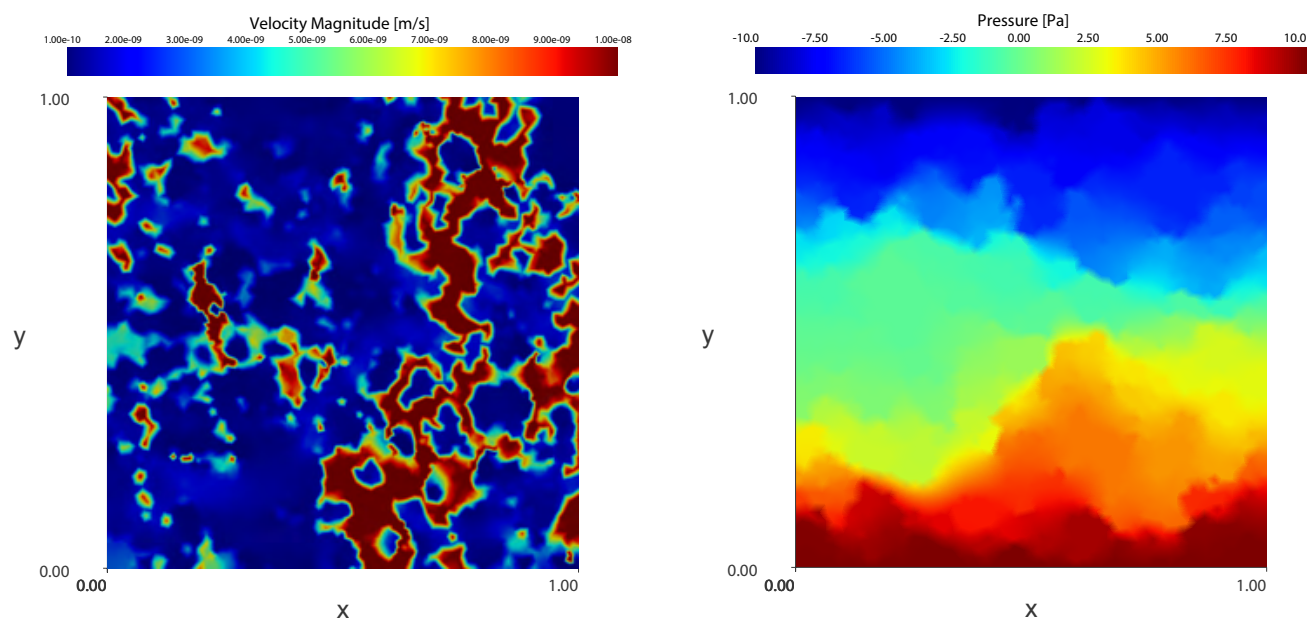

4.14(b): Velocity and pressure for $y$-direction.

Figure 4.14: Velocity magnitude and pressure distribution for the microtomography slice of Austin Chalk sample shown at 4.13.

\subsection{2}

\section{A Coquinas Analogue Sample}

The Coquinas Analogue sample consists of a limestone composed of bivalves shell fragments, commonly denominated as coquinas. Table 4.8 contains a summary of the plug geometry details and the RCA results. The sample has a diameter of $2.54 \mathrm{~cm}$, volume of $40.08 \mathrm{~cm}^{3}$, grain density of $2.71 \mathrm{~g} / \mathrm{cm}^{3}$, measured porosity of $21.5 \%$ and permeability of $358 \mathrm{mD}$.

The sample was submitted to a $\mu$-Tomography $(\mu \mathrm{CT})$ acquisition performed at the Nuclear Instrumentation Laboratory (LNI) at the Federal University of Rio de Janeiro (UFRJ). The source of the $\mu \mathrm{CT}$ had $130 \mathrm{eV}$ at 61 $\mu \mathrm{A}$ resulting in a resolution of $20 \mu \mathrm{m} / \mathrm{px}$. There were 4235 slices containing 
Table 4.8: Core plug geometry details and routine core analysis results for the Coquinas sample.

\begin{tabular}{rcrc}
\hline \multicolumn{2}{c}{ Plug Geometry } & \multicolumn{2}{c}{ Routine Core Analysis } \\
\hline Diameter $[\mathrm{cm}]$ & 2.54 & Permeabilty $[\mathrm{mD}]$ & 358.00 \\
Length $[\mathrm{cm}]$ & 7.91 & Porosity $[\%]$ & 21.50 \\
Volume $\left[\mathrm{cm}^{3}\right]$ & 40.08 & Grain Density $\left[\mathrm{g} / \mathrm{cm}^{3}\right]$ & 2.71 \\
\hline
\end{tabular}

$1420 \times 1420$ pixels each. Figure 4.15 shows the $\mu \mathrm{CT}$ slice and the cropped area that will be used herein. The $\mu \mathrm{CT}$ slices consists of an attenuation intensity map that was reworked into a grayscale image. This image was further processed using a five-point median filter in order to reduce the noise.

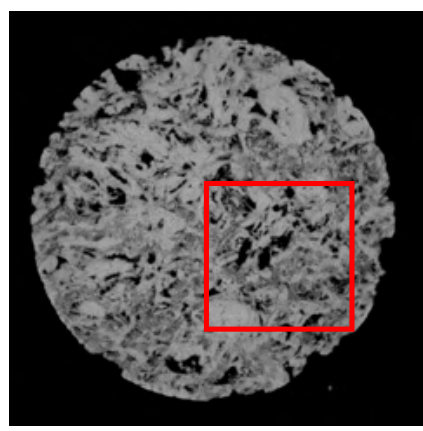

4.15(a): $\mu \mathrm{CT}$ slice.

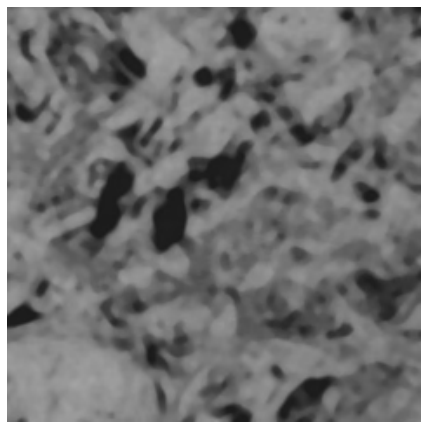

4.15(b): $\mu \mathrm{CT}$ detail.

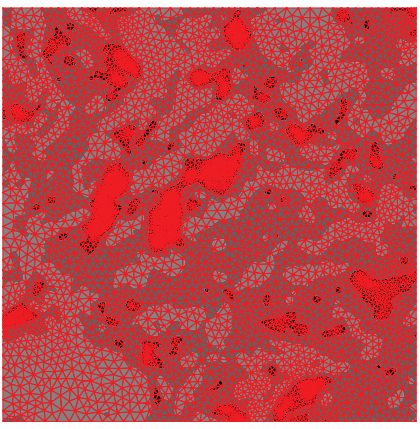

4.15(c): $\mu \mathrm{CT}$ mesh.

Figure 4.15: Micro-tomography ( $\mu \mathrm{CT}$ ) slice from a Coquinas analogue sample. The red square in fig. 4.15(a) delimitates the studied region and its displayed at fig. 4.15(b). Figure 4.15(c) shows the numerical mesh in red overlapping regions with discrete properties.

Figure 4.15 is possible to see structures formed by the shell fragments that has a clear concave format with darker interior material. The image was segmented into three regions, one containing the vugs and two containing porous matrix with different properties. The segmentation was accomplished using two fixed cut-offs, 0.275 and 0.705 . Anything below 0.275 is modeled as Stokes region $\Omega_{S}$, between 0.275 and 0.705 as Darcy region $\Omega_{D 1}$ with $k_{1}=1000$ $\mathrm{mD}$ and above 0.705 as Darcy region $\Omega_{D 2}$ with $k_{2}=10 \mathrm{mD}$. Figure $4.15(\mathrm{c})$ contains the finite element grid overlapping the segmented regions. The finite element grid is refined accordingly to the grayscale intensity, as darker finer the grid.

The scaled-up permeability tensor was estimated similarly to the other asymmetric cases, aligning pressure boundary conditions to both pair of opposite faces and back-calculating the permeability for each case. The permeability tensor can be seen in table 4.9. The segmented sample presents a preferential 
vug arrangement in the $y$-direction simulated case, thus $k_{y y}$ being slightly bigger than $k_{x x}$.

Table 4.9: Estimated permeability tensor for the Coquinas analogue sample.

Coquinas sample $k_{a b s}[\mathrm{mD}] \quad\left[\begin{array}{cc}1.48 E+02 & 0.00 \\ 0.00 & 2.57 E+02\end{array}\right]$

Figure 4.16 shows the results for the pressure and velocity field of the Coquinas sample. Both the pressure distribution for the $x$-direction simulated case, fig. 4.16(a), and for the $y$-direction simulated case, fig. 4.16(b), shows a steep and irregular gradient. The velocity magnitudes, clearly show preferential paths aligned with the vugs distribution and connectivity.
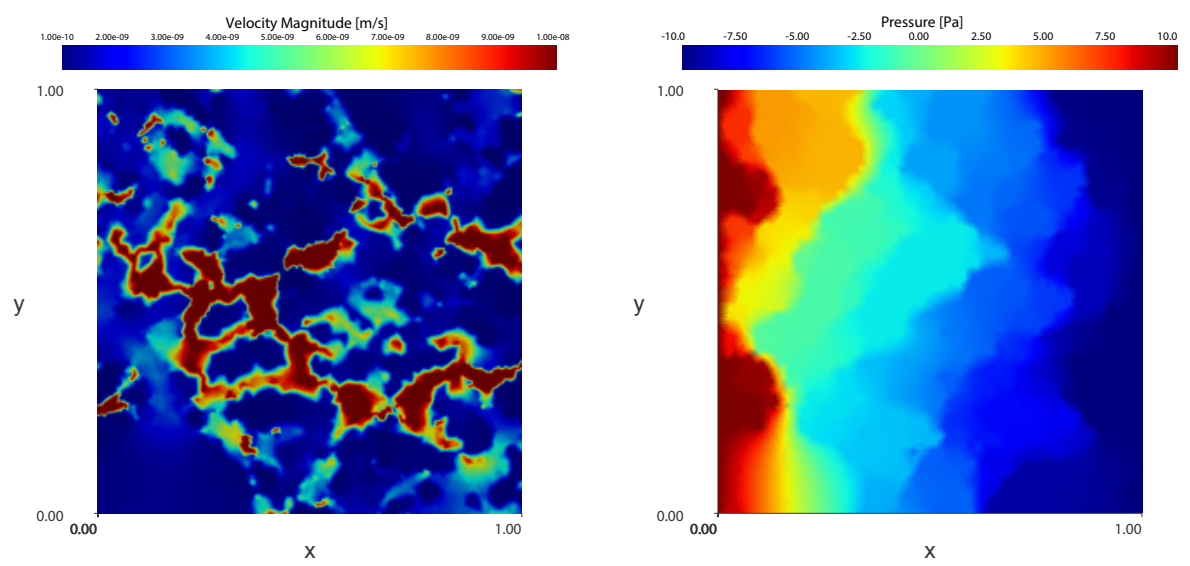

4.16(a): Velocity and pressure for $x$-direction.
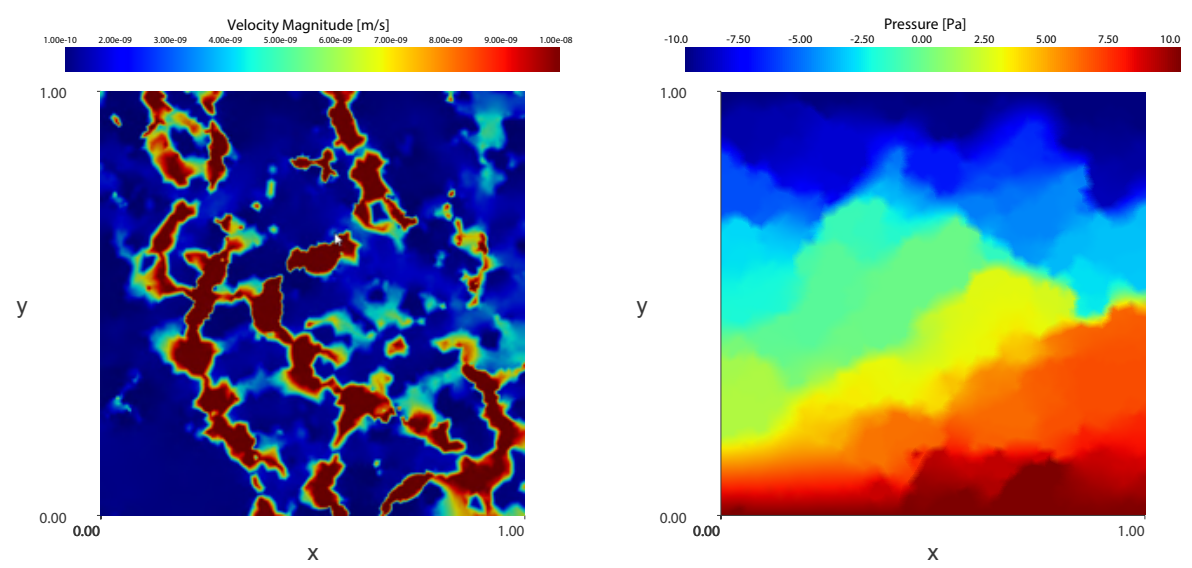

4.16(b): Velocity and pressure for $y$-direction.

Figure 4.16: Velocity magnitude and pressure distribution for the microtomography slice of Coquinas analogue sample shown at 4.15. 


\section{5 \\ Representative Elementary Study}

A REA size study was conducted using the same $\mu$-slice presented in fig. 4.15. The sizes selected are present in table 4.10, in summary it ranges from $0.0002 m$ to $0.008 m$ with 5460 extracted sub-samples. Figure 4.17 illustrates the sample partitioning and number of sub-samples created and table 4.11 contains a basic set of statistical indicators for the estimated permeability values. Both $x$ - and $y$-direction simulated cases presents a similar trend and do not significantly differ from each other, as seen in table 4.9.

Figures 4.18 and 4.19 respectively shows REA results for the estimated scaled-up permeability distribution for $x$ - and $y$-direction cases. The color of the points gets darker as more points occupies the same region. There are two different clusters, one averaging at $10^{13} \mathrm{mD}$ and the other averaging $10^{2}$ to $10^{3} \mathrm{mD}$. The cluster formed at the top of the chart has selected sub-samples and highlights the channels that are formed communicating opposite faces of the REA. For both simulated cases directions it is possible to observe how the channel narrows as the REA length increases and the cluster vanishes for $l>0.1 \mathrm{~cm}$.

Figures 4.20 and 4.21 shows the results of the previously described charts filtered out the permeability values averaging over $10^{8} \mathrm{mD}$ to keep the chart concise and remove bias out of the calculated statistics. The darker blue shade corresponds to the first standard deviation from the average and the lighter blue shade the second standard deviation from the average. The blue line correspond the the average estimated permeability. As previously shown in the table 4.11 the permeability for the $x$ - and $y$-directions are respectively 297 $\mathrm{mD}$ and $263 \mathrm{mD}$.

Observing the asymptotic trend developed in figs. 4.20 and 4.21 is possible to evaluate the minimum size of the REA. Figures 4.22 and 4.23 show the minimum size estimation for $x$ - and $y$-direction, using the first standard deviation $\sigma$, blue dots, and the laboratory measured permeability of the sample, blue line. There were a small difference between the estimated size for each direction, for the $x$-direction the minimum size was estimated as $l=1.27 \mathrm{~cm}$ and for the $y$-direction as $l=1.59 \mathrm{~cm}$. 


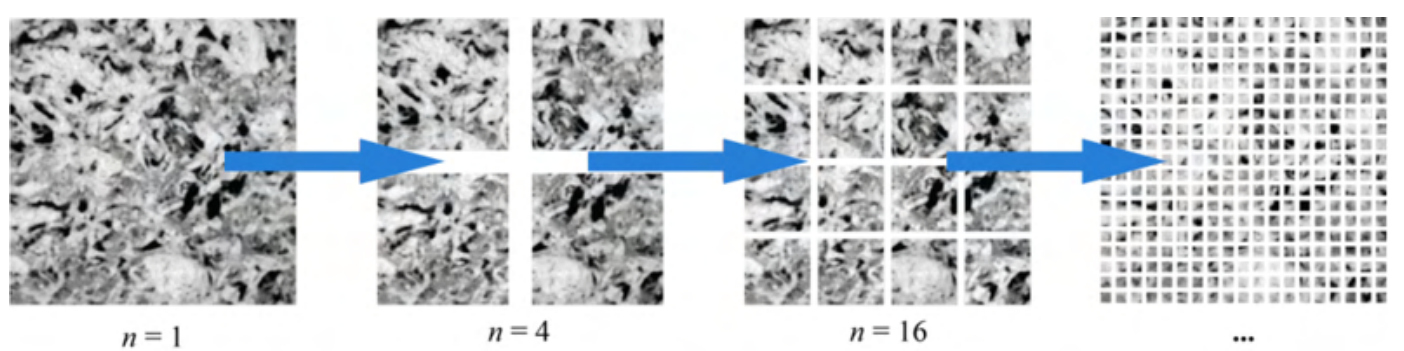

Figure 4.17: Representative Elementary Area (REA) size partitioning and number of sub-samples.

Table 4.10: Representative Elementary Area (REA) length sizes and number of selected sub-samples.

\begin{tabular}{ccc}
\hline$L / l$ & REA size $[\mathbf{m}]$ & N. Sub-Samples \\
\hline $1 / 400$ & $8.13 \mathrm{E}-03$ & 4 \\
$1 / 200$ & $4.06 \mathrm{E}-03$ & 16 \\
$1 / 100$ & $2.03 \mathrm{E}-03$ & 64 \\
$1 / 50$ & $1.02 \mathrm{E}-03$ & 256 \\
$1 / 25$ & $5.08 \mathrm{E}-04$ & 1024 \\
$1 / 10$ & $2.03 \mathrm{E}-04$ & 4096 \\
\hline
\end{tabular}

Table 4.11: Representative Elementary Area (REA) statistics for the estimated permeability in the $x$ - and $y$-direction.

\begin{tabular}{ccccccc}
\hline & \multicolumn{3}{c}{ Permeability x-direction } & \multicolumn{3}{c}{ Permeability y-direction } \\
L/1 & min. & mean & max. & min. & mean & max. \\
\hline $1 / 400$ & $1.63 \mathrm{E}+00$ & $2.97 \mathrm{E}+00$ & $4.16 \mathrm{E}+00$ & $1.28 \mathrm{E}+00$ & $2.63 \mathrm{E}+00$ & $5.62 \mathrm{E}+00$ \\
$1 / 200$ & $2.27 \mathrm{E}-01$ & $1.19 \mathrm{E}+00$ & $2.32 \mathrm{E}+00$ & $1.73 \mathrm{E}-01$ & $1.08 \mathrm{E}+00$ & $3.21 \mathrm{E}+00$ \\
$1 / 100$ & $8.21 \mathrm{E}-03$ & $4.92 \mathrm{E}-01$ & $3.32 \mathrm{E}+00$ & $1.59 \mathrm{E}-02$ & $4.21 \mathrm{E}-01$ & $3.06 \mathrm{E}+00$ \\
$1 / 50$ & $5.24 \mathrm{E}-03$ & $2.72 \mathrm{E}-01$ & $1.22 \mathrm{E}+01$ & $3.49 \mathrm{E}-03$ & $2.28 \mathrm{E}-01$ & $1.90 \mathrm{E}+01$ \\
$1 / 25$ & $1.04 \mathrm{E}-03$ & $1.70 \mathrm{E}-01$ & $1.80 \mathrm{E}+01$ & $1.05 \mathrm{E}-05$ & $1.36 \mathrm{E}-01$ & $2.49 \mathrm{E}+01$ \\
$1 / 10$ & $9.11 \mathrm{E}-08$ & $9.03 \mathrm{E}-02$ & $2.15 \mathrm{E}+01$ & $2.14 \mathrm{E}+01$ & $4.55 \mathrm{E}-07$ & $8.54 \mathrm{E}-02$ \\
\hline
\end{tabular}




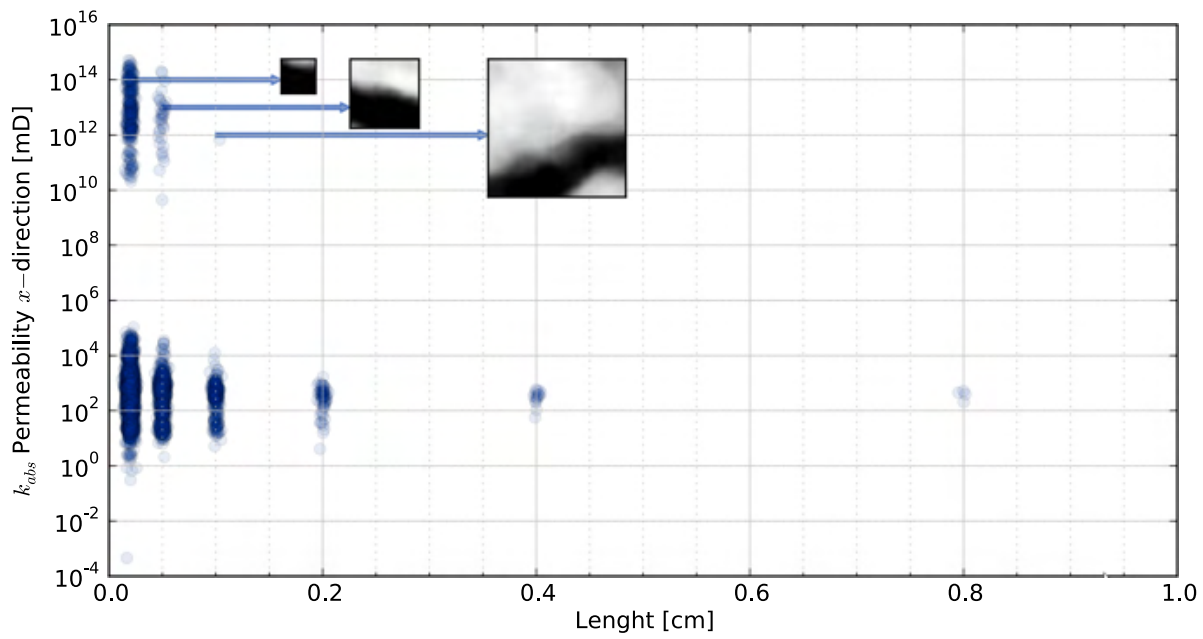

Figure 4.18: Representative Elementary Area (REA) results for the estimated permeability in the $x$-direction. The color of the points gets darker as more points occupies the same region. Sub-samples being displayed at the top exemplifies the channels that communicates opposite faces of the REA.

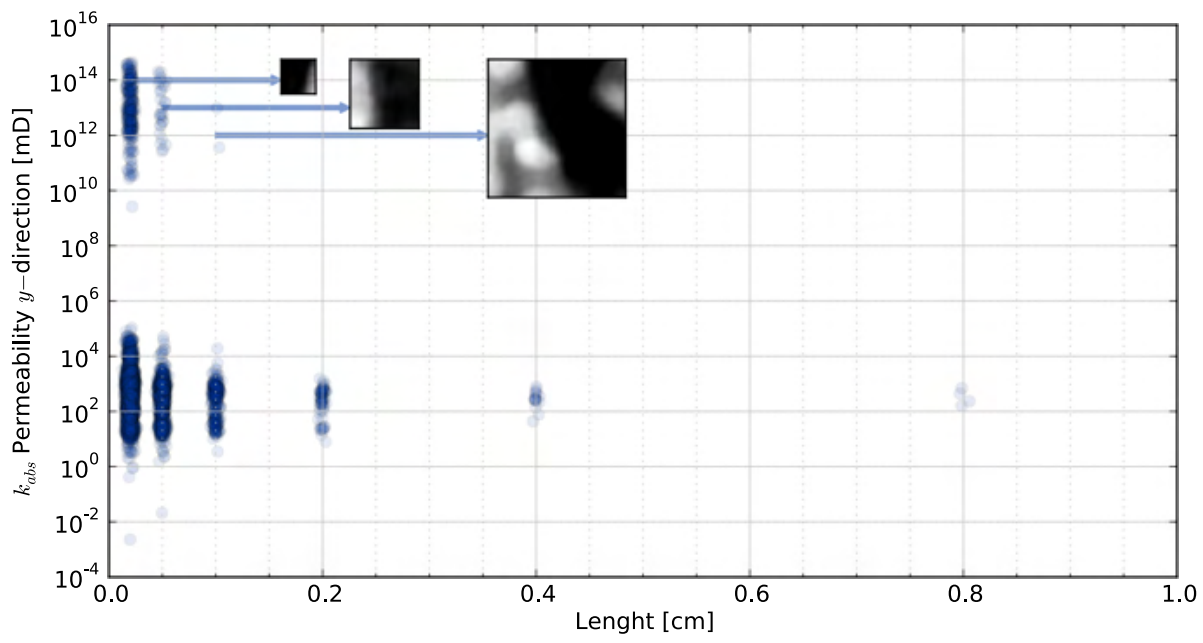

Figure 4.19: Representative Elementary Area (REA) results for the estimated permeability in the $y$-direction. The color of the points gets darker as more points occupies the same region. Sub-samples being displayed at the top exemplifies the channels that communicates opposite faces of the REA. 


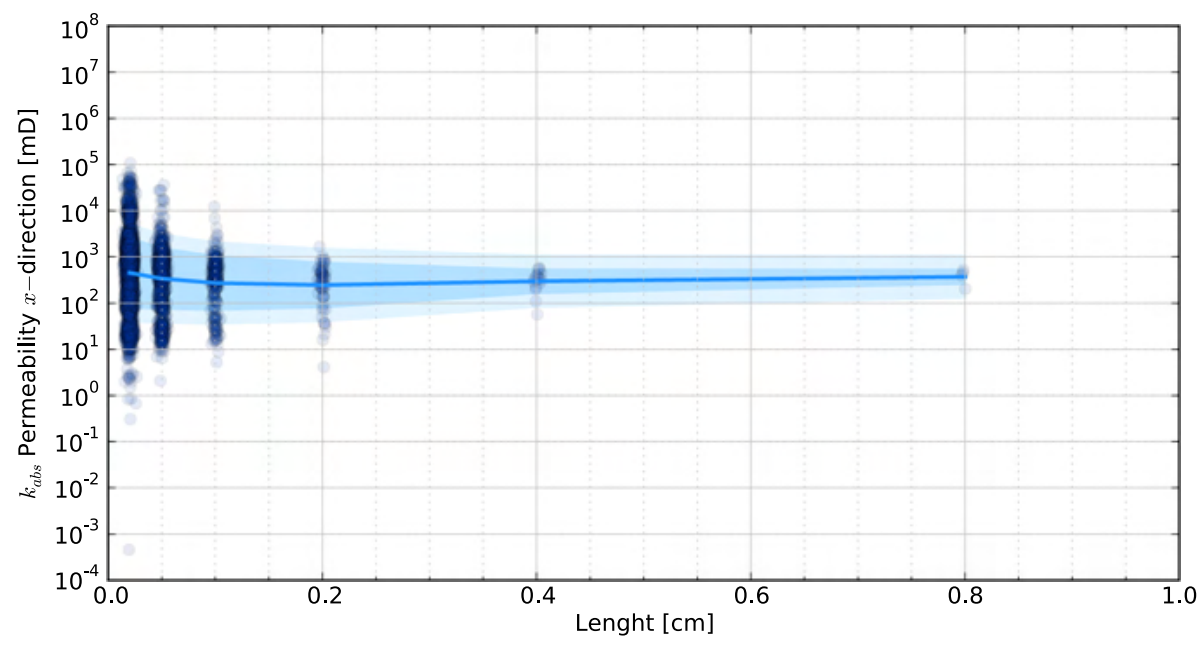

Figure 4.20: Representative Elementary Area (REA) results for the estimated permeability in the $x$-direction with sub-samples with communicated opposite faces filtered. The color of the points gets darker as more points occupies the same region. The darker blue shade filling corresponds to the first standard deviation from the average and the lighter blue shade filling the second standard deviation from the average.

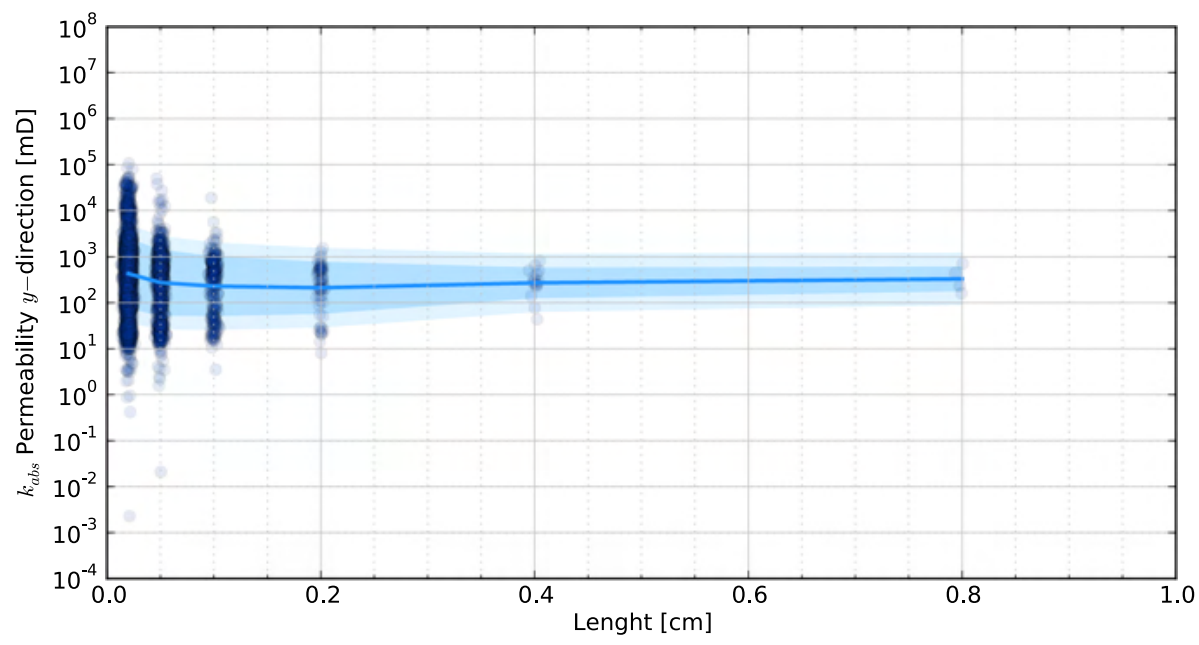

Figure 4.21: Representative Elementary Area (REA) results for the estimated permeability in the $y$-direction with sub-samples with communicated opposite faces filtered. The color of the points gets darker as more points occupies the same region. The darker blue shade filling corresponds to the first standard deviation from the average and the lighter blue shade filling the second standard deviation from the average. 


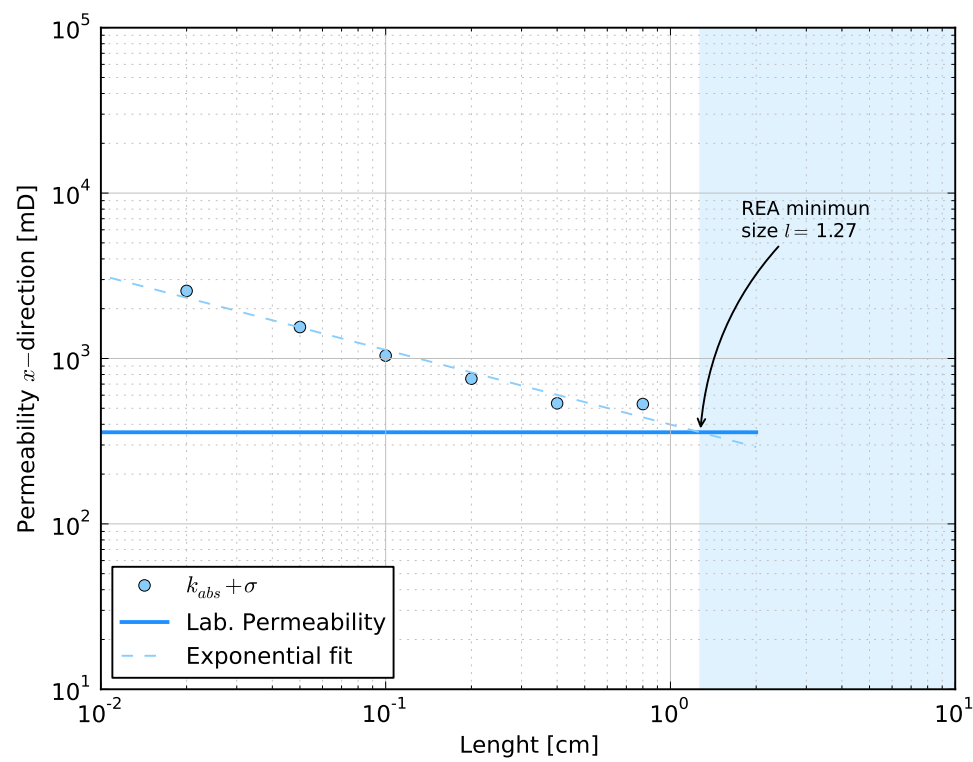

Figure 4.22: Representative Elementary Area (REA) minimum size estimation for $x$-direction, using the first standard deviation $\sigma$, blue dots, and the laboratory measured permeability of the sample, blue line. The estimated REA minimun size was $l=1.27 \mathrm{~cm}$.

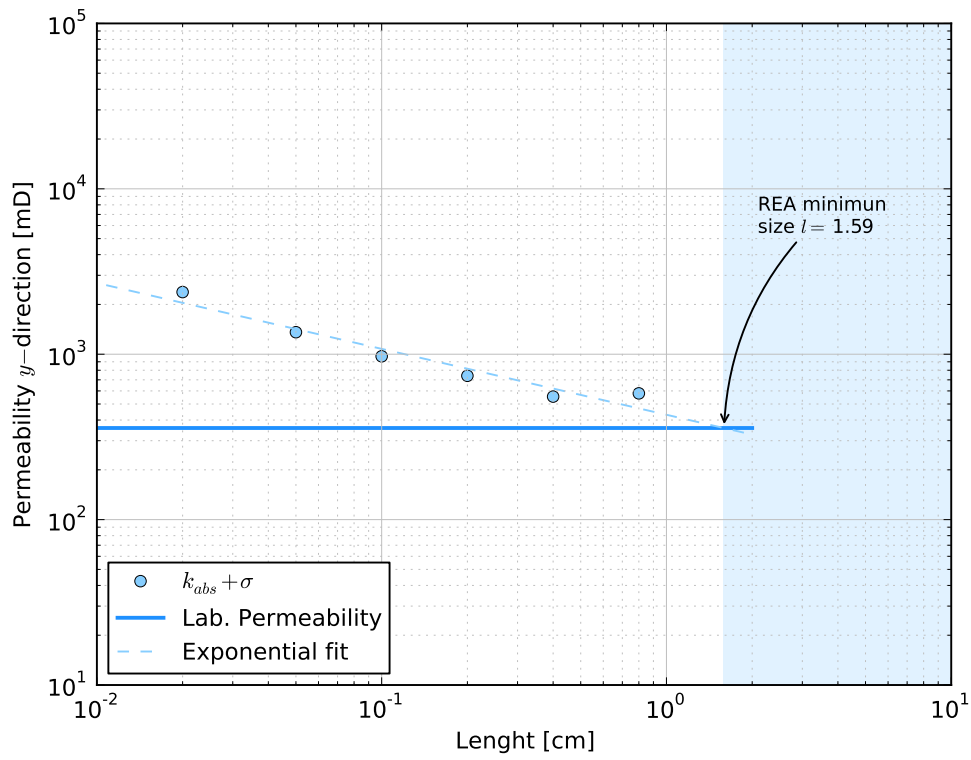

Figure 4.23: Representative Elementary Area (REA) minimum size estimation for $y$-direction, using the first standard deviation $\sigma$, blue dots, and the laboratory measured permeability of the sample, blue line. The estimated REA minimun size was $l=1.59 \mathrm{~cm}$. 


\section{5 \\ Discussions and Conclusions}

The present study had the objective of developing a methodology to evaluate permeability as a scaled-up parameter for heterogeneous porous media. In order to achieve this, Brinkman's flow equation was numerically implemented using the finite element method and a partial differential equation library that is part of the FEniCS project. The study started with a parametric investigation of the presence of vugs and fractures in a porous cell and later applied the methodology to scaling-up permeability tensors of two carbonate rock samples. Finally the results for one of the carbonate rock sample were extended to include a representative elementary area study.

\section{1 \\ Periodic Cells}

The periodic cells elucidated the vug presence and shape effects in the permeability scaling-up results. The presence of channels that connect opposite faces of the periodic cell significantly increase the scaled-up permeability to over 10 to 12 orders of magnitude of what would be expected if no channel was present. The scaled-up permeability is mainly controlled by the aperture of the channel, it rapidly increases for aperture smaller than $1 / 2$ of the periodic cell size and it smoothly increases once its above this value. The effect of the matrix permeability changes is hardly noticed on the scaled-up permeability. Inside the porous domain the velocity behaves accordingly to Darcy's law as if the channel was not present. The shape of the vug starts to affect the scaled-up permeability as the vug shape comes closer to that of connected channels.

Next the presence of a system of fractures or vugs was studied. The presence of vugs with different shapes and sizes resulted in an anisotropic scaled-up permeability. The isolated vugs case had scaled-up permeability of the order of the matrix permeability, with higher the higher component of the permeability tensor aligned with the major axis of the elliptical vug. Both fracture-connected vugs and connected fractures cases had scaled-up permeability orders of magnitude higher than the matrix permeability due to the presence of the connected channels. These results were consistent to the 
periodic cells cases.

\section{2}

\section{Austin Chalk and Coquinas analogue samples}

Following the parametric periodic cell's study, two rock samples were selected to apply the developed methodology. The carbonate rock samples were selected because of the high heterogeneity content and presence of vugs. They were evaluated using a conventional $\mu$-Tomography acquisition and the study used the discrete segmented regions of the sample in order to scaled-up the permeability tensor. The estimated permeability for the Austin Chalk sample was an order of magnitude higher for both components of the symmetric tensor than the measured laboratory permeability. The Coquinas sample had one of the components of the estimated permeability tensor closer to the laboratory measured permeability, but still with significant deviation. There are two sources of possible mistake on the adopted methodology. One arises from the two-dimensional approach that isn't enough in case the poreconnectivity extends beyond the modeled plane. The other source of error arises from macroscopic term of the Brinkman equation. There is a strong assumption for the porous matrix of both samples, in which there should be connected pores and that they present previously known permeability. Ideally it is necessary to either properly characterize the pores in the lowresolution porous matrix and correctly assign a permeability for that region or history match the permeability field so that it is consistent with the laboratory permeability.

\section{3 \\ Representative elementary study}

Despite the presented mismatch, a study was conducted into the area representativeness of the Coquinas analogue sample with the aim of estimating a minimum representative elementary area (REA) size for the sample. The sample was segmented into smaller sub-samples and further was estimated both components of the permeability tensor were estimated for each sub-sample. The results clearly showed that the REA size needs to be bigger than the smaller features present on the sample in such a way that the vugs are confined inside a porous matrix and do not form channels between the sub-samples. The mean value of the estimated permeability for each of the sub-samples asymptotically converges to the sample's previously determined scaled-up permeability. 


\section{Bibliography}

[1] CHOQUETTE, P. W. PRAY, L. C., Geological nomenclature and classification of porosity in sedimentary carbonates, American Association of Petroleum Geologists Bulletin, vol. 54, no. 2, pp. 207-250, 1970.

[2] AHR, W. M., Geology of carbonate reservoirs: The Identification, Description, and Characterization of Hydrocarbon Reservoirs in Carbonate Rocks. John Wiley \& Sons, Inc, 2011.

[3] FOLK, R. L., Geological nomenclature and classification of porosity in sedimentary carbonates, American Association of Petroleum Geologists Bulletin, vol. 43, no. 1, pp. 1-38, 1959.

[4] DUNHAM, R. J., Classification of carbonate rocks according to depositional texture., American Association of Petroleum Geologists Memoir, vol. 1, no. 1, pp. 108-121, 1962.

[5] FANCHI, J., Principles of Applied Reservoir Simulation. Chemical, Petrochemical \& Process, Gulf Professional Publishing, 2006.

[6] ROSA, A. J., DE SOUZA CARVALHO, R., XAVIER, J. A. D., Engenharia de reservatórios de petróleo. Editora Interciência, 2006.

[7] BLUNT, M. J., BIJELJIC, B., DONG, H., GHARBI, O., IGLAUER, S., MOSTAGHIMI, P., PALUSZNY, A., PENTLAND, C., Pore-scale imaging and modelling, Advances in Water Resources, vol. 51, no. 0, pp. 197-216, 2013. 35th Year Anniversary Issue.

[8] CHEN, S. DOOLEN, G. D., Lattice Boltzmann method for fluid flows, Annual Review of Fluid Mechanics, vol. 30, no. 1, pp. 329-364, 1998.

[9] NAGEL, W., High Performance Computing in Science and Engineering '08: Transactions of the High Performance Computing Center, Stuttgart (HLRS) 2008. Mathematics and Statistics, Springer, 2009. 
[10] FERRÉOL, B. ROTHMAN, D. H., Lattice-Boltzmann simulations of flow through Fontainebleau sandstone, Transport in Porous Media, vol. 20, no. 1-2, pp. 3-20, 1995.

[11] FATT, I., The network model of porous media - i. capillary pressure characteristics, AIME Petroleum Transactions, vol. 207, no. 59, pp. 144-181, 1957.

[12] BLUNT, M. J., JACKSON, M. D., PIRI, M., VALVATNE, P. H., Detailed physics, predictive capabilities and macroscopic consequences for pore-network models of multiphase flow, Advances in Water Resources, vol. 25, no. 8-12, pp. 1069-1089, 2002.

[13] DEMIANOV, A., DINARIEV, O., EVSEEV, N., Density functional modelling in multiphase compositional hydrodynamics, The Canadian Journal of Chemical Engineering, vol. 89, no. 2, pp. 206-226, 2011.

[14] KOROTEEV, D., DINARIEV, O., EVSEEV, N., KLEMIN, D., NADEEV, A., SAFONOV, S., GURPINAR, O., BERG, S., VAN KRUIJSDIJK, C., ARMSTRONG, R., MYERS, M. T., HATHON, L., DE JONG, H., Direct hydrodynamic simulation of multiphase flow in porous rock, Proceedings of International Society of Core Analysts Symposium, September 2013.

[15] MOSTAGHIMI, P., BLUNT, M. J., BIJELJIC, B., Computations of absolute permeability on micro-ct images, Mathematical Geosciences, vol. 45, no. 1, pp. 103-125, 2013.

[16] ARBOGAST, T. GOMEZ, M., A discretization and multigrid solver for a darcy-stokes system of three dimensional vuggy porous media, Computational Geosciences, vol. 13, no. 3, pp. 331-348, 2009.

[17] HUANG, Z., YAO, J., LI, Y., WANG, C., LV, X., Numerical calculation of equivalent permeability tensor for fractured vuggy porous media based on homogenization theory, Communications in Computational Physics, vol. 9, no. 1, pp. 180-204, 2011.

[18] POPOV, P., EFENDIEV, Y., QIN, G., Multiscale modeling and simulations of flows in naturally fractured karst reservoirs, Communications in Computational Physics, vol. 6, no. 1, pp. 162-184, 2009.

[19] BACHMAT, Y. BEAR, J., On the concept and size of a representative elementary volume (REV), in Advances in Transport Phenomena in 
Porous Media (Bear, J. Corapcioglu, M. Y., eds.), vol. 128 of NATO ASI Series, pp. 3-20, Springer Netherlands, 1987.

[20] DRUGAN, W. J. WILLIS, J. R., A micromechanics-based nonlocal constitutive equation and estimates of representative volume element size for elastic composites, Journal of the Mechanics and Physics of Solids, vol. 44, no. 4, pp. 497-524, 1996.

[21] KANIT, T., FOREST, S., GALLIET, I., MOUNOURY, V., JEULIN, D., Determination of the size of the representative volume element for random composites: statistical and numerical approach, International Journal of Solids and Structures, vol. 40, no. 13-14, pp. 3647-3679, 2003.

[22] NORDAHL, K. RINGROSE, P., Identifying the representative elementary volume for permeability in heterolithic deposits using numerical rock models, Mathematical Geosciences, vol. 40, no. 7, pp. 753-771, 2008.

[23] WHITE, F. M., Fluid Mechanics. McGraw-Hill international editions, McGraw-Hill, 2003.

[24] CRITTENDEN, J. C., TRUSSElL, R. R., HAND, D. W., HOWE, K. J., TCHOBAnOglous, G., Appendix C: Physical Properties of Water, pp. 1861-1862. John Wiley \& Sons, Inc., 2012.

[25] BATCHELOR, G. K., An Introduction to Fluid Dynamics. Cambridge Mathematical Library, Cambridge University Press, 2000.

[26] SJOBLOM, J., Encyclopedic Handbook of Emulsion Technology. Taylor \& Francis, 2001.

[27] JOLLEY, S. J., Reservoir Compartmentalization. Geological Society special publication, Geological Society, 2010.

[28] HAN, D., NUR, A., MORGAN, D., Effects of porosity and clay content on wave velocities in sandstones, Geophysics, vol. 51, no. 11, pp. 20932107, 1986.

[29] DARCY, H., Les fontaines publiques de la Ville de Dijon, 1856. Bibliothéque Nationale de France, Département Littérature et art, V-13914.

[30] OLAUSSEN, S., BECK, L., FALT, L. M., GRAUE, E., JACOBSEN, K. G., MALM, O. A., SOUTH, D., Gullfaks Field-Norway East Shetland Basin, 
Northern North Sea, pp. 55-83. Treatise Atlas, American Association of Petroleum Geologists, 1992.

[31] LANDAU, L. D. LIFSHITZ, E. M., Fluid Mechanics. Course of theoretical physics V.6, Butterworth-Heinemann Limited, 1987.

[32] WHITAKER, S., Flow in porous media i: A theoretical derivation of Darcy's law, Transport in Porous Media, vol. 1, no. 1, pp. 3-25, 1986.

[33] BRINKMAN, H., On the permeability of media consisting of closely packed porous particles, Applied Scientific Research, vol. 1, no. 1, pp. 8186, 1949.

[34] HUGHES, T. J. R., The finite element method: linear static and dynamic finite element analysis. Dover Civil and Mechanical Engineering Series, Dover Publications, 2000.

[35] LOGG, A., MARDAL, K.-A., WELLS, G. N., OTHERS,, Automated Solution of Differential Equations by the Finite Element Method. Springer, 2012.

[36] LOGG, A. WELLS, G. N., DOLFIN: Automated finite element computing, ACM Transactions on Mathematical Software, vol. 37, no. 2, 2010.

[37] ALN/ES, M. S., LOGG, A., ØLGAARD, K. B., ROGNES, M. E., WELLS, G. N., Unified form language: A domain-specific language for weak formulations of partial differential equations, ACM Transactions on Mathematical Software, vol. To appear, 2013.

[38] KIRBY, R. C. LOGG, A., A compiler for variational forms, ACM Transactions on Mathematical Software, vol. 32, no. 3, 2006.

[39] ALNÆS, M. S., LOGG, A., MARDAL, K.-A., SKAVHAUG, O., LANGTANGEN, H. P., Unified framework for finite element assembly, International Journal of Computational Science and Engineering, vol. 4, no. 4, pp. 231-244, 2009.

[40] KIRBY, R. C., Algorithm 839: Fiat, a new paradigm for computing finite element basis functions, ACM Transactions on Mathematical Software, vol. 30, no. 4, pp. 502-516, 2004.

[41] SCHROEDER, W., MARTIN, K., LORENSEN, B., Visualization Toolkit: An Object-Oriented Approach to 3D Graphics. Kitware, 2000.

[42] PROJECT, F., “The fenics project." http://fenicsproject.org/, February 2014. 
[43] HANSEN, C. D. JOHNSON, C., Visualization Handbook. Elsevier Science, 2011.

[44] BREZZI, F., JIM, J. D., MARINI, L., Two families of mixed finite elements for second order elliptic problems, Numerische Mathematik, vol. 47, no. 2, pp. 217-235, 1985.

[45] BREZZI, F., HUGHES, T. J. R., MARINI, L. D., MASUD, A., Mixed discontinuous galerkin methods for darcy flow, Journal of Scientific Computing, vol. 22-23, no. 1-3, pp. 119-145, 2005.

[46] HOOD, P. TAYLOR, C., Numerical solution of the Navier-Stokes equations using the finite element technique, Computational Fluids, vol. 1, no. 1, pp. 1-28, 1973.

[47] FOSSUM, G. A., HAUGE, V. L., LIE, K.-A., A multiscale mixed finite element method for vuggy and naturally fractured reservoirs, SPE Journal, vol. 15, no. 2, pp. 395-403, 2010.

[48] HANNUKAINEN, A., JUNTUNEN, M., STENBERG, R., Computations with finite element methods for the brinkman problem, Computational Geosciences, vol. 15, no. 1, pp. 155-166, 2011.

[49] WITHERSPOON, P. A., WANG, J. S. Y., IWAI, K., GALE, J. E., Validity of cubic law for fluid flow in a deformable rock fracture, Water Resources Research, vol. 16, no. 6, pp. 1016-1024, 1980.

[50] COMPANY, B. P., Bp statistical review of world energy june 2013, 2013. 
Appendices

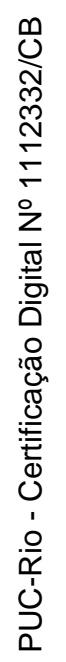




\section{A}

\section{FEniCS Algorithms}

The present algorithms make use of Python 2.7 version programming language and FEniCS 1.2 version.

\section{A.1}

\section{Poisson Equation}

The code listing below compromises a demo present at The FEniCS Project website [42]. This demo solves the Poisson equation with Dirichlet boundary conditions.

The Poisson equation for a domain $\Omega \subset \mathbb{R}^{2}$ with boundary $\Gamma \subset \mathbb{R}^{1}$ reads

$$
-\nabla^{2} u=f \quad \text { in } \Omega .
$$

where $u=u(x, y), f$ is a constant and the boundary conditions defined as:

$$
\begin{aligned}
\nabla u \cdot n & =g \quad \text { on } \Gamma_{N}, \\
u & =u_{0} \quad \text { on } \Gamma_{D} .
\end{aligned}
$$

where $g$ and $u_{0}$ are constants and $n$ denotes the outward directed boundary normal. A variational form of Poisson equation reads as find $u \in V$ such that:

$$
a(u, v)=L(v), \quad \forall v \in V
$$

where $V$ is a suitable function space with $a(u, v)$ and $L(v)$ defined as:

$$
\begin{aligned}
a(u, v) & =\int_{\Omega} \nabla u \cdot \nabla v \mathrm{dx}, \\
L(v) & =\int_{\Omega} f v \mathrm{dx}+\int_{\Gamma_{\mathrm{N}}} \mathrm{g} \mathrm{vds} .
\end{aligned}
$$

The expressions $a(u, v)$ and $L(v)$ are respectively the bilinear and the linear variational forms. It is assumed that all functions in $V$ satisfy the Dirichlet boundary conditions, $u=u_{0}$ on $\Gamma_{D}$. 
Listing A.1: Poisson equation with Dirichlet boundary conditions solved using the Finite Element Method

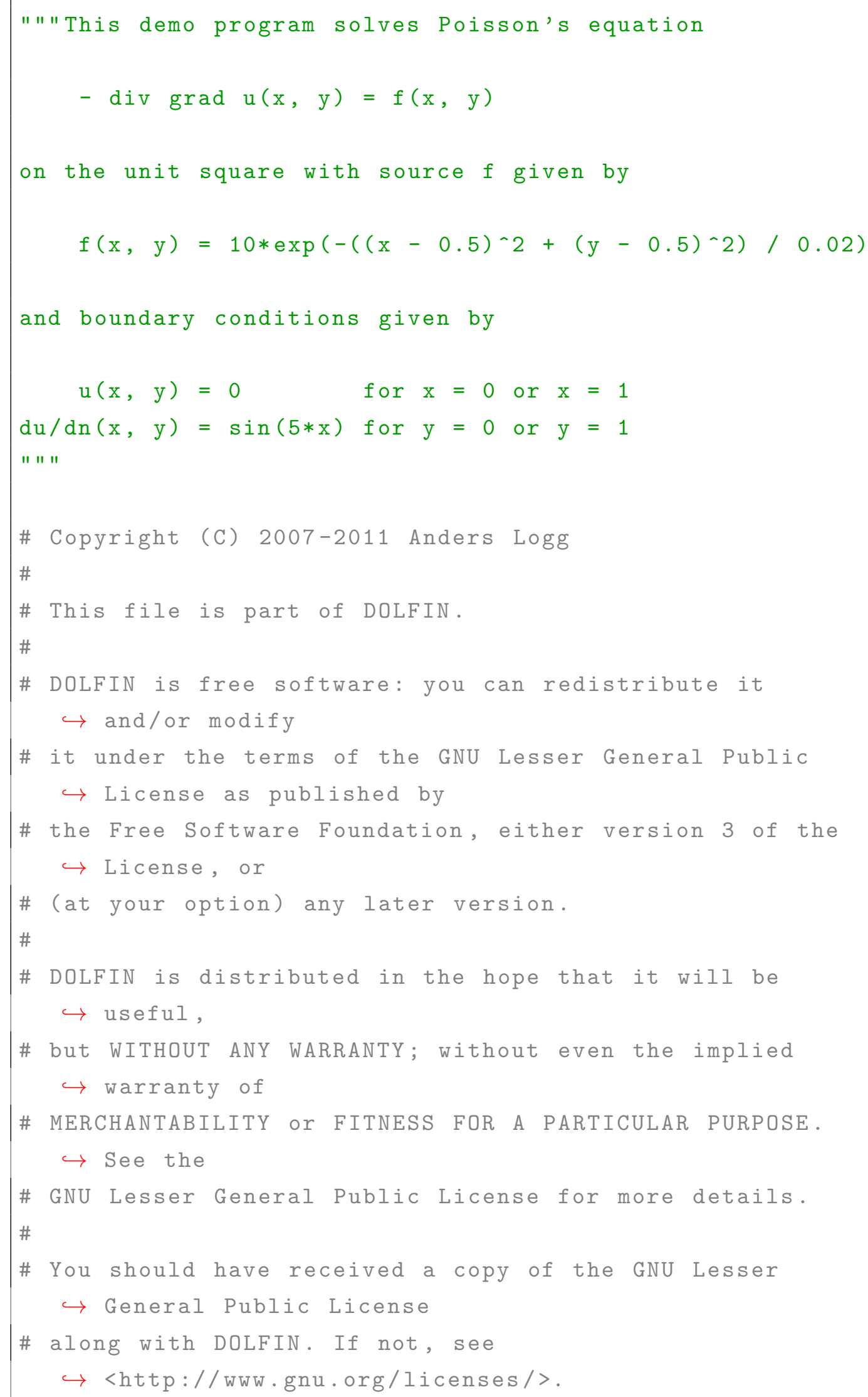




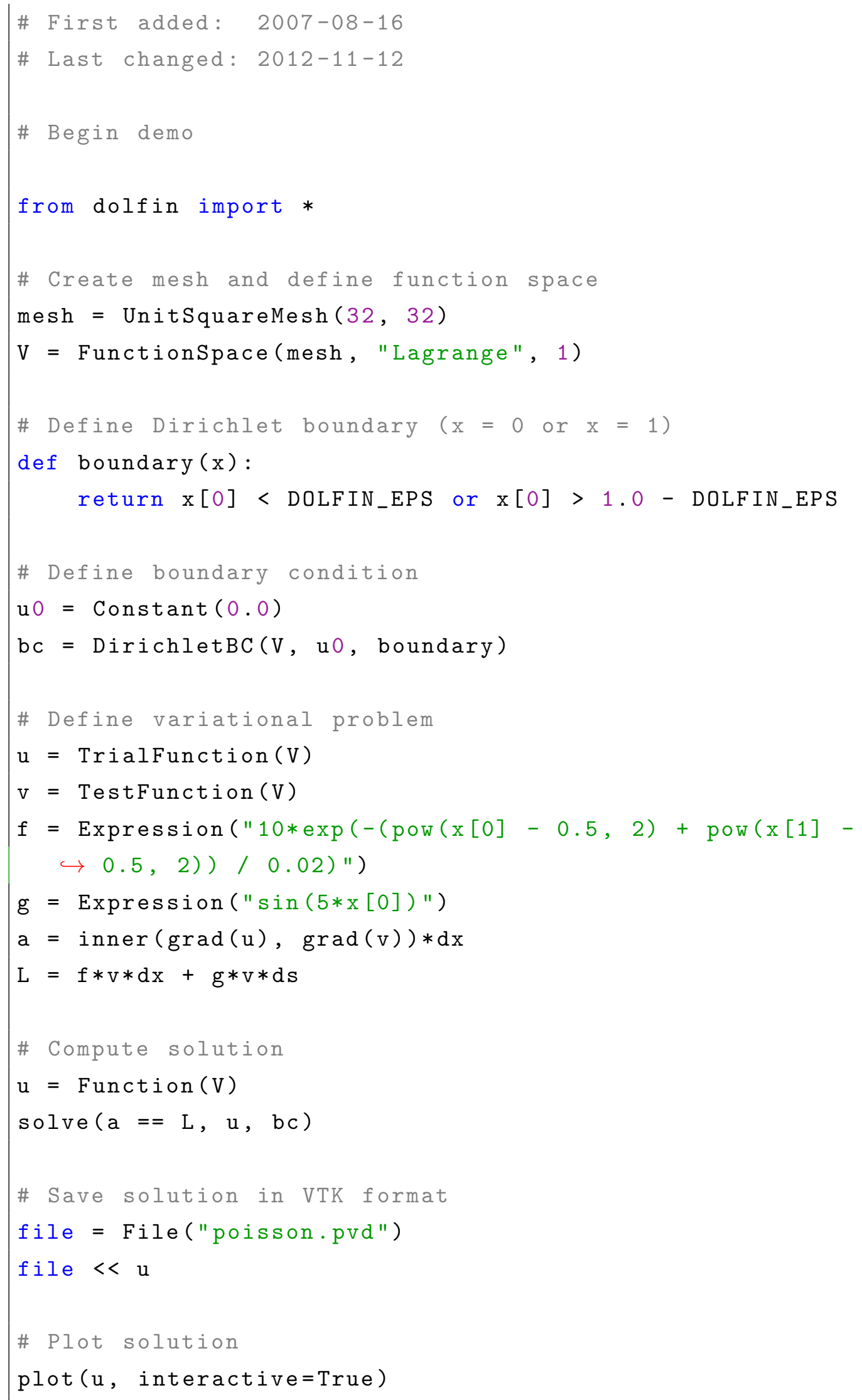




\section{A. 2}

\section{Darcy Equation}

Consider Darcy's flow equation eq. (2-15) described at section 2.3.3 defined for a homogeneous and isotropic medium of permeability $k$ and fluid viscosity $\mu$ :

$$
\begin{aligned}
\mathbf{u}=-\frac{k}{\mu} \nabla p & \text { in } \Omega, \\
\nabla \cdot \mathbf{u}=-f & \text { in } \Omega,
\end{aligned}
$$

with boundary conditions defined as

$$
\begin{aligned}
& \mathbf{u} \cdot n=u_{0} \quad \text { on } \Gamma_{D}, \\
& p=p_{\text {in }} \text { on } \Gamma_{p_{\text {in }}}, \\
& p=p_{\text {out }} \text { on } \Gamma_{p_{\text {out }}} \text {. }
\end{aligned}
$$

where $\Gamma_{p_{\text {in }}}$ and $\Gamma_{p_{\text {out }}} \in \Gamma$ are the boundaries where $p_{\text {in }}$ is the specified entry pressure and $p_{\text {out }}$ the is specified out pressure and $u_{0}$ the velocity pointing out of the Dirichlet boundary $\Gamma_{D}$.

A variational form of the eq. (A-5) reads as find $(\mathbf{u}, p) \in \mathbf{V} \times Q$ such that:

$$
a_{D}(\mathbf{u}, \mathbf{v})+b(\mathbf{v}, p)+b(\mathbf{u}, q)=L(\mathbf{v}) \quad \forall(\mathbf{v}, q) \in \mathbf{V} \times Q,
$$

where the bilinear and linear variational forms $a_{D}, b$ and $L$ are defined as

$$
\begin{aligned}
a_{D}(\mathbf{u}, \mathbf{v}) & =\int_{\Omega} \frac{\mu}{k} \mathbf{u} \cdot \mathbf{v} d x \\
b(\mathbf{v}, p) & =-\int_{\Omega} \nabla \cdot \mathbf{v} p d x \\
L(\mathbf{v}) & =\int_{\Omega} \mathbf{f} \cdot \mathbf{v} d x-\int_{\partial \Omega} g \mathbf{v} \cdot \mathbf{n} d s
\end{aligned}
$$


Listing A.2: Darcy equation with Dirichlet and pressure boundary conditions solved using the Finite Element Method

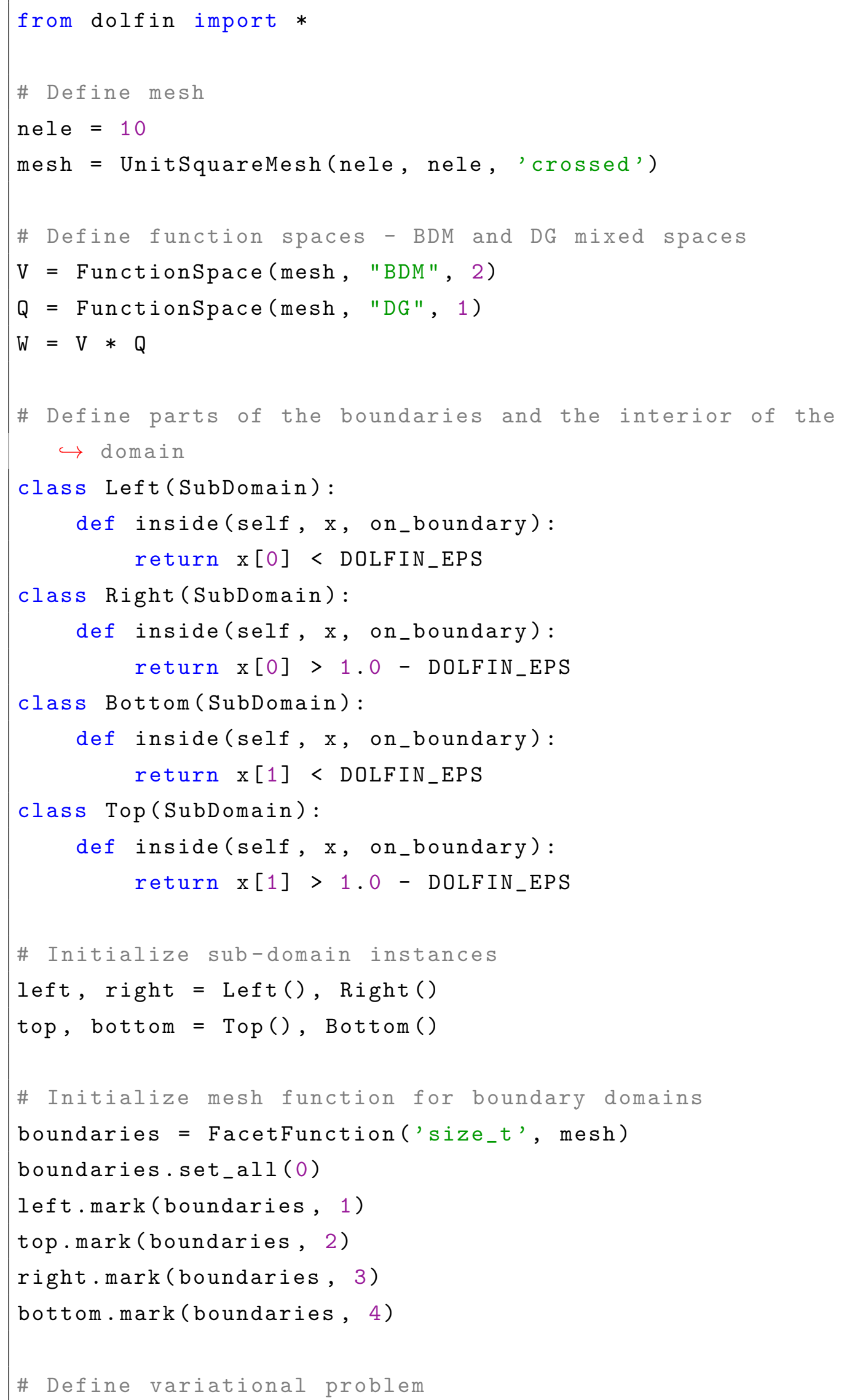




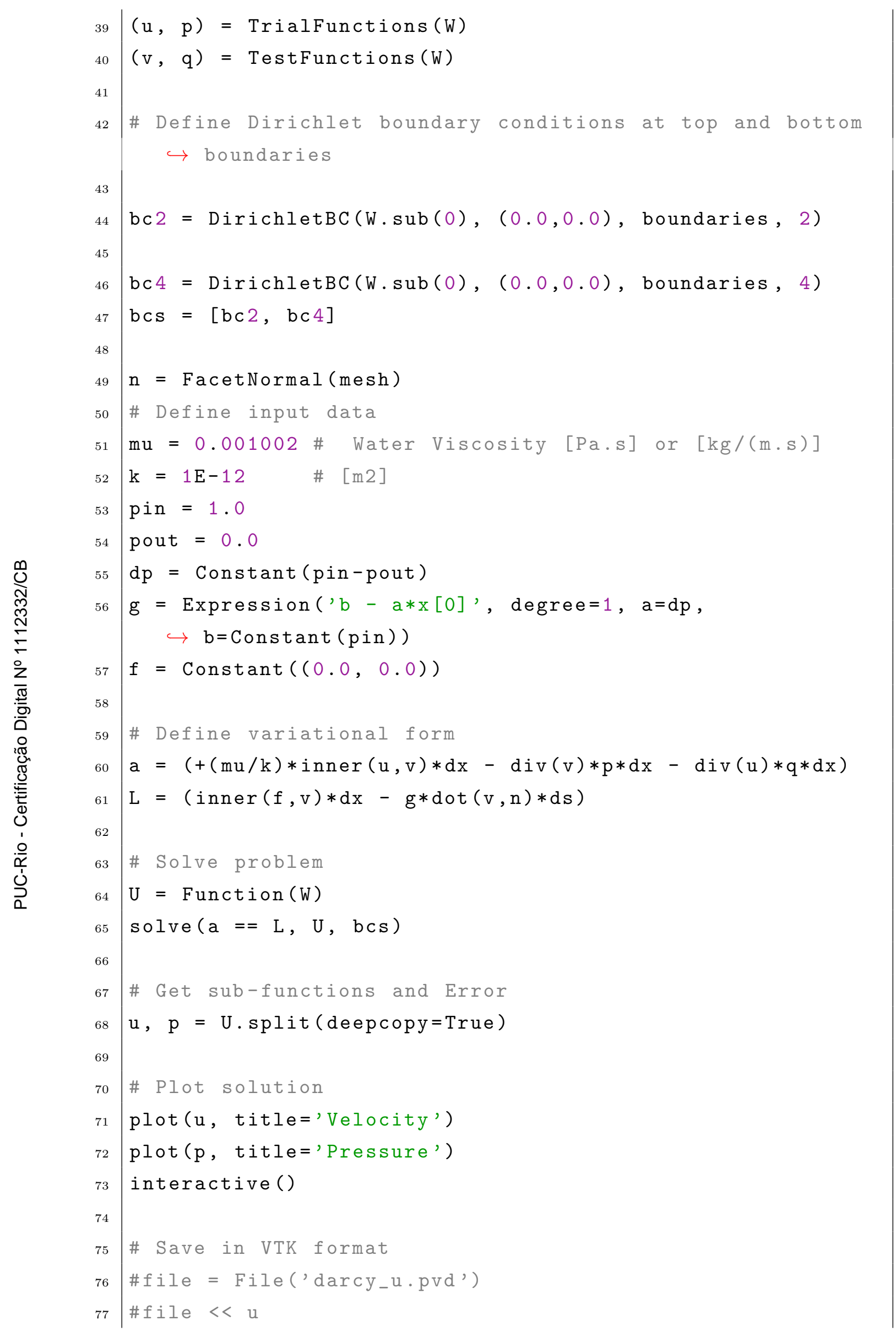


\begin{tabular}{l|l|ll}
78 & $\# f i l e$ & $=F i l e($ \\
79 & $\# f i l e$ & \#
\end{tabular} 


\section{A.3}

\section{Stokes Equation}

Consider Stokes's flow equation eq. (2-13) described at section 2.3.2 for a fluid with viscosity $\mu$ and under steady laminar flow:

$$
\begin{aligned}
\rho \mathbf{g}+\mu \nabla^{2} \mathbf{u}=\nabla p & \text { in } \Omega, \\
\nabla \cdot \mathbf{u}=-f & \text { in } \Omega,
\end{aligned}
$$

with boundary conditions defined as

$$
\begin{aligned}
\mathbf{u} \cdot n & =u_{0} \quad \text { on } \Gamma_{D}, \\
p & =p_{\text {in }} \quad \text { on } \Gamma_{p_{\text {in }}}, \\
p & =p_{\text {out }} \text { on } \Gamma_{p_{\text {out }}} .
\end{aligned}
$$

where $\Gamma_{p_{\text {in }}}$ and $\Gamma_{p_{\text {out }}} \in \Gamma$ are the boundaries where $p_{\text {in }}$ the entry pressure and $p_{\text {out }}$ the out pressure are specified and $u_{0}$ the velocity pointing out of the Dirichlet boundary $\Gamma_{D}$.

A variational form of the $(\mathrm{A}-9)$ reads as find $(\mathbf{u}, p) \in \mathbf{V} \times Q$ such that:

$$
a_{S}(\mathbf{u}, \mathbf{v},)+b(\mathbf{v}, p)+b(\mathbf{u}, q)=L(\mathbf{v}) \quad \forall(\mathbf{v}, q) \in \mathbf{V} \times Q,
$$

where the bilinear and linear variational forms $a_{S}, b$ and $L$ are defined as

$$
\begin{aligned}
a_{S}(\mathbf{u}, \mathbf{v}) & =\int_{\Omega} \nabla \mathbf{u}: \nabla \mathbf{v} d x \\
b(\mathbf{v}, p) & =-\int_{\Omega} \nabla \cdot \mathbf{v} p d x \\
L(\mathbf{v}) & =\int_{\Omega} \mathbf{f} \cdot \mathbf{v} d x+\int_{\Gamma} g \mathbf{v} \cdot \mathbf{n} d s .
\end{aligned}
$$


Listing A.3: Stokes equation with Dirichlet and pressure boundary conditions solved using the Finite Element Method

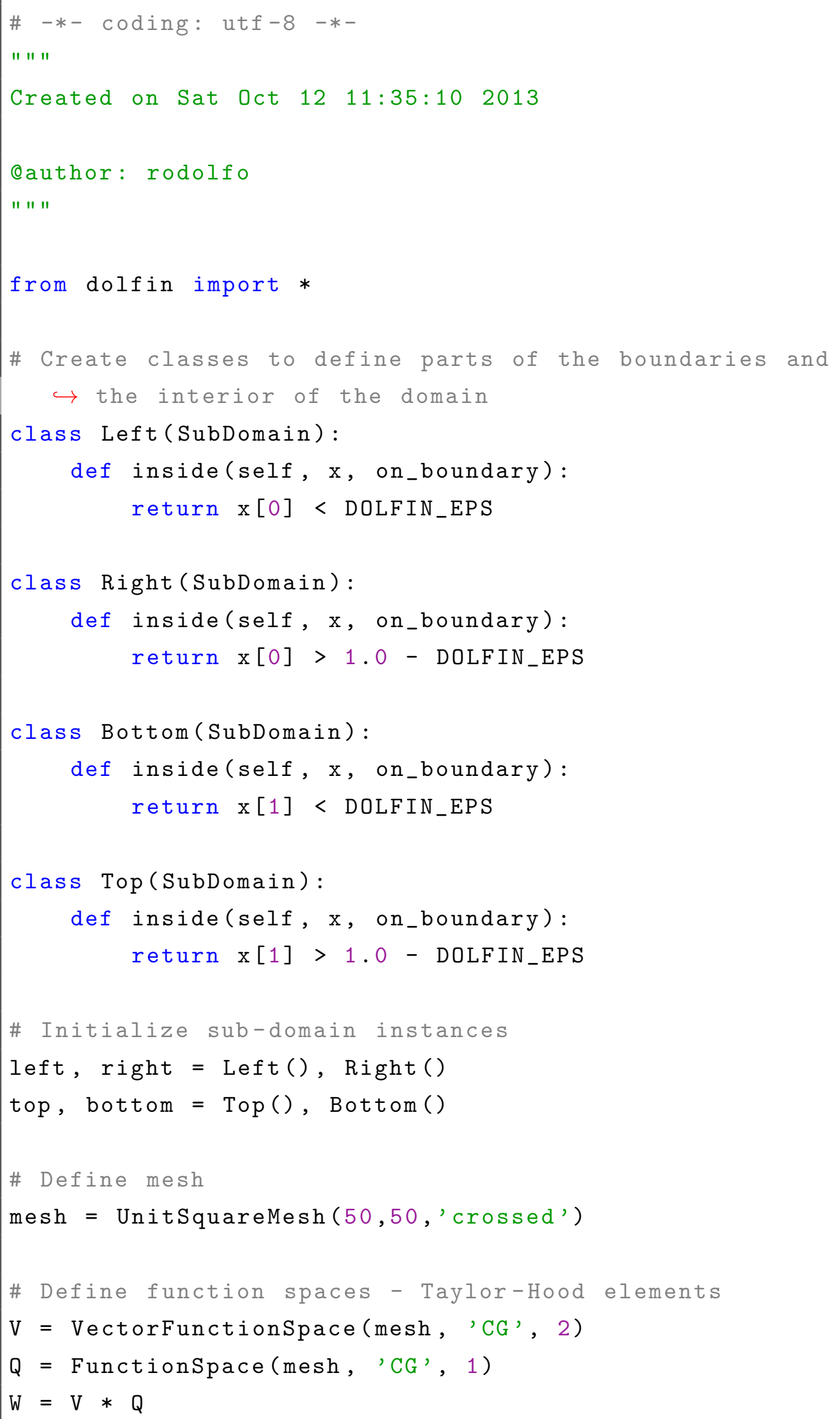




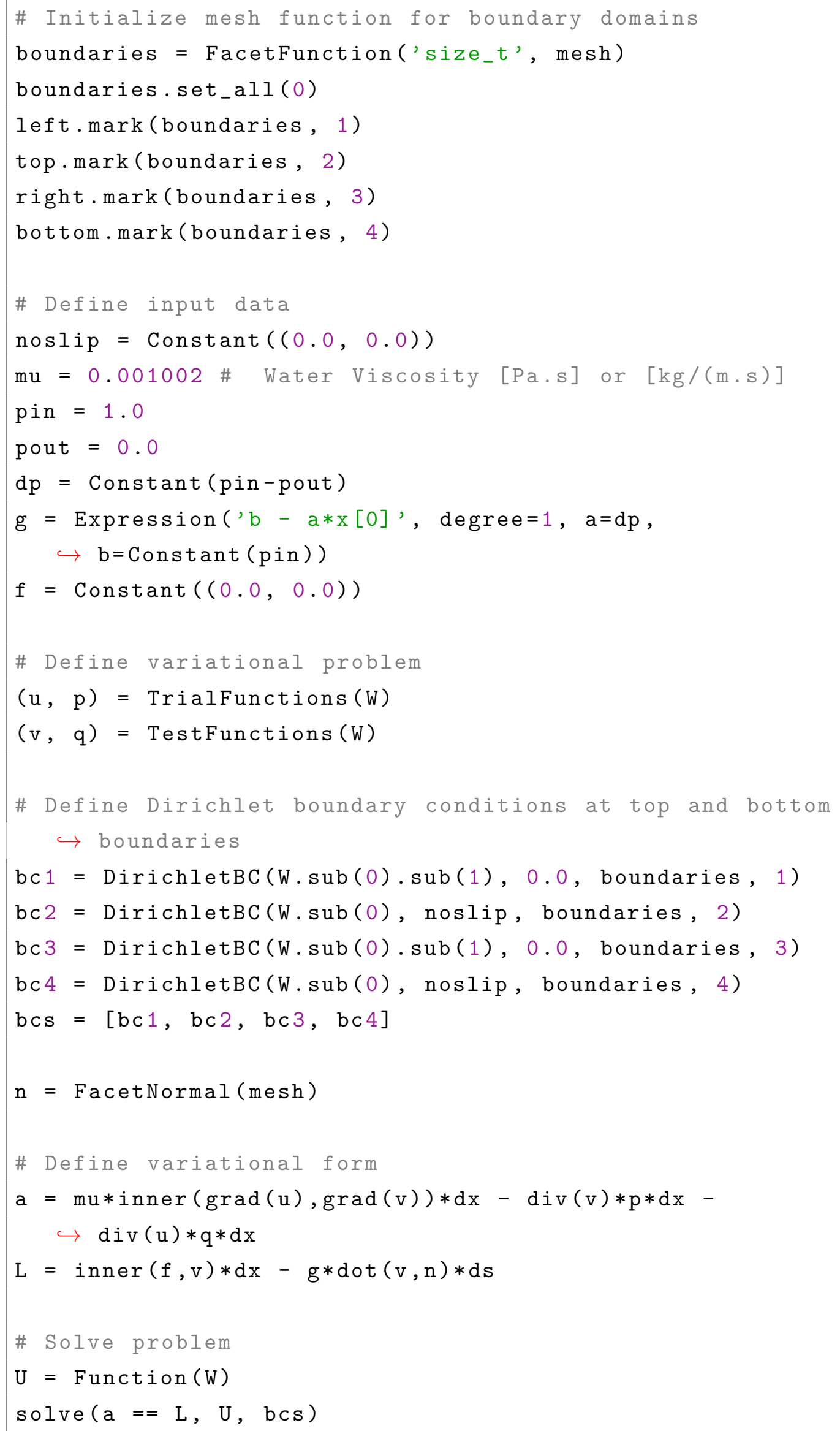




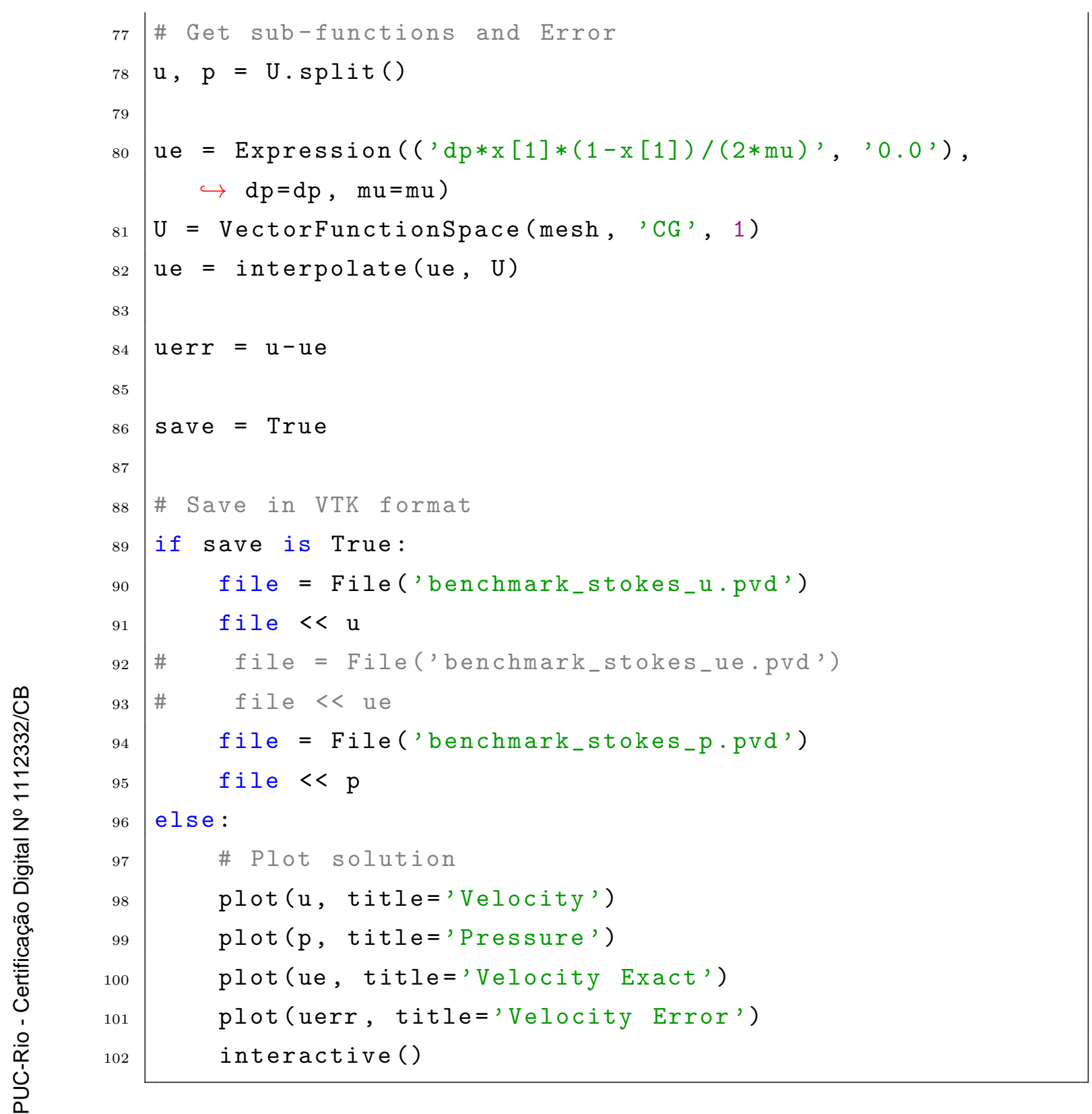




\section{A.4}

\section{Brinkman Equation}

Consider Brinkman's flow equation eq. (2-16) described at section 2.3.4 for a homogeneous and isotropic medium of permeability $k$ and fluid viscosity $\mu$ :

$$
\begin{aligned}
-\mu^{*} \nabla^{2} \mathbf{u}+\nabla p+\mu \mathbf{k}^{-1} \mathbf{u}=\mathbf{f} & & \text { in } \Omega, \\
\nabla \cdot \mathbf{u}=g & & \text { in } \Omega,
\end{aligned}
$$

with boundary conditions defined as

$$
\begin{aligned}
\mathbf{u} \cdot n & =u_{0} \quad \text { on } \Gamma_{D}, \\
p & =p_{\text {in }} \quad \text { on } \Gamma_{p_{\text {in }}}, \\
p & =p_{\text {out }} \text { on } \Gamma_{p_{\text {out }}} .
\end{aligned}
$$

where $\Gamma_{p_{\text {in }}}$ and $\Gamma_{p_{\text {out }}} \in \Gamma$ are the boundaries where $p_{\text {in }}$ the entry pressure and $p_{\text {out }}$ the out pressure are specified and $u_{0}$ the velocity pointing out of the Dirichlet boundary $\Gamma_{D}$.

A variational form of the $(\mathrm{A}-9)$ reads as find $(\mathbf{u}, p) \in \mathbf{V} \times Q$ such that:

$$
a_{B}(\mathbf{u}, \mathbf{v})+b(\mathbf{v}, p)+b(\mathbf{u}, q)=L(\mathbf{v}, q) \quad \forall(\mathbf{v}, q) \in \mathbf{V} \times Q
$$

where the bilinear forms $a_{B}$ and $b$ and the linear form $L$ are defined as

$$
\begin{aligned}
a_{B}(\mathbf{u}, \mathbf{v}) & =\int_{\Omega} \mu \nabla \mathbf{u}: \nabla \mathbf{v} d x+\int_{\Omega} \frac{\mu}{k} \mathbf{u} \cdot \mathbf{v} d x, \\
b(\mathbf{v}, q) & =\int_{\Omega} \nabla \cdot \mathbf{v} q d x \\
L(\mathbf{v}) & =\int_{\Omega} \mathbf{f} \cdot \mathbf{v} d x+\int_{\Gamma} g \mathbf{v} \cdot \mathbf{n} d s .
\end{aligned}
$$


Listing A.4: Brinkman equation with Dirichlet and pressure boundary conditions solved using the Finite Element Method

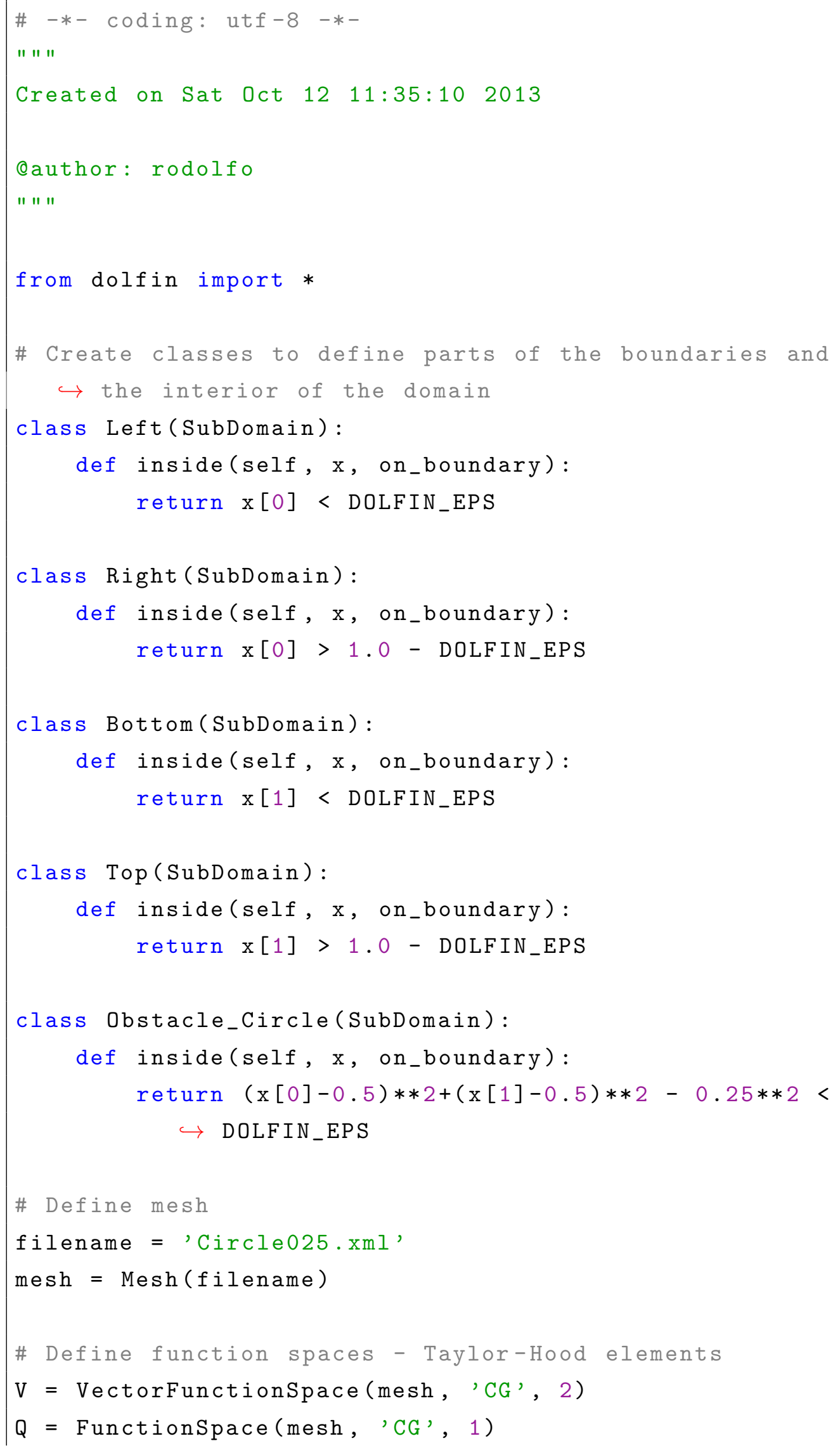




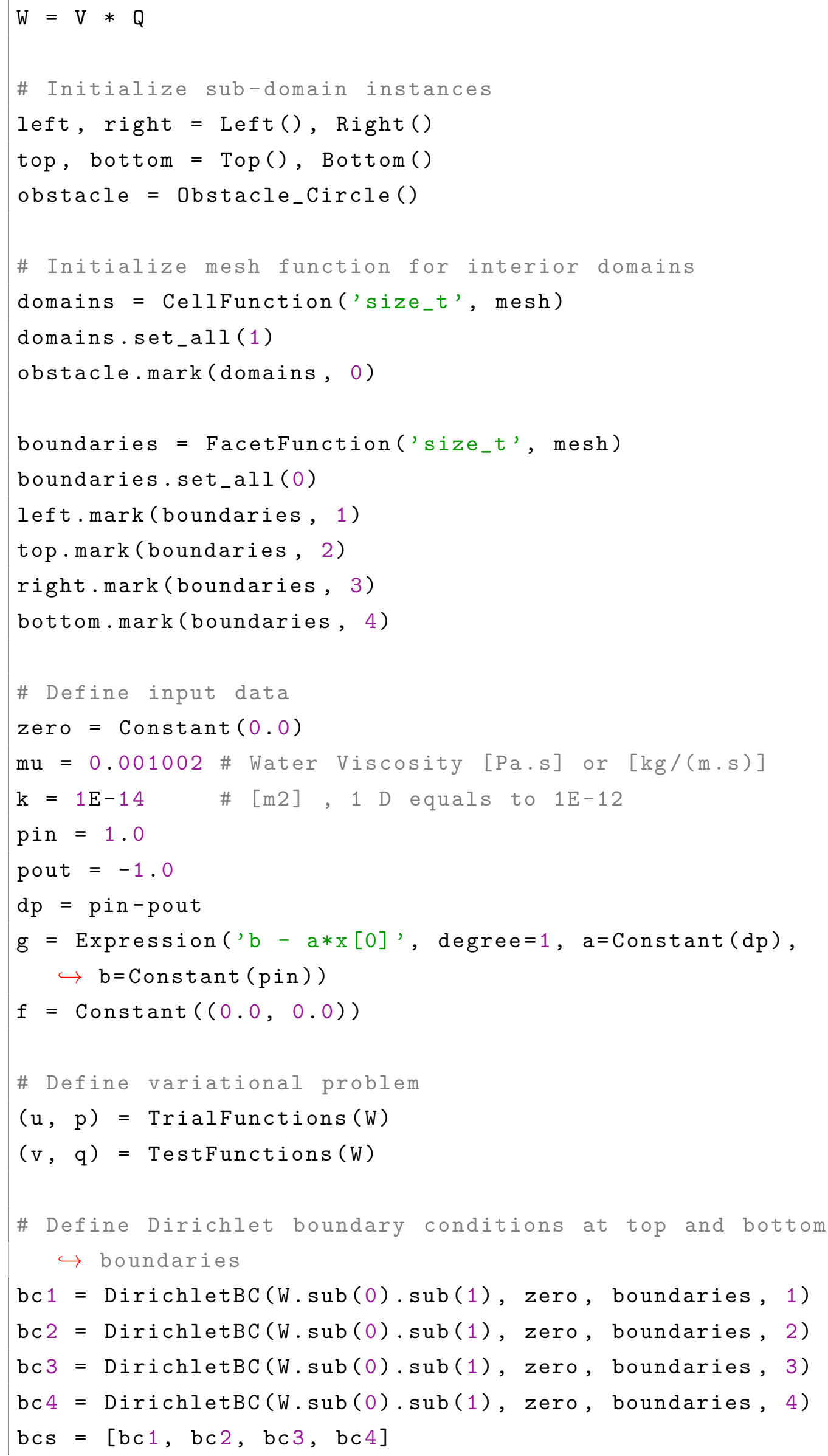


\# Define new measures associated with the domains and

$\hookrightarrow$ boundaries

$\mathrm{dx}=$ Measure(' $\left.\mathrm{dx} \mathrm{x}^{\prime}\right)$ [subdomains $]$

$\mathrm{d} s=$ Measure('ds') [boundaries]

$\mathrm{n}=$ FacetNormal (mesh)

\# Define variational form

$a=(\operatorname{mu} *$ inner $(\operatorname{grad}(u), \operatorname{grad}(v)) * d x(0)$

$+(\mathrm{mu} / \mathrm{k}) *$ inner $(\mathrm{u}, \mathrm{v}) * \mathrm{dx}(1)$

$-\operatorname{div}(\mathrm{v}) * \mathrm{p} * \mathrm{dx}(0)-\operatorname{div}(\mathrm{v}) * \mathrm{p} * \mathrm{dx}(1)$

$-\operatorname{div}(u) * q * d x(0)-\operatorname{div}(u) * q * d x(1))$

$\mathrm{L}=(\operatorname{inner}(\mathrm{f}, \mathrm{v}) * \mathrm{dx}(0)+\operatorname{inner}(\mathrm{f}, \mathrm{v}) * \mathrm{dx}(1)$

$-\mathrm{g} * \operatorname{dot}(\mathrm{v}, \mathrm{n}) * \operatorname{ds}(1)-\mathrm{g} * \operatorname{dot}(\mathrm{v}, \mathrm{n}) * \operatorname{ds}(3))$

\# Solve problem

$\mathrm{U}=$ Function $(W)$

solve $(a==L, U, b c s)$

\# Get sub-functions

$\mathrm{u}, \mathrm{p}=\mathrm{U} \cdot \operatorname{split}()$

save $=$ False

\# Save in VTK format

if save is False: \# Plot solution

plot (u, title='Velocity')

plot (p, title='Pressure')

interactive()

else: \# Save in VTK format

file $=$ File (fullpath+filename+' _u.pvd')

file $<u$

file = File(fullpath+filename+' _p.pvd')

file $<p$ 